\title{
Review Article \\ Particle Production in Strong Electromagnetic Fields in Relativistic Heavy-Ion Collisions
}

\author{
Kirill Tuchin \\ Department of Physics and Astronomy, Iowa State University, Ames, IA 50011, USA \\ Correspondence should be addressed to Kirill Tuchin; tuchink@gmail.com
}

Received 31 December 2012; Accepted 11 April 2013

Academic Editor: Jan E. Alam

Copyright ( 2013 Kirill Tuchin. This is an open access article distributed under the Creative Commons Attribution License, which permits unrestricted use, distribution, and reproduction in any medium, provided the original work is properly cited.

I review the origin and properties of electromagnetic fields produced in heavy-ion collisions. The field strength immediately after a collision is proportional to the collision energy and reaches $\sim m_{\pi}^{2}$ at RHIC and $\sim 10 m_{\pi}^{2}$ at LHC. I demonstrate by explicit analytical calculation that after dropping by about one-two orders of magnitude during the first $\mathrm{fm} / \mathrm{c}$ of plasma expansion, it freezes out and lasts for as long as quark-gluon plasma lives as a consequence of finite electrical conductivity of the plasma. Magnetic field breaks spherical symmetry in the direction perpendicular to the reaction plane, and therefore all kinetic coefficients are anisotropic. I examine viscosity of QGP and show that magnetic field induces azimuthal anisotropy on plasma flow even in spherically symmetric geometry. Very strong electromagnetic field has an important impact on particle production. I discuss the problem of energy loss and polarization of fast fermions due to synchrotron radiation, consider photon decay induced by magnetic field, elucidate $J / \psi$ dissociation via Lorentz ionization mechanism, and examine electromagnetic radiation by plasma. I conclude that all processes in QGP are affected by strong electromagnetic field and call for experimental investigation.

\section{Origin and Properties of Electromagnetic Field}

1.1. Origin of Magnetic Field. We can understand the origin of magnetic field in heavy-ion collisions by considering collision of two ions of radius $R$ with electric charge $Z e(e$ is the magnitude of electron charge) at impact parameter $\mathbf{b}$. According to the Biot and Savart law they create magnetic field that in the center-of-mass frame has magnitude

$$
B \sim \gamma Z e \frac{b}{R^{3}}
$$

and points in the direction perpendicular to the reaction plane (span by the momenta of ions). Here $\gamma=\sqrt{s_{N N}} / 2 m_{N}$ is the Lorentz factor. At RHIC heavy ions are collided at $200 \mathrm{GeV}$ per nucleon, hence $\gamma=100$. Using $Z=79$ for gold and $b \sim R_{A} \approx 7 \mathrm{fm}$ we estimate $e B \approx m_{\pi}^{2} \sim 10^{18} \mathrm{G}$. To appreciate how strong is this field, compare it with the following numbers: the strongest magnetic field created on earth in a form of electromagnetic shock wave is $\sim 10^{7} \mathrm{G}[1]$, and magnetic field of a neutron star is estimated to be $10^{10}$ $10^{13} \mathrm{G}$, that of a magnetar up to $10^{15} \mathrm{G}[2]$. It is perhaps the strongest magnetic field that has ever existed in nature.

It has been known for a long time that classical electrodynamics breaks down at the critical (Schwinger) field strength $F=m_{e}^{2} / e$ [3]. In cgs units the corresponding magnetic field is $10^{13} \mathrm{G}$. Because $m_{\pi} / m_{e}=280$, electromagnetic fields created at RHIC and LHC are well above the critical value. This offers a unique opportunity to study the super-strong electromagnetic fields in laboratory. The main challenge is to identify experimental observables that are sensitive to such fields. The problem is that nearly all observables studied in heavy-ion collisions are strongly affected both by the strong color forces acting in quark-gluon plasma (QGP) and by electromagnetic fields often producing qualitatively similar effects. An outstanding experimental problem thus is to separate the two effects. In Sections 2-7 I examine several processes strongly affected by intense magnetic fields and discuss their phenomenological significance. But first, in this section, let me derive a quantitative estimate of electromagnetic field. 
Throughout this paper, the heavy-ion collision axis is denoted by $z$. Average magnetic field then points in the $y$ direction; see Figures 1 and 7. Plane $x z$ is the reaction plane, and $b$ is the impact parameter.

\subsection{Magnetic Field in Vacuum}

1.2.1. Time Dependence. To obtain a quantitative estimate of magnetic field we need to take into account a realistic distribution of protons in a nucleus. This has been first done in [5] (in the case of high-energy $p p$ collisions, magnetic field was first estimated in [9] who also pointed out a possibility of formation of $W$-condensate $[9,10])$. Magnetic field at point $\mathbf{r}$ created by two heavy ions moving in the positive or negative $z$-direction can be calculated using the Liénard-Wiechert potentials as follows:

$$
\begin{aligned}
& e \mathbf{E}(t, \mathbf{r})=\alpha_{\mathrm{em}} \sum_{a} \frac{\left(1-v_{a}^{2}\right) \mathbf{R}_{a}}{R_{a}^{3}\left[1-\left(\mathbf{R}_{a} \times \mathbf{v}_{a}\right)^{2} / R_{a}^{2}\right]^{3 / 2}}, \\
& e \mathbf{B}(t, \mathbf{r})=\alpha_{\mathrm{em}} \sum_{a} \frac{\left(1-v_{a}^{2}\right)\left(\mathbf{v}_{a} \times \mathbf{R}_{a}\right)}{R_{a}^{3}\left[1-\left(\mathbf{R}_{a} \times \mathbf{v}_{a}\right)^{2} / R_{a}^{2}\right]^{3 / 2}},
\end{aligned}
$$

with $\mathbf{R}_{a}=\mathbf{r}-\mathbf{r}_{a}(t)$, where sums run over all $Z$ protons in each nucleus, their positions and velocities being $\mathbf{r}_{a}$ and $\mathbf{v}_{a}$. The magnitude of velocity $v_{a}$ is determined by the collision energy $\sqrt{s_{N N}}$ and the proton mass $m_{p}, v_{a}^{2}=1-\left(2 m_{p} / \sqrt{s_{N N}}\right)^{2}$. These formulas are derived in the eikonal approximation, assuming that protons travel on straight lines before and after the scattering. This is a good approximation, since baryon stopping is a small effect at high energies. Positions of protons in heavy ions can be determined by one of the standard models of the nuclear charge density $\rho\left(\mathbf{r}_{a}\right)$. Reference [5] employed the "hard sphere" model, while [4] used a bit more realistic Woods-Saxon distribution.

Numerical integration in (3) including small contribution from baryon stopping yields for magnetic field the result shown in Figure 2 as a function of the proper time $\tau=\left(t^{2}-\right.$ $\left.z^{2}\right)^{1 / 2}$. Evidently, magnetic field rapidly decreases as a power of time, so that after first $3 \mathrm{fm}$ it drops by more than three orders of magnitude.

\subsubsection{Event-by-Event Fluctuations in Proton Positions.} Nuclear charge density $\rho$ provides only event-averaged distribution of protons. The actual distribution in a given event is different form $\rho$ implying that in a single event there is not only magnetic field along the $y$-direction, but also other components of electric and magnetic fields. This leads to event-by-event fluctuations of electromagnetic field [4]. Shown in Figure 3 are electric and magnetic field components at $t=0$ at the origin (denoted by a black dot in Figure 1) in AuAu collisions at $\sqrt{s_{N N}}=200 \mathrm{GeV}$.

Figure 3 clearly shows that although on average the only nonvanishing component of the field is $B_{y}$, which is also clear

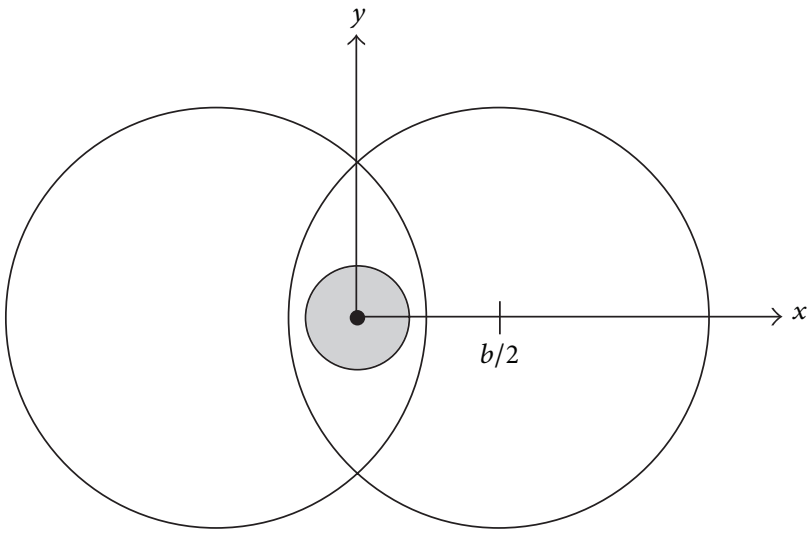

FIGURE 1: Heavy-ion collision geometry as seen along the collision axis $z$. Adapted from [4].

from the symmetry considerations, other components are finite in each event and are of the same order of magnitude

$$
\left\langle\left|B_{x}\right|\right\rangle \approx\left\langle\left|E_{x}\right|\right\rangle \approx\left\langle\left|E_{y}\right|\right\rangle
$$

To appreciate the magnitude of electric field produced in heavy-ion collisions note that $E \sim m_{\pi}^{2}=10^{21} \mathrm{~V} / \mathrm{cm}$. The corresponding intensity is $10^{39} \mathrm{~W} / \mathrm{cm}^{2}$ which is instructive to compare with the power generated by the most powerful state-of-the-art lasers: $10^{23} \mathrm{~W} / \mathrm{cm}^{2}$.

Electromagnetic fields created in heavy-ion collisions were also examined in more elaborated approaches in [11-13]. They yielded qualitatively similar results on electromagnetic field strength and its relaxation time.

\subsection{Magnetic Field in Quark-Gluon Plasma}

1.3.1. Liénard-Wiechert Potentials in Static Medium. In the previous section, I discussed electromagnetic field in vacuum. A more realistic estimate must include medium effects. Indeed, the state-of-the-art phenomenology of quark-gluon plasma (QGP) indicates that strongly interacting medium is formed at as early as $0.5 \mathrm{fm} / \mathrm{c}$. Even before this time, strongly interacting medium exists in a form of Glasma $[14,15]$. Therefore, a calculation of magnetic field must involve response of medium determined by its electrical conductivity. It has been found in the lattice calculations that the gluon contribution to electrical conductivity of static quark-gluon plasma is [16]

$$
\sigma=(5.8 \pm 2.9) \frac{T}{T_{c}} \mathrm{MeV}
$$

where $T$ is plasma temperature and $T_{c}$ it critical temperature. This agrees with [17] but is at odds with an earlier calculation [18]. It is not clear whether (5) adequately describes the electromagnetic response of realistic quarkgluon plasma because it neglects quark contribution and 


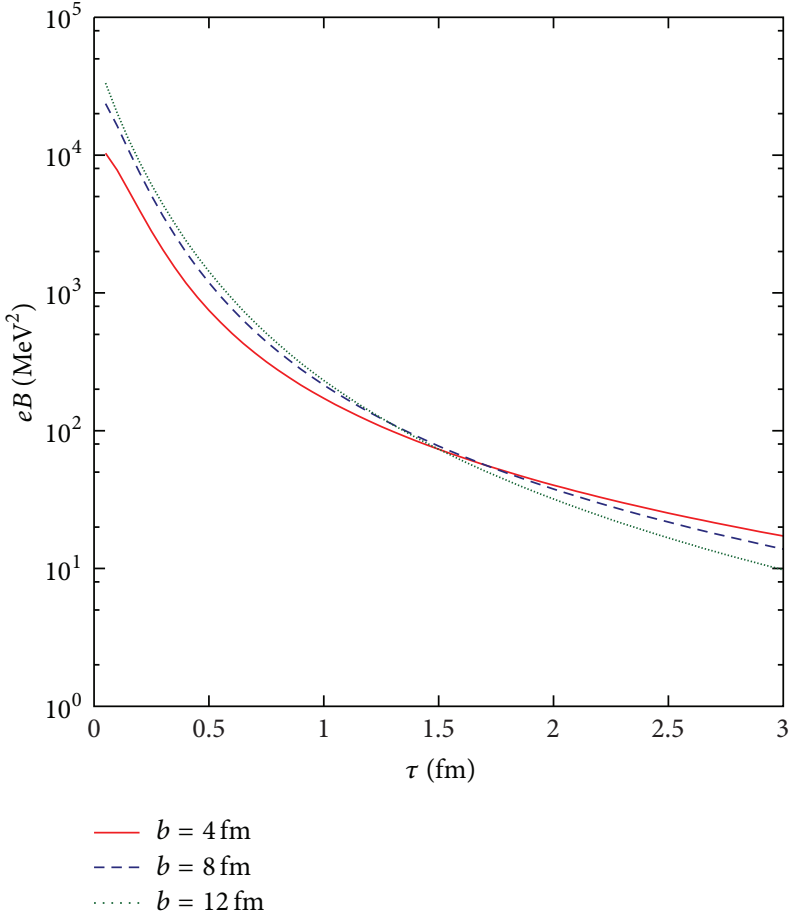

(a)

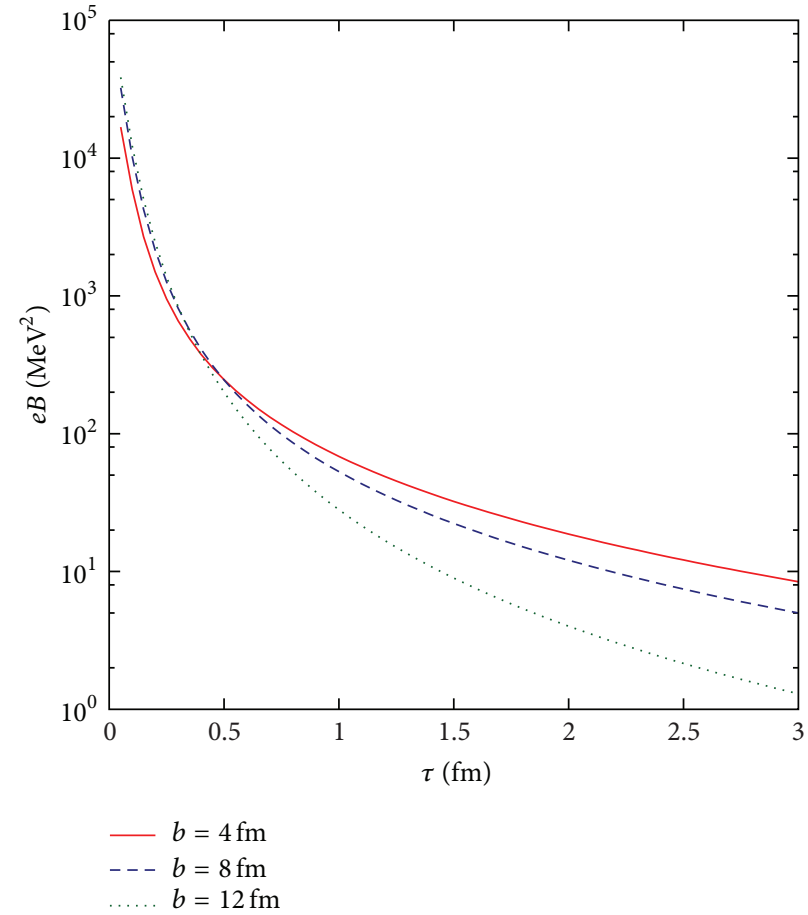

(b)

Figure 2: Magnetic field $\mathbf{B}=B \widehat{\mathbf{y}}$ (multiplied by $e$ ) at the origin $\mathbf{r}=0$ produced in collision of two gold ions at beam energies $(\mathrm{a}) \sqrt{s_{N N}}=$ $62 \mathrm{GeV}$ and (b) $\sqrt{s_{N N}}=200 \mathrm{GeV}$. Adapted from [5]. Note that $e B$ is the same in Gauss and Lorentz-Heaviside units in contrast to $B$.

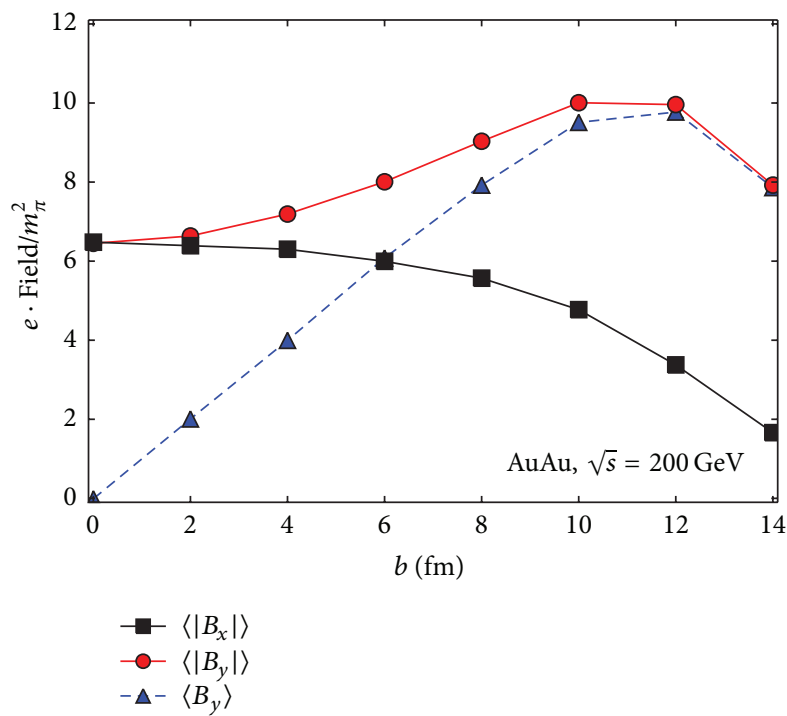

(a)

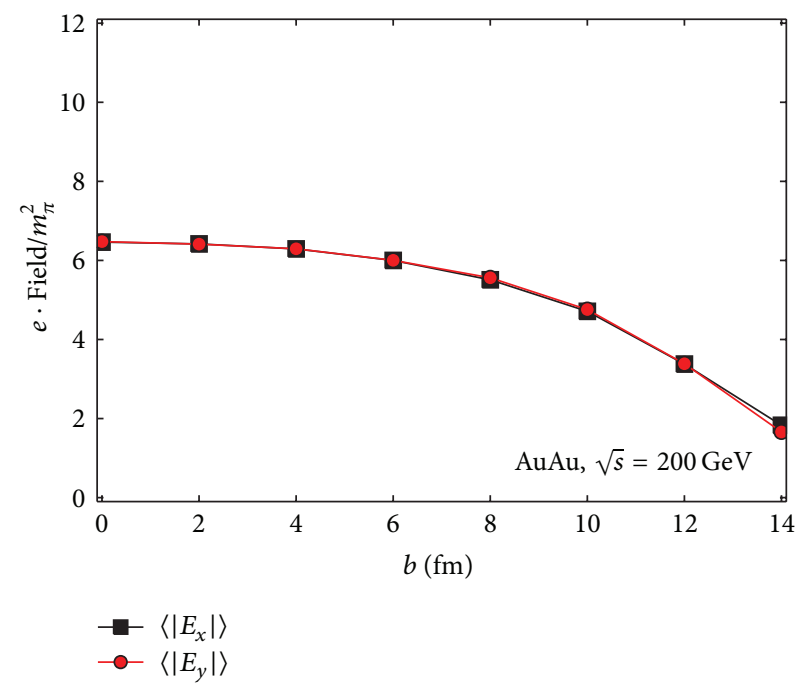

(b)

Figure 3: The mean absolute value of (a) magnetic field and (b) electric field at $t=0$ and $\mathbf{r}=0$ as a function of impact parameter $b$ for AuAu collision at $\sqrt{s_{N N}}=200 \mathrm{GeV}$.

assumes that medium is static. Theoretical calculations are of little help at the temperatures of interest, since the perturbation theory is not applicable. In absence of a sensible alternative I will use (5) as a best estimate of electrical conductivity. If medium is static then $T$ is constant as a function of time $t$. The static case is considered in this section, while in the next section I consider expanding medium.

In medium, magnetic field created by a charge $e$ moving in $z$-direction with velocity $v$ is a solution of the following 
equations:

$$
\begin{gathered}
\nabla \cdot \mathbf{B}=0, \quad \nabla \times \mathbf{E}=-\frac{\partial \mathbf{B}}{\partial t}, \\
\nabla \cdot \mathbf{E}=e \delta(z-v t) \delta(\mathbf{b}), \\
\nabla \times \mathbf{B}=\frac{\partial \mathbf{E}}{\partial t}+\sigma \mathbf{E}+e v \widehat{\mathbf{z}} \delta(z-v t) \delta(\mathbf{b}),
\end{gathered}
$$

where we used the Ohm's law $\mathbf{j}=\sigma \mathbf{E}$ to describe currents induced in the medium. Position of the observation point is specified by the longitudinal and transverse coordinates $z$ and $\mathbf{b}, \mathbf{r}=z \widehat{\mathbf{z}}+\mathbf{b}$. Taking curl of the second equation in (7) and substituting (6) we get

$$
\nabla^{2} \mathbf{B}=\frac{\partial^{2} \mathbf{B}}{\partial t^{2}}+\sigma \frac{\partial \mathbf{B}}{\partial t}-e v \nabla \times[\widehat{\mathbf{z}} \delta(z-v t) \delta(\mathbf{b})] .
$$

The particular solution reads

$$
\begin{gathered}
\mathbf{B}(z, \mathbf{b}, t)=\int_{0}^{t} d t^{\prime} \int_{-\infty}^{\infty} d z \int d^{2} \mathbf{b} G\left(z-z^{\prime}, \mathbf{b}-\mathbf{b}^{\prime}, t-t^{\prime}\right) e v \\
\times \nabla^{\prime}\left[\widehat{\mathbf{z}} \delta\left(z^{\prime}-v t^{\prime}\right) \delta\left(\mathbf{b}^{\prime}\right)\right],
\end{gathered}
$$

where Green's function $G\left(z-z^{\prime}, \mathbf{b}-\mathbf{b}^{\prime}, t-t^{\prime}\right)$ satisfies the following equation:

$$
\nabla^{2} G-\frac{\partial^{2} G}{\partial t^{2}}-\sigma \frac{\partial G}{\partial t}=-\delta\left(z-z^{\prime}\right) \delta\left(\mathbf{b}-\mathbf{b}^{\prime}\right) \delta\left(t-t^{\prime}\right)
$$

which is solved by

$$
\begin{aligned}
& G(z-\left.z^{\prime}, \mathbf{b}-\mathbf{b}^{\prime}, t-t^{\prime}\right) \\
&= \int \frac{d^{2} k_{\perp}}{(2 \pi)^{2}} e^{i\left(\mathbf{b}-\mathbf{b}^{\prime}\right) \cdot \mathbf{k}_{\perp}} \int_{-\infty}^{\infty} \frac{d k_{z}}{2 \pi} e^{i k_{z}\left(z-z^{\prime}\right)} \\
& \quad \times \int_{-\infty}^{\infty} \frac{d \omega}{2 \pi} e^{-i \omega\left(t-t^{\prime}\right)} \frac{1}{k_{z}^{2}+k_{\perp}^{2}-\omega^{2}-i \omega \sigma},
\end{aligned}
$$

where $\mathbf{k}=k_{z} \widehat{\mathbf{z}}+\mathbf{k}_{\perp}$. Plugging this into (9) and substituting for the expression in the square brackets in (9) its Fourier image, we obtain

$$
\begin{gathered}
\mathbf{B}(z, \mathbf{b}, t)=2 \pi e v \int \frac{d^{2} k_{\perp}}{(2 \pi)^{2}} e^{i \mathbf{b} \cdot \mathbf{k}_{\perp}} \int_{-\infty}^{\infty} \frac{d k_{z}}{2 \pi} e^{i k_{z} z} \\
\times \int_{-\infty}^{\infty} \frac{d \omega}{2 \pi} e^{-i \omega t} \frac{i \mathbf{k} \times \widehat{\mathbf{z}}}{k_{z}^{2}+k_{\perp}^{2}-\omega^{2}-i \omega \sigma} \\
\times \delta\left(\omega-k_{z} v\right) \\
=e \int \frac{d^{2} k_{\perp}}{(2 \pi)^{2}} e^{i \mathbf{b} \cdot \mathbf{k}_{\perp}} \\
\times \int_{-\infty}^{\infty} \frac{d \omega}{2 \pi} e^{-i \omega t} e^{i \omega z / v} \\
\times \frac{i \mathbf{k}_{\perp} \times \widehat{\mathbf{z}}}{\omega^{2} / v^{2}+k_{\perp}^{2}-\omega^{2}-i \omega \sigma} .
\end{gathered}
$$

We are interested in the $y$-component of the field. Noting that $\left(\mathbf{k}_{\perp} \times \widehat{\mathbf{z}}\right) \cdot \widehat{\mathbf{y}}=-k_{\perp} \cos \phi$, where $\phi$ is the azimuthal angle in the transverse plane, and integrating over $d^{2} k_{\perp}$ we derive

$$
e B_{y}=\frac{\alpha_{\mathrm{em}}}{\pi} \int_{-\infty}^{\infty} s(\omega) K_{1}(s(\omega) b) e^{i \omega(z / v-t)} d \omega,
$$

where $\alpha_{\mathrm{em}}=e^{2} / 4 \pi$, and we introduced notation

$$
s(\omega)=\omega \sqrt{\frac{1}{v^{2}}-\epsilon(\omega)},
$$

where $\epsilon(\omega)$ is the dielectric constant of the plasma with the following frequency dependence:

$$
\epsilon(\omega)=1+\frac{i \sigma}{\omega}
$$

Equation (14) is actually valid for any functional form of $\epsilon(\omega)$ [19], which can be easily verified by using electric displacement $\mathbf{D}$ instead of $\mathbf{E}$ in (7). In this case (16) can be viewed as a low frequency expansion of $\epsilon(\omega)$. Magnetic field in this approximation is quasistatic. Therefore, we could have neglected the second time derivative in (8), and then keeping only the leading powers of $\omega$ we would have derived (14) with $s^{2}=i \omega \sigma$. After integration over $\omega$ this gives (21). Let us take notice of the fact that neglecting the second time derivative in (8) yields diffusion equation for magnetic field in plasma.

It is instructive to compare time dependence of magnetic field created by moving charges in vacuum and in plasma. In vacuum, setting $\sigma=0$ in (13) and integrating first over $\omega$ and then over $\mathbf{k}_{\perp}$ give

$$
e \mathbf{B}=\widehat{\mathbf{y}} \alpha_{\mathrm{em}} \frac{b \gamma}{\left(b^{2}+\gamma^{2}(t-z)^{2}\right)^{3 / 2}},
$$

where we used $v \approx 1$. This coincides with (3) for a single proton when we take $\mathbf{R}_{a}=\mathbf{b}+(z-v t) \widehat{\mathbf{z}}$. Consider field strength (17) at the origin $z=0$. At times $t<b / \gamma$ the field is constant, while at $t \gg b / \gamma$ it decreases as $B_{\infty} \propto 1 / t^{3}$. At the time $t \approx b$ the ratio between these two is

$$
\frac{B_{0}}{B_{\infty}}=\frac{1}{\gamma^{3}} \ll 1
$$

which is a very small number $\left(\sim 10^{-6}\right.$ at RHIC).

In matter $\sigma>0$. Let me write the modified Bessel function appearing in (14) as follows:

$$
s K_{1}(s b)=\int_{0}^{\infty} \frac{J_{1}(x b) x^{2} d x}{x^{2}+s^{2}} .
$$

Substituting (19) into (14) and using (16), we have ( $v=1)$

$$
e \mathbf{B}=\frac{\alpha_{\mathrm{em}}}{\pi} \widehat{\mathbf{y}} \int_{0}^{\infty} d x \int_{-\infty}^{\infty} d \omega \frac{J_{1}(x b) x^{2}}{x^{2}-i \omega \sigma} e^{i \omega(z-t)} .
$$

Closing the contour in the lower half-plane of complex $\omega$ picks a pole at $\omega=-i x^{2} / \sigma$. We have

$$
\begin{aligned}
e \mathbf{B} & =\frac{2 \alpha_{\mathrm{em}}}{\sigma} \widehat{\mathbf{y}} \int_{0}^{\infty} d x x^{2} J_{1}(x b) e^{-\left(x^{2} / \sigma\right)(t-z)} \\
& =\widehat{\mathbf{y}} \frac{\alpha_{\mathrm{em}} b \sigma}{2(t-z)^{2}} e^{-b^{2} \sigma / 4(t-z)} .
\end{aligned}
$$


At $z=0$ this function vanishes at $t=0$ and $t \rightarrow \infty$ and has maximum at the time instant $t=b^{2} \sigma / 8$ which is much larger than $b / \gamma$. The value of the magnetic field at this time is

$$
e B_{\max }=\frac{32 e^{-2} \alpha_{\mathrm{em}}}{b^{3} \sigma}
$$

(Here $e$ is the base of natural logarithm.) This is smaller than the maximum field in vacuum

$$
\frac{B_{\max }}{B_{0}}=\frac{32 e^{-2}}{\sigma b \gamma}
$$

but is still a huge field. We compare the two solutions (17) and (21) in Figure 4. We see that in a conducting medium magnetic field stays for a long time.

One essential component is still missing in our arguments-time dependence of plasma properties due to its expansion. Let us now turn to this problem.

1.3.2. Magnetic Field in Expanding Medium. So far I treated quark-gluon plasma as a static medium. Expanding medium temperature and hence conductivity are functions of time. In Bjorken scenario [20], expansion is isentropic, that is, $n V=$ const, where $n$ is the particle number density and $V$ is plasma volume. Since $n \sim T^{3}$ and at early times expansion is onedimensional $V \sim t$ it follows that $T \propto t^{-1 / 3}$. (Eventually, we will consider the midrapidity region $z=0$, therefore distinction between the proper time and $t$ is not essential.) Equation (5) implies that $\sigma \sim t^{-1 / 3}$. I will parameterize conductivity as follows:

$$
\sigma(t)=\sigma_{0}\left(\frac{t_{0}}{t_{0}+t}\right)^{1 / 3}
$$

where I took $t_{0} \approx 0.5 \mathrm{fm}$ to be the initial time (or longitudinal size) of plasma evolution. Suppose that plasma lives for $10 \mathrm{fm} / \mathrm{c}$ and then undergoes phase transition to hadronic gas at $T_{c}$. Then employing (5) we estimate $\sigma_{0} \approx 16 \mathrm{MeV}$. Let me define another parameter that I will need in the forthcoming calculation:

$$
\beta=\frac{4 \sigma_{0}}{3 t_{0}} \approx 43 \frac{\mathrm{MeV}}{\mathrm{fm}} .
$$

Magnetic field in expanding medium is still governed by (8). As was explained in the preceding subsection, time evolution of magnetic field is quasi-static, which allows me to neglect the second time derivative. Let me introduce a new "time" variable $\rho$ as follows:

$$
\rho=\left(\frac{1+t}{t_{0}}\right)^{4 / 3}-1
$$

Field $\mathbf{B}(z, \mathbf{b}, \rho)$ satisfies equation

$$
\nabla^{2} \mathbf{B}=\beta \frac{\partial \mathbf{B}}{\partial \rho}-e v \nabla \times\{\widehat{\mathbf{z}} \delta[z-v t(\rho)] \delta(\mathbf{b})\},
$$

where

$$
t(\rho)=t_{0}\left[(\rho+1)^{3 / 4}-1\right]
$$

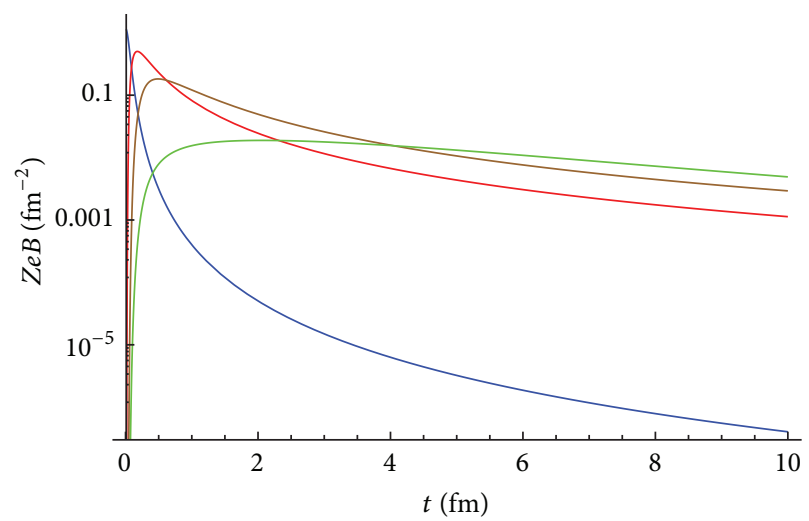

Figure 4: Relaxation of magnetic field at $z=0$ in vacuum (blue), in static conducting medium at $\sigma=5.8 \mathrm{MeV}$ (red) and at $\sigma=16 \mathrm{MeV}$ (brown), and in the expanding medium (green). Units of $B$ is $\mathrm{fm}^{-2} \approx$ $2 m_{\pi}^{2} \cdot b=7 \mathrm{fm}, Z=79$ (gold nucleus), $\gamma=100$ (RHIC).

Its solution can be written as

$$
\begin{aligned}
\mathbf{B}(z, \mathbf{b}, \rho)= & \int_{0}^{\rho} d \rho^{\prime} \int_{-\infty}^{\infty} d z \\
& \times \int d^{2} \mathbf{b}\left(\mathfrak{S}\left(z-z^{\prime}, \mathbf{b}-\mathbf{b}^{\prime}, \rho-\rho^{\prime}\right) e v\right. \\
& \times \nabla\{\widehat{\mathbf{z}} \delta[z-v t(\rho)] \delta(\mathbf{b})\}
\end{aligned}
$$

in terms of Green's function $\mathfrak{G}\left(z-z^{\prime}, \mathbf{b}-\mathbf{b}^{\prime}, \rho-\rho^{\prime}\right)$ satisfying

$$
\nabla^{2} \mathfrak{G}-\beta \frac{\partial \mathfrak{G}}{\partial \rho}=-\delta\left(z-z^{\prime}\right) \delta\left(\mathbf{b}-\mathbf{b}^{\prime}\right) \delta\left(\rho-\rho^{\prime}\right)
$$

To solve this equation we represent $\mathfrak{G}$ as three-dimensional Fourier integral with respect to the space coordinates and Laplace transform with respect to the "time" coordinate:

$$
\mathfrak{G}(z, \mathbf{b}, \rho)=\int \frac{d^{3} k}{(2 \pi)^{3}} e^{i\left(\mathbf{k}_{\perp} \cdot \mathbf{b}+k_{z} z\right)} \int_{C} \frac{d \lambda}{2 \pi i} e^{\lambda \rho} \frac{1}{k_{\perp}^{2}+k_{z}^{2}+\beta \lambda},
$$

with the contour $C$ running parallel to the imaginary axis to the right of all integrand singularities. Now I would like to write the expression in the curly brackets in (29) also as Fourier-Laplace expansion. To this end we calculate

$$
\begin{aligned}
f_{\mathbf{k}, \lambda}= & \int d^{2} \mathbf{b} \int_{-\infty}^{\infty} d z \\
& \times \int_{0}^{\infty} d \rho e^{-i\left(\mathbf{k}_{\perp} \cdot \mathbf{b}+k_{z} z\right)} e^{-\lambda \rho} \delta(z-v t(\rho)) \delta(\mathbf{b}) \\
= & \int_{0}^{\infty} d \rho e^{-i k_{z} v t_{0}\left[(\rho+1)^{3 / 4}-1\right]} e^{-\lambda \rho} .
\end{aligned}
$$


Therefore,

$$
\begin{aligned}
& e v \nabla \times\{\widehat{\mathbf{z}} \delta[z-v t(\rho)] \delta(\mathbf{b})\} \\
& =e v \int \frac{d^{3} k}{(2 \pi)^{3}} e^{i\left(\mathbf{k}_{\perp} \cdot \mathbf{b}+k_{z} z\right)} \\
& \quad \times \int_{\mathrm{C}} \frac{d \lambda}{2 \pi i} e^{\lambda \rho} i \mathbf{k}_{\perp} \times \widehat{\mathbf{z}} f_{\mathbf{k}, \lambda} .
\end{aligned}
$$

Substituting (31) and (34) into (29) we obtain upon integration over the volume and time as follows:

$$
\begin{aligned}
\mathbf{B}(z, \mathbf{b}, \rho)= & \int \frac{d^{3} k}{(2 \pi)^{3}} e^{i\left(\mathbf{k}_{\perp} \cdot \mathbf{b}+k_{z} z\right)} \\
& \times \int_{C} \frac{d \lambda}{2 \pi i} e^{\lambda \rho} \frac{e v i \mathbf{k}_{\perp} \times \widehat{\mathbf{z}}}{k_{\perp}^{2}+k_{z}^{2}+\beta \lambda} f_{\mathbf{k}, \lambda} \theta(\rho),
\end{aligned}
$$

where $\theta$ is the step function. Taking consequent integrals over $\lambda$ and $k_{z}$ gives

$$
\begin{aligned}
& \mathbf{B}(0, \mathbf{b}, \rho)=\frac{e v}{\beta} \int \frac{d^{2} k_{\perp}}{(2 \pi)^{2}} e^{i \mathbf{k}_{\perp} \cdot \mathbf{b}} \int_{-\infty}^{\infty} \frac{d k_{z}}{2 \pi} i \mathbf{k}_{\perp} \\
& \times \widehat{\mathbf{z}} \int_{0}^{\rho} d \rho^{\prime} e^{-i k_{z} v t_{0}\left[\left(\rho^{\prime}+1\right)^{3 / 4}-1\right]} \\
& \times e^{-\left(\left(k_{\perp}^{2}+k_{z}^{2}\right) / \beta\right)\left(\rho-\rho^{\prime}\right)} \\
&=\frac{e v}{\beta} \int \frac{d^{2} k_{\perp}}{(2 \pi)^{2}} e^{i \mathbf{k}_{\perp} \cdot \mathbf{b}} \frac{1}{2 \pi} i \mathbf{k}_{\perp} \\
& \times \widehat{\mathbf{z}} \int_{0}^{\rho} d \rho^{\prime} e^{-\left(k_{\perp}^{2} / \beta\right)\left(\rho-\rho^{\prime}\right)} \\
& \times \frac{\sqrt{\pi \beta}}{\sqrt{\rho-\rho^{\prime}}} e^{-v^{2} t_{0}^{2} \beta\left[\left(\rho^{\prime}+1\right)^{3 / 4}-1\right]^{2} / 4\left(\rho-\rho^{\prime}\right)} .
\end{aligned}
$$

Consider now $B_{y}$. Integrating over azimuthal angle $\phi$ and then over $k_{\perp}$ as in (13), (14) yields

$$
\begin{aligned}
& e B_{y}(0, \mathbf{b}, \rho) \\
& =\frac{\alpha_{\mathrm{em}} v b \beta^{3 / 2}}{2 \sqrt{\pi}} \int_{0}^{\rho} d \zeta \zeta^{-5 / 2} \\
& \quad \times e^{-\mathbf{b}^{2} \beta / 4 \zeta} e^{-v^{2} t_{0}^{2} \beta\left[(\rho-\zeta+1)^{3 / 4}-1\right]^{2} / 4 \zeta},
\end{aligned}
$$

where $\zeta=\rho-\rho^{\prime}$.

The results of a numerical calculation of (37) are shown in Figure 4. We see that expansion of plasma tends to increase the relaxation time, although this effect is rather modest. We conclude that due to finite electrical conductivity of QGP, magnetic field essentially freezes in the plasma for as long as plasma exists. Similar phenomenon, known as skin effect, exists in good conductors placed in time-varying magnetic field: conductors expel time dependent magnetic fields from conductor volume confining them into a thin layer of width $\delta \sim \omega^{-1 / 2}$ on the surface.
1.3.3. Diffusion of Magnetic Field in QGP. The dynamics of magnetic field relaxation in conducting plasma can be understood in a simple model [21]. Suppose at some initial time $t=0$ magnetic field $\mathbf{B}(0, \mathbf{r})$ permits the plasma. The problem is to find the time dependence of the field at $t>0$. In this model, the field sources turn off at $t=0$ and do not at all contribute to the field at $t>0$. Electromagnetic field is governed by the following equations:

$$
\begin{gathered}
\nabla \times \mathbf{B}=\mathbf{j}, \quad \mathbf{j}=\sigma \mathbf{E}, \\
\nabla \times \mathbf{E}=-\frac{\partial \mathbf{B}}{\partial t}, \quad \nabla \cdot \mathbf{B}=0,
\end{gathered}
$$

that lead to the diffusion equation for $\mathbf{B}$, after we neglect the second-time derivative as discussed previously as follows:

$$
\nabla^{2} \mathbf{B}=\sigma \frac{\partial \mathbf{B}}{\partial t} .
$$

For simplicity we treat electrical conductivity $\sigma$ as constant. Initial condition at $t=0$ reads

$$
\mathbf{B}(0, \mathbf{r})=\mathbf{B}_{0} e^{-\mathbf{b}^{2} / R^{2}},
$$

where the Gaussian profile is chosen for illustration purposes, and $R$ is the nuclear radius. Solution to the problem (39), (40) is

$$
\mathbf{B}(t, \mathbf{r})=\int d V^{\prime} \mathbf{B}\left(0, \mathbf{r}^{\prime}\right) \mathrm{G}\left(t, \mathbf{r}-\mathbf{r}^{\prime}\right),
$$

where Green's function is

$$
G(t, \mathbf{r})=\frac{1}{(4 \pi t / \sigma)^{3 / 2}} \exp \left[-\frac{\mathbf{r}^{2}}{4 t / \sigma}\right] .
$$

Integrating over the entire volume we derive

$$
\mathbf{B}(t, \mathbf{r})=\mathbf{B}_{0} \frac{R^{2}}{R^{2}+4 t / \sigma} \exp \left[-\frac{\mathbf{b}^{2}}{R^{2}+4 t / \sigma}\right] .
$$

It follows from (43) that as long as $t \ll t_{\text {relax }}$, where $t_{\text {relax }}$ is a characteristic time $t_{\text {relax }}=R^{2} \sigma / 4$ and magnetic field $\mathbf{B}$ is approximately time independent. This estimate is the same as the one we arrived at after (21).

In summary, magnetic field in quark-gluon plasma appears to be extremely strong and slowly varying function of time for most of the plasma lifetime. At RHIC it decreases from $e B \approx\left(2.5 m_{\pi}\right)^{2}$ right after the collision to $e B \approx\left(m_{\pi} / 4\right)^{2}$ at $t \approx 5 \mathrm{fm}$ see Figure 4 . This has a profound impact on all the processes occurring in QGP.

1.3.4. Schwinger Mechanism. Schwinger mechanism of pair production [3] is operative if electric field exceeds the critical value of $m^{2} / e$, where $m$ is mass of lightest electrically charged particle. Indeed, in order to excite a fermion out of the Dirac sea, electric force $e \mathbf{E}$ must do work along the path $d \boldsymbol{\ell}$ satisfying

$$
\int_{0}^{\ell} e \mathbf{E} \cdot d \boldsymbol{\ell}^{\prime}>2 m
$$


If $\mathbf{E}=$ const, then $E \gtrsim m / \ell e$. The maximal value of $\ell$ is the fermion Compton's wavelength $\ell \sim \lambda=1 / m$ implying that the minimum (or critical) value of electric field is

$$
E_{c}=\frac{m^{2}}{e}
$$

Notice that in stronger fields $\ell \sim m / e E<\lambda$. Figure 3 indicates that electron-positron pairs are certainly produced at RHIC. An important question then is the role of these pairs in the electromagnetic field relaxation in plasma. There are two associated effects: (i) before $e^{-} e^{+}$pairs thermalize, they contribute to the Foucault currents; (ii) after they thermalize, their density contributes to the polarization of plasma in electric field and hence to its conductivity.

Since space dimensions of QGP are much less than $\lambda_{e}=$ $380 \mathrm{fm}$, it may seem inevitable that space dependence of electric field (in addition to its time dependence) has a significant impact on the Schwinger process in heavy-ion collisions. However, this conclusion is premature. Indeed, suppose that electric field is a slow function of coordinates. Then $\mathbf{E}(\mathbf{r}) \approx \mathbf{E}(0)+\mathbf{r} \cdot \nabla \mathbf{E}(0)$. Work done by electric field is

$$
\int_{0}^{\ell} e \mathbf{E}(0) \cdot d \boldsymbol{\ell}^{\prime}+\int_{0}^{\ell}(\mathbf{r} \cdot \nabla) e \mathbf{E}(0) \cdot d \boldsymbol{\ell}^{\prime} \sim e E \ell+\frac{e \ell^{2} E(0)}{\lambda},
$$

where $\lambda$ is length scale describing space variation of electric field. In order that contribution of space variation to work be negligible, the second term in the r.h.s. of (46) must satisfy $e \ell^{2} E(0) / \lambda \ll m$. Employing the estimate $\ell \sim m / e E(0)$ that we obtained after (45) implies $m / e E(0) \lambda \ll 1$. Following [22] I define the inhomogeneity parameter

$$
\tilde{\gamma}=\frac{m}{\lambda e E}
$$

that describes the effect of spatial variation of electric field on the pair production rate. For electrons $m=0.5 \mathrm{MeV}$ in QGP $\lambda \sim 0.5 \mathrm{fm}$ at $e E \sim m_{\pi}^{2}$ we have $\tilde{\gamma} \sim 0.01$. Therefore, somewhat counter intuitively, electric field can be considered as spatially homogeneous. The same conclusion can be derived from results of [23]. Schwinger mechanism in spatially dependent electric fields was also discussed in [24, 25].

In view of smallness of $\widetilde{\gamma}$ one can employ the extensive literature on Schwinger effect in time-dependent spatiallyhomogeneous electric fields. As far as heavy-ion physics is concerned, the most comprehensive study has been done in $[6,26,27]$ who developed an approach to include the effect of backreaction. They argued that time evolution of electric field can be studied in adiabatic approximation and used the kinetic approach to study the time evolution. Their results are exhibited in Figure 5. Similar results were obtained in [28]. We observe that response time of the current density of Schwinger pairs $\sim 10^{4} \mathrm{fm} / \mathrm{c}$ is much larger than the plasma lifetime $\sim 10 \mathrm{fm} / \mathrm{c}$, and therefore no sizable electric current is generated.

In summary, strong electric field is generated in heavy-ion collisions in every event but averages to zero in a large event ensemble. This field exceeds the critical value for electrons and light $u, d$ quarks. However, during the plasma lifetime no significant current of Schwinger pairs is generated.

\section{Flow of Quark-Gluon Plasma in Strong Magnetic Field}

2.1. Azimuthal Asymmetry. Magnetic field is known to have a profound influence on kinetic properties of plasmas. Once the spherical symmetry is broken, distribution of particles in plasma is only axially symmetric with respect to the magnetic field direction. This symmetry, however, is not manifest in the plane span by magnetic field and the impact parameter vectors, namely, $x y$-plane in Figure 1. Charged particles moving along the magnetic field direction $y$ are not influenced by the magnetic Lorentz force, while those moving the $x z$-plane (i.e., the reaction plane) are affected the most. The result is azimuthally anisotropic flow of expanding plasma in the $x y$-plane even when initial plasma geometry is completely spherically symmetric. The effect of weak magnetic field on quark-gluon plasma flow was first considered in [29] who argued that magnetic field is able to enhance the azimuthal anisotropy of produced particles up to $30 \%$. This conclusion was reached by utilizing a solution of the magnetohydrodynamic equations in weak magnetic field.

A characteristic feature of the viscous pressure tensor in magnetic field is its azimuthal anisotropy. This anisotropy is the result of suppression of the momentum transfer in QGP in the direction perpendicular to the magnetic field. Its macroscopic manifestation is decrease of the viscous pressure tensor components in the plane perpendicular to the magnetic field, which coincides with the reaction plane in the heavy-ion phenomenology. Since Lorentz force vanishes in the direction parallel to the field, viscosity along that direction is not affected at all. In fact, the viscous pressure tensor component in the reaction plane is twice as small as the one in the field direction. As the result, transverse flow of QGP develops azimuthal anisotropy in presence of the magnetic field. Clearly, this anisotropy is completely different from the one generated by the anisotropic pressure gradients and exists even if the later is absent. In fact, because spherical symmetry in magnetic field is broken, viscous effects in plasma cannot be described by only two parameters: shear $\eta$ and bulk viscosity $\zeta$. Rather the viscous pressure tensor of magnetoactive plasma is characterized by seven viscosity coefficients, among which five are shear viscosities and two are bulk ones.

\subsection{Viscous Pressure in Strong Magnetic Field}

2.2.1. Viscosities from Kinetic Equation. Generally, calculation of the viscosities requires knowledge of the strong interaction dynamics of the QGP components. However, in strong magnetic field these interactions can be considered as a perturbation, and viscosities can be analytically calculated using the kinetic equation [30-33]. To apply this approach to QGP in strong magnetic field we start with kinetic equation for the distribution function $f$ of a quark flavor of charge $z e$ as follows:

$$
p^{\mu} \partial_{\mu} f=z e B^{\mu \nu} \frac{\partial f}{\partial u^{\mu}} u_{v}+\mathscr{C}[f, \ldots]
$$




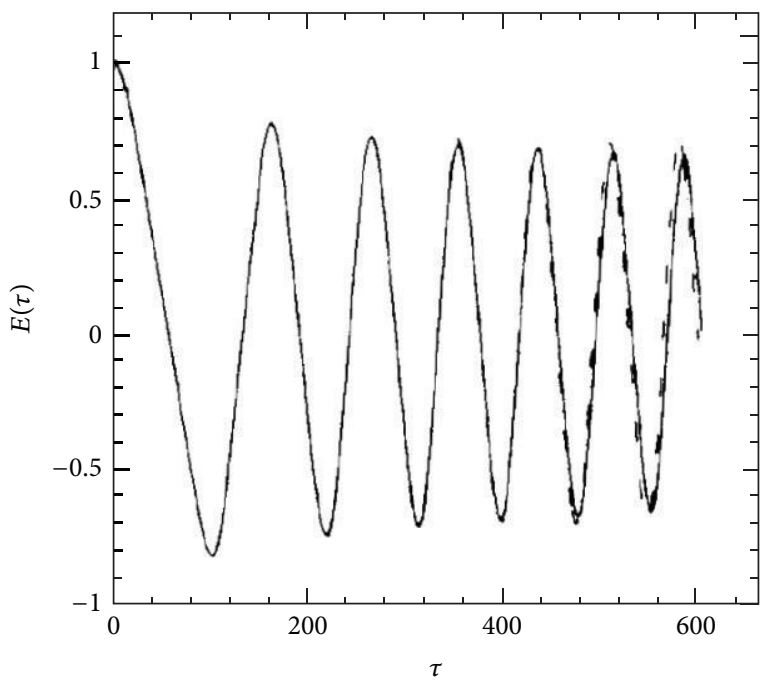

(a)

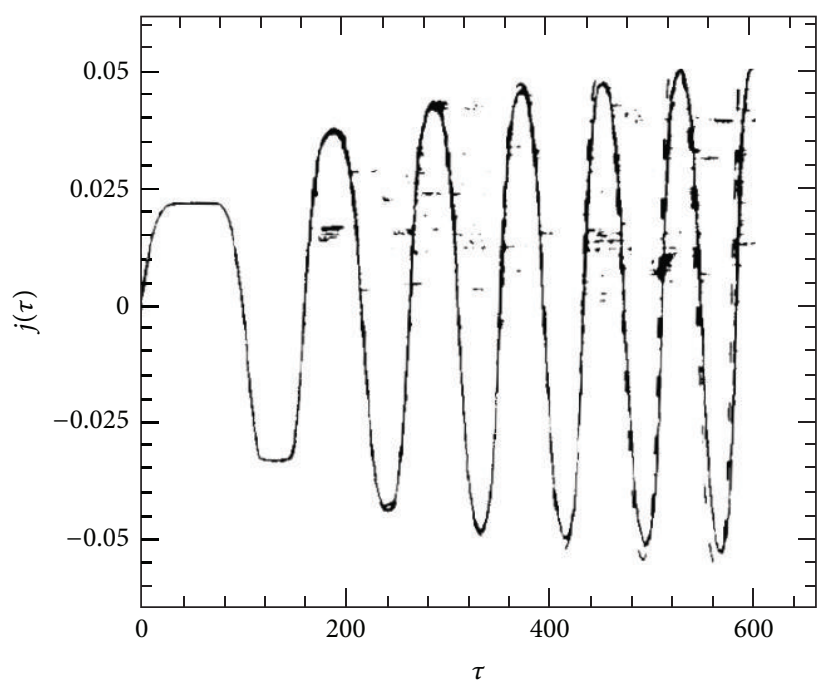

(b)

Figure 5: Time dependence of electric field due to the Schwinger mechanism back reaction and the corresponding electric current density of Schwinger pairs. Dimensionless time variable is defined as $\tau=t / \lambda$. For electrons $\lambda=380 \mathrm{fm}$. Plasma undergoes phase transition at about $\tau=1 / 38$. Adapted from [6].

where $\mathscr{C}$ is the collision integral and $B^{\mu \nu}$ is the electromagnetic tensor, which contains only magnetic field components in the laboratory frame. Ellipsis in the argument of $\mathscr{C}$ indicates the distribution functions of other quark flavors and gluons (I will omit them in the following). The equilibrium distribution reads

$$
f_{0}=\frac{\rho}{4 \pi m^{3} T K_{2}(\beta m)} e^{-\beta p \cdot U(x)},
$$

where $U(x)$ is the macroscopic velocity of the fluid, $p^{\mu}=$ $m u^{\mu}$ is particle momentum, $\beta=1 / T$, and $\rho$ is the mass density. Since $\left(\partial f_{0} / \partial u^{\mu}\right) \propto u_{\mu}$, the first term on the r.h.s. of (48) as well as the collision integral vanishes in equilibrium. Therefore, we can write the kinetic equation as an equation for $\delta f$ :

$$
p^{\mu} \partial_{\mu} f_{0}=z e B^{\mu \nu} \frac{\partial(\delta f)}{\partial u^{\mu}} u_{v}+\mathscr{C}[\delta f]
$$

where $\delta f$ is a deviation from equilibrium. Differentiating (49) we find

$$
\partial_{\mu} f_{0}=-f_{0} \frac{1}{T} p^{\lambda} \partial_{\mu} U_{\lambda}(x)
$$

Since $U^{\lambda}=\left(\gamma_{V}, \gamma_{V} \mathbf{V}\right)$ and $p^{\lambda}=(\varepsilon, \mathbf{p})=\left(\gamma_{v} m, \gamma_{v} m \mathbf{v}\right)$ it follows that

$$
p \cdot U=\frac{m}{\sqrt{1-v^{2}} \sqrt{1-V^{2}}}(1-\mathbf{v} \cdot \mathbf{V}) .
$$

Thus, in the comoving frame

$$
\left.\partial_{\mu} f_{0}\right|_{\mathbf{V}=0}=f_{0} \frac{1}{T} p_{\nu} \partial_{\mu} V^{\nu} .
$$

Substituting (53) in (50) yields

$$
-\frac{f_{0}}{T} p^{\mu} p^{\nu} V_{\mu \nu}=z e B^{\mu \nu} \frac{\partial(\delta f)}{\partial u^{\mu}} u_{\nu}+\mathscr{C}[\delta f],
$$

where I defined

$$
V_{\mu \nu}=\frac{1}{2}\left(\partial_{\mu} V_{\nu}+\partial_{\nu} V_{\mu}\right)
$$

and used $u^{\mu} u^{\nu} \partial_{\mu} V_{v}=u^{\mu} u^{\nu} V_{\mu v}$.

Since the time derivative of $f_{0}$ is irrelevant for the calculation of the viscosity I will drop it from the kinetic equation. All indices thus become the usual three-vector ones. To avoid confusion we will label them by the Greek letters from the beginning of the alphabet. Introducing $b_{\alpha \beta}=$ $B^{-1} \varepsilon_{\alpha \beta \gamma} B_{\gamma}$, we cast (54) in the form

$$
\frac{1}{T} p^{\alpha} u^{\beta} V_{\alpha \beta} f_{0}=-z e B b_{\alpha \beta} v^{\beta} \frac{\partial(\delta f)}{\partial v_{\alpha}} \frac{1}{\varepsilon}-\mathscr{C}[\delta f] .
$$

The viscous pressure generated by a deviation from equilibrium is given by the tensor

$$
-\Pi_{\alpha \beta}=\int p_{\alpha} p_{\beta} \delta f \frac{d^{3} p}{\varepsilon} .
$$

Effectively it can be parameterized in terms of the viscosity coefficients as follows (we neglect the bulk viscosities):

$$
\Pi_{\alpha \beta}=\sum_{n=0}^{4} \eta_{n} V_{\alpha \beta}^{(n)},
$$


where the linearly independent tensors $V_{\alpha \beta}^{(n)}$ are given by

$$
\begin{gathered}
V_{\alpha \beta}^{(0)}=\left(3 b_{\alpha} b_{\beta}-\delta_{\alpha \beta}\right)\left(b_{\gamma} b_{\delta} V_{\gamma \delta}-\frac{1}{3} \nabla \cdot \mathbf{v}\right) \\
V_{\alpha \beta}^{(1)}=2 V_{\alpha \beta}+\delta_{\alpha \beta} V_{\gamma \delta} b_{\gamma} b_{\delta}-2 V_{\alpha \gamma} b_{\gamma} b_{\beta}-2 V_{\beta \gamma} b_{\gamma} b_{\alpha} \\
+\left(b_{\alpha} b_{\beta}-\delta_{\alpha \beta}\right) \nabla \cdot \mathbf{V}+b_{\alpha} b_{\beta} V_{\gamma \delta} b_{\gamma} b_{\delta} \\
V_{\alpha \beta}^{(2)}=2\left(V_{\alpha \gamma} b_{\beta \gamma}+V_{\beta \gamma} b_{\alpha \gamma}-V_{\gamma \delta} b_{\alpha \gamma} b_{\beta} b_{\delta}\right) \\
V_{\alpha \beta}^{(3)}=V_{\alpha \gamma} b_{\beta \gamma}+V_{\beta \gamma} b_{\alpha \gamma}-V_{\gamma \delta} b_{\alpha \delta} b_{\alpha \gamma} b_{\beta} b_{\delta}-V_{\gamma \delta} b_{\beta \gamma} b_{\alpha} b_{\delta} \\
V_{\alpha \beta}^{(4)}=2\left(V_{\gamma \delta} b_{\alpha \delta} b_{\alpha \gamma} b_{\beta} b_{\delta}+V_{\gamma \delta} b_{\beta \gamma} b_{\alpha} b_{\delta}\right)
\end{gathered}
$$

Here $b_{\alpha}$ is a unit vector in the direction of magnetic field. For the calculation of the shear viscosities $\eta_{n}, n=0, \ldots, 4$ we can set $\nabla \cdot \mathbf{V}=0$ and $V_{\alpha \beta} b_{\alpha} b_{\beta}=0$.

Let us expand $\delta f$ to the second order in velocities in terms of the tensors $V_{\alpha \beta}^{(n)}$ as follows:

$$
\delta f=\sum_{n=0}^{4} g_{n} V_{\alpha \beta}^{(n)} v^{\alpha} v^{\beta} .
$$

Then, substituting (60) into (58) and requiring consistency of (57) and (58) yield

$$
\eta_{n}=-\frac{2}{15} \int \varepsilon v^{4} g_{n} d^{3} p
$$

This gives the viscosities in the magnetic field in terms of the deviation of the distribution function from equilibrium. Transition to the nonrelativistic limit in (61) is achieved by the replacement $\varepsilon \rightarrow m[30]$.

2.2.2. Collisionless Plasma. In strong magnetic field we can determine $g_{n}$ by the method of consecutive approximations. Writing $\delta f=\delta f^{(1)}+\delta f^{(2)}$ and substituting into (56), we find

$$
\frac{1}{T} p^{\alpha} \nu^{\beta} V_{\alpha \beta} f_{0}=-z e B b_{\alpha \beta} \nu^{\beta} \frac{\partial\left(\delta f^{(1)}+\delta f^{(2)}\right)}{\partial v_{\alpha}} \frac{1}{\varepsilon}+\mathscr{C}\left[\delta f^{(1)}\right] .
$$

Here I assumed that the deviation from equilibrium due to the strong magnetic field is much larger than due to the particle collisions. The explicit form of $\mathscr{C}$ is determined by the strong interaction dynamicsbut drops off the equation in the leading oder. The first correction to the equilibrium distribution obeys the equation

$$
\frac{1}{T} p_{\alpha} v_{\beta} V_{\alpha \beta} f_{0}=-z e B b_{\alpha \beta} v_{\beta} \frac{\partial \delta f^{(1)}}{\partial v_{\alpha}} \frac{1}{\varepsilon} .
$$

Using (60), we get

$$
b_{\alpha \beta} v_{\beta} \frac{\partial \delta f^{(1)}}{\partial v_{\alpha}}=2 b_{\alpha \beta} v_{\beta} \sum_{n=0}^{4} g_{n} V_{\alpha \gamma}^{(n)} v_{\gamma} .
$$

Substituting (64) into (63) and using (59a), (59b), (59c), (59d), and (59e) yield

$$
\begin{aligned}
\frac{\varepsilon}{T z e B} p_{\alpha} v_{\beta} V_{\alpha \beta} f_{0}=- & 2 b_{\beta \gamma} v_{\alpha} v_{\nu} \\
\times[ & g_{1}\left(2 V_{\alpha \beta}-2 V_{\beta \gamma} b_{\gamma} b_{\alpha}\right)+2 g_{2} V_{\beta \gamma} b_{\gamma} b_{\alpha} \\
& +g_{3}\left(V_{\alpha \gamma} b_{\beta \gamma}+V_{\beta \gamma} b_{\alpha \gamma}-V_{\gamma \delta} b_{\alpha} b_{\delta}\right) \\
& \left.+2 g_{4} V_{\gamma \delta} b_{\beta \gamma} b_{\alpha} b_{\delta}\right],
\end{aligned}
$$

where I used the following identities $b_{\alpha \beta} b_{\alpha}=b_{\alpha \beta} b_{\beta}=$ $b_{\alpha \beta} v_{\alpha} v_{\beta}=0$. Clearly, (65) is satisfied only if $g_{1}=g_{2}=0$. Concerning the other two coefficients, we use the identities

$$
\begin{gathered}
b_{\alpha \beta} b_{\beta \gamma}=b_{\gamma} b_{\alpha}-\delta_{\alpha \gamma} b^{2} \\
\varepsilon_{\alpha \beta \gamma} \varepsilon_{\delta \epsilon \zeta}=\delta_{\alpha \delta}\left(\delta_{\beta \epsilon} \delta_{\gamma \zeta}-\delta_{\beta \zeta} \delta_{\gamma \epsilon}\right)-\delta_{\alpha \epsilon}\left(\delta_{\beta \delta} \delta_{\gamma \zeta}-\delta_{\beta \zeta} \delta_{\gamma \delta}\right) \\
+\delta_{\alpha \zeta}\left(\delta_{\beta \delta} \delta_{\gamma \epsilon}-\delta_{\beta \zeta} \delta_{\gamma \delta}\right)
\end{gathered}
$$

that we substitute into (65) to derive

$$
\begin{aligned}
-\frac{\varepsilon}{2 T z e B} p^{\alpha} v^{\beta} V_{\alpha \beta} f_{0}= & g_{3}\left[2 V_{\alpha \beta} b_{\alpha} b_{\beta}-4 V_{\alpha \beta} v_{\alpha} b_{\beta}(\mathbf{b} \cdot \mathbf{v})\right] \\
& +2 g_{4} V_{\alpha \beta} v_{\alpha} b_{\beta}(\mathbf{b} \cdot \mathbf{v}) .
\end{aligned}
$$

Since $p_{\alpha}=\varepsilon v_{\alpha}$, we obtain

$$
g_{3}=\frac{g_{4}}{2}=-\frac{\varepsilon^{2} f_{0}}{4 T z e B} .
$$

Using (49), (68) in (61) in the comoving frame (of course $\eta_{n} \mathrm{~s}$ do not depend on the frame choice) and integrating using 3.547 .9 of [34], we derive [35]

$$
\eta_{3}=\frac{K_{3}(\beta m)}{K_{2}(\beta m)} \frac{\rho T}{2 z e B} .
$$

The nonrelativistic limit corresponds to $m \gg T$ in which case we get

$$
\eta_{3}^{\mathrm{NR}}=\frac{\rho T}{2 z e B} .
$$

In the opposite ultrarelativistic case $m \ll T$ (hightemperature plasma)

$$
\eta_{3}^{\mathrm{UR}}=\frac{2 n T^{2}}{z e B},
$$

where $n=\rho / m$ is the number density.

2.2.3. Contribution of Collisions. In the relaxation-time approximation we can write the collision integral as

$$
\mathscr{C}[\delta f]=-\nu \delta f
$$


where $v$ is an effective collision rate. Strong field limit means that

$$
\omega_{B} \gg \nu,
$$

where $\omega_{B}=z e B / \varepsilon$ is the synchrotron frequency. Whether $v$ itself is function of the field depends on the relation between the Larmor radius $r_{B}=v_{T} / \omega_{B}$, where $v_{T}$ is the particle velocity in the plane orthogonal to $\mathbf{B}$ and the Debye radius $r_{D}$. If

$$
r_{B} \gg r_{D}
$$

then the effect of the field on the collision rate $v$ can be neglected [30]. Assuming that (74) is satisfied, the collision rate reads

$$
v=n v \sigma_{t}
$$

where $\sigma_{t}$ is the transport cross-section, which is a function of the saturation momentum $Q_{s}[36,37]$. We estimate $\sigma_{t} \sim$ $\alpha_{s}^{2} / Q_{s}^{2}$, with $Q_{s} \sim 1 \mathrm{GeV}$ and $n=P / T$ with pressure $\alpha_{s}^{2} P \sim 1 \mathrm{GeV} / \mathrm{fm}^{3}$; we get $v \sim 40 \mathrm{MeV}$. Inequality (73) is well satisfied since $e B \simeq m_{\pi}^{2}[5,11]$, and $m$ is in the range between the current and the constituent quark masses. On the other hand, applicability of the condition (74) is marginal and is very sensitive to the interaction details. In this section we assume that (74) holds in order to obtain the analytic solution. Additionally, the general condition for the applicability of the hydrodynamic approach $\ell=1 / \nu \ll L$, where $\ell$ is the mean free path and $L$ is the plasma size is assumed to hold. Altogether we have $r_{D} \ll r_{B} \ll \ell \ll L$.

Equation for the second correction to the equilibrium distribution $\delta f^{(2)}$ follows from (62) after substitution (72)

$$
\frac{z e B}{\varepsilon} b_{\alpha \beta} v_{\beta} \frac{\partial \delta f^{(2)}}{\partial v_{\alpha}}=-v \delta f^{(1)} \text {. }
$$

Now, plugging

$$
\begin{aligned}
& \delta f^{(1)}=\left[g_{3} V_{\alpha \beta}^{(3)}+g_{4} V_{\alpha \beta}^{(4)}\right] v_{\alpha} v_{\beta}, \\
& \delta f^{(2)}=\left[g_{1} V_{\alpha \beta}^{(1)}+g_{2} V_{\alpha \beta}^{(2)}\right] v_{\alpha} v_{\beta},
\end{aligned}
$$

into (76) yields

$$
\begin{aligned}
\frac{2 z e B}{\varepsilon}\left\{g_{1}\left[2 V_{\beta \alpha} b_{\alpha \gamma} v_{\beta} v_{\gamma}-2 V_{\beta \alpha} b_{\alpha \gamma} v_{\beta} v_{\gamma}(\mathbf{v} \cdot \mathbf{b})\right]\right. \\
\left.+2 g_{2} V_{\beta \alpha} b_{\alpha \gamma} v_{\beta} v_{\gamma}(\mathbf{v} \cdot \mathbf{b})\right\} \\
=-v g_{3}\left\{-2 V_{\beta \alpha} b_{\alpha \gamma} v_{\beta} v_{\gamma}-6 V_{\beta \alpha} b_{\alpha \gamma} v_{\beta} v_{\gamma}(\mathbf{v} \cdot \mathbf{b})\right\},
\end{aligned}
$$

where I used $g_{4}=2 g_{3}$. It follows that

$$
g_{1}=\frac{g_{2}}{4}=\frac{\nu \gamma_{\nu} g_{3}}{2 \omega_{B}} .
$$

With the help of (80), (49), and (65) we obtain [35]

$$
\eta_{1}=\frac{\eta_{2}}{4}=\frac{8}{5 \sqrt{2 \pi}} \frac{\rho^{2} \sigma_{t} T^{3 / 2}}{(z e B)^{2} m^{1 / 2}} \frac{K_{7 / 2}(\beta m)}{K_{2}(\beta m)} .
$$

2.3. Azimuthal Asymmetry of Transverse Flow: A Simple Model. To illustrate the effect of the magnetic field on the viscous flow of the electrically charged component of the quark-gluon plasma I will assume that the flow is nonrelativistic and use the Navier-Stokes equations that read

$$
\rho\left(\frac{\partial V_{\alpha}}{\partial t}+V_{\beta} \frac{\partial V_{\alpha}}{\partial x_{\beta}}\right)=-\frac{\partial P}{\partial x_{\alpha}}+\frac{\partial \Pi_{\alpha \beta}}{\partial x_{\beta}},
$$

where $\Pi_{\alpha \beta}$ is the viscous pressure tensor, $\rho=m n$ is mass density, and $P$ is pressure. I will additionally assume that the flow is nonturbulent and that the plasma is non-compressible. The former assumption amounts to dropping the terms nonlinear in velocity, while the later implies vanishing divergence of velocity

$$
\nabla \cdot \mathbf{V}=0
$$

Because of the approximate boost invariance of the heavyion collisions, we can restrict our attention to the two dimensional flow in the $x y$-plane corresponding to the central rapidity region.

The viscous pressure tensor in vanishing magnetic field is isotropic in the $x y$-plane and is given by

$$
\Pi_{\alpha \beta}^{0}=\eta\left(\frac{\partial V_{\alpha}}{\partial x_{\beta}}+\frac{\partial V_{\beta}}{\partial x_{\alpha}}\right)=2 \eta\left(\begin{array}{cc}
V_{x x} & V_{x y} \\
V_{y x} & V_{y y}
\end{array}\right)
$$

where the superscript 0 indicates absence of the magnetic field. In the opposite case of very strong magnetic field the viscous pressure tensor has a different form (58). Neglecting all $\eta_{n}$ with $n \geq 1$, we can write

$$
\Pi_{\alpha \beta}^{\infty}=\eta_{0}\left(\begin{array}{cc}
-V_{y y} & 0 \\
0 & 2 V_{y y}
\end{array}\right)=2 \eta_{0}\left(\begin{array}{cc}
\frac{1}{2} V_{x x} & 0 \\
0 & V_{y y}
\end{array}\right),
$$

where we also used (82). Notice that $\Pi_{x x}^{\infty}=(1 / 2) \Pi_{y y}^{\infty}=$ $(1 / 2) \Pi_{x x}^{0}$ indicating that the plasma flows in the direction perpendicular to the magnetic field with twice as small viscosity as in the direction of the field. The later is not affected by the field at all, because the Lorentz force vanishes in the field direction. Substituting (84) into (81) we derive the following two equations characterizing the plasma velocity in the strong magnetic field [35]:

$$
\rho \frac{\partial V_{x}}{\partial t}=-\frac{\partial P}{\partial x}+\eta_{0} \frac{\partial^{2} V_{x}}{\partial x^{2}}, \quad \rho \frac{\partial V_{y}}{\partial t}=-\frac{\partial P}{\partial y}+2 \eta_{0} \frac{\partial^{2} V_{y}}{\partial y^{2}}
$$

Additionally, we need to set the initial conditions

$$
\left.V_{x}\right|_{t=0}=\varphi_{1}(x, y),\left.\quad V_{y}\right|_{t=0}=\varphi_{2}(x, y) .
$$


The solution to the the problem (85), (86) is

$$
\begin{aligned}
V_{x}(x, y, t) \\
=\int_{-\infty}^{\infty} d x^{\prime} \varphi_{1}\left(x^{\prime}, y\right) G_{1 / 2}\left(x-x^{\prime}, t\right) \\
\quad-\frac{1}{\rho} \int_{0}^{t} d t^{\prime} \int_{-\infty}^{\infty} d x^{\prime} G_{1 / 2}\left(x-x^{\prime}, t-t^{\prime}\right) \frac{\partial P\left(x^{\prime}, y, t^{\prime}\right)}{\partial x^{\prime}},
\end{aligned}
$$

$$
\begin{aligned}
V_{y}(x, y, t) \\
=\int_{-\infty}^{\infty} d y^{\prime} \varphi_{2}\left(\mathrm{x}, y^{\prime}\right) G_{1}\left(y-y^{\prime}, t\right) \\
\quad-\frac{1}{\rho} \int_{0}^{t} d t^{\prime} \int_{-\infty}^{\infty} d y^{\prime} G_{1}\left(y-y^{\prime}, t-t^{\prime}\right) \frac{\partial P\left(x, y^{\prime}, t^{\prime}\right)}{\partial y^{\prime}} .
\end{aligned}
$$

Here Green's function is given by

$$
G_{k}(y, t)=\frac{1}{\sqrt{4 \pi a^{2} k t}} e^{-y^{2} / 4 a^{2} k t}
$$

and the diffusion coefficient by

$$
a^{2}=\frac{2 \eta_{0}}{\rho}
$$

Suppose that the pressure is isotropic; that is, it depends on the coordinates $x, y$ only via the radial coordinate $r=$ $\sqrt{x^{2}+y^{2}}$; accordingly we pass from the integration variables $x^{\prime}$ and $y^{\prime}$ to $r^{\prime}$ in (87a) and (87b) correspondingly. At later times we can expand Green's function (88) in inverse powers of $t$. The first terms in the r.h.s. of (87a) and (87b) are subleading, and we obtain

$$
\begin{aligned}
& V_{x}(x, y, t) \\
& \quad \approx-\frac{1}{\rho} \int_{0}^{t} d s \int_{-\infty}^{\infty} d r \frac{1}{\sqrt{2 \pi a^{2} s}} \frac{\partial P(r, t-s)}{\partial r} \\
& \quad=-\frac{1}{\rho} \int_{0}^{t} d s \frac{1}{\sqrt{2 \pi a^{2} s}}[P(R(s), t-s)-P(0, t-s)]
\end{aligned}
$$

and by the same token

$$
\begin{aligned}
& V_{y}(x, y, t) \\
& \quad \approx-\frac{1}{\rho} \int_{0}^{t} d s \frac{1}{\sqrt{4 \pi a^{2} s}}[P(R(s), t-s)-P(0, t-s)],
\end{aligned}
$$

where $R(t)$ denotes the boundary beyond which the density of the plasma is below the critical value. We observe that $V_{x} / V_{y}=\sqrt{2}$. Consequently, the azimuthal anisotropy of the hydrodynamic flow is [35]

$$
\frac{V_{x}^{2}-V_{y}^{2}}{V_{x}^{2}+V_{y}^{2}}=\frac{1-(1 / 2)}{1+(1 / 2)}=\frac{1}{3} .
$$

Since I assumed that the initial conditions and the pressure are isotropic, the azimuthal asymmetry (91) is generated exclusively by the magnetic field.

We see that at later times after the heavy-ion collision, flow velocity is proportional to $\eta_{0}^{-1 / 2}$, where $\eta_{0}$ is the finite shear viscosity coefficient; see (87a) and (87b). If the system is such that in absence of the magnetic field it were azimuthally symmetric, then the magnetic field induces azimuthal asymmetry of $1 / 3$; see (91). The effect of the magnetic field on flow is strong and must be taken into account in phenomenological applications. Neglect of the contribution by the magnetic field leads to underestimation of the phenomenological value of viscosity extracted from the data [38-40]. In other words, the more viscous QGP in magnetic field produces the same azimuthal anisotropy as a less viscous QGP in vacuum.

A model that I considered in this section to illustrate the effect of the magnetic field on the azimuthal anisotropy of a viscous fluid flow does not take into account many important features of a realistic heavy-ion collision. To be sure, a comprehensive approach must involve numerical solution of the relativistic magnetohydrodynamic equations with a realistic geometry. A potentially important effect that I have not considered here is plasma instabilities [41, 42], which warrant further investigation.

The structure of the viscous stress tensor in very strong magnetic field (84) is general, model independent. However, as explained, the precise amount of the azimuthal anisotropy that it generates cannot be determined without taking into account many important effects. Even so, I draw the reader's attention to the fact that analysis of [29] using quite different arguments arrived at similar conclusion. Although a more quantitive numerical calculation is certainly required before a final conclusion can be made, it looks very plausible that the QGP viscosity is significantly higher than the presently accepted value extracted without taking into account the magnetic field effect [38-40] and is perhaps closer to the value calculated using the perturbative theory $[43,44]$.

\section{Energy Loss and Polarization due to Synchrotron Radiation}

3.1. Radiation of Fast Quark in Magnetic Field. General problem of charged fermion radiation in external magnetic field was solved in [45-47]. It has important applications in collider physics; see, for example, $[48,49]$. In heavy-ion phenomenology, synchrotron radiation provides one of the mechanisms of energy loss in quark-gluon plasma, which is an important probe of QGP $[50,51]$ (synchrotron radiation in chromo-magnetic fields was discussed in [52-54]).

A typical diagram contributing to the synchrotron radiation, that is, radiation in external magnetic field, by a quark is shown in Figure 6 [21]. This diagram is proportional to $(e B)^{n}$, where $n$ is the number of external field lines. In strong field, powers of $e B$ must be summed up, which may be accomplished by exactly solving the Dirac equation for the relativistic fermion and then calculating the matrix element for the transition $q \rightarrow q+g$. Such calculation has been done in QED for some special cases including the homogeneous 


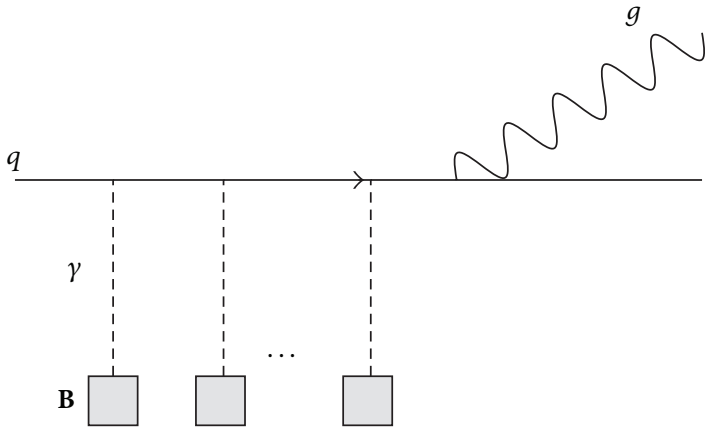

FIGURE 6: A typical diagram contributing to the synchrotron radiation by a quark.

constant field and can be readily generalized for gluon radiation. Intensity of the radiation can be expressed via the invariant parameter $\chi$ defined as

$$
\chi^{2}=-\frac{\alpha_{\mathrm{em}} Z_{q}^{2} \hbar^{3}}{m^{6}}\left(F_{\mu \nu} p^{\nu}\right)^{2}=\frac{\alpha_{\mathrm{em}} Z_{q}^{2} \hbar^{3}}{m^{6}}(\mathbf{p} \times \mathbf{B})^{2},
$$

where the initial quark 4-momentum is $p^{\mu}=(\varepsilon, \mathbf{p})$ and $Z_{q}$ is the quark charge in units of the absolute value of the electron charge $e$. At high energies,

$$
\chi \approx \frac{Z_{q} B \varepsilon}{B_{c} m} .
$$

The regime of weak fields corresponds to $\chi \ll 1$, while in strong fields $\chi \gg 1$. In our case, $e B / e B_{c} \approx\left(m_{\pi} / m_{u}\right)^{2} \gg 1$ (at RHIC), and therefore $\chi \gg 1$. In terms of $\chi$, spectrum of radiated gluons of frequency $\omega$ can be written as [45]

$$
\begin{aligned}
\frac{d I}{d \omega}= & -\alpha_{s} C_{F} \frac{m^{2} \omega}{\varepsilon^{2}} \\
& \times\left\{\int_{x}^{\infty} \operatorname{Ai}(\xi) d \xi+\left(\frac{2}{x}+\frac{\omega}{\varepsilon} \chi x^{1 / 2}\right) \mathrm{Ai}^{\prime}(x)\right\},
\end{aligned}
$$

where $I$ is the intensity

$$
x=\left(\frac{\hbar \omega}{\varepsilon^{\prime} \chi}\right)^{2 / 3},
$$

and $\varepsilon^{\prime}$ is quark's energy in the final state. Ai is the Ayri function. Equation (94) is valid under the assumption that the initial quark remains ultrarelativistic, which implies that the energy loss due to the synchrotron radiation $\Delta \varepsilon$ should be small compared to the quark energy itself $\Delta \varepsilon \ll \varepsilon$.

Energy loss by a relativistic quark per unit length is given by $[48]$

$$
\begin{aligned}
\frac{d \varepsilon}{d l} & =-\int_{0}^{\infty} d \omega \frac{d I}{d \omega} \\
& =\alpha_{s} C_{F} \frac{m^{2} \chi^{2}}{2} \int_{0}^{\infty} \frac{4+5 \chi x^{3 / 2}+4 \chi^{2} x^{3}}{\left(1+\chi x^{3 / 2}\right)^{4}} \mathrm{Ai}^{\prime}(x) x d x .
\end{aligned}
$$

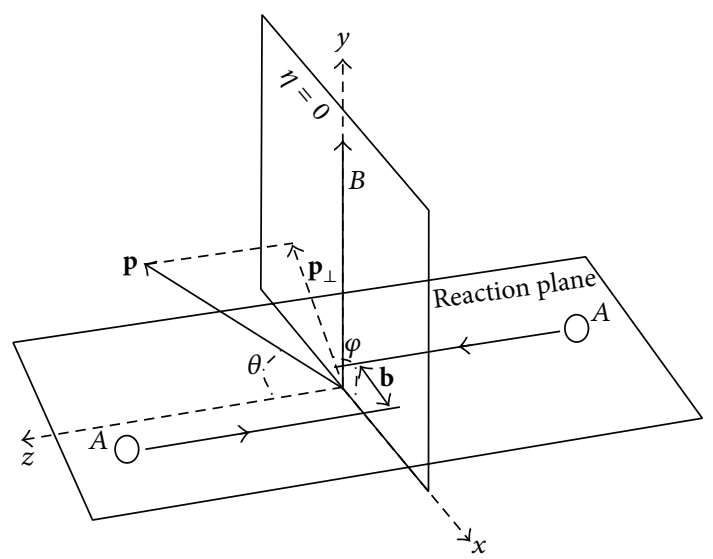

FIgURE 7: Geometry of a heavy-ion collision. p denotes momentum of a fast quark. Two orthogonal planes are the reaction plane span by the initial heavy-ion momenta and the mid rapidity plane $\theta=\pi / 2$, which is labeled as $\eta=0$.

In two interesting limits, energy loss behaves quite differently. At $\eta=\varphi=0$ we have [48]

$$
\begin{gathered}
\frac{d \varepsilon}{d l}=-\frac{2 \alpha_{s} \hbar C_{F}\left(Z_{q} e B\right)^{2} \varepsilon^{2}}{3 m^{4}}, \quad \chi \ll 1, \\
\frac{d \varepsilon}{d l}=-0.37 \alpha_{s} \hbar^{-1 / 3} C_{F}\left(Z_{q} e B \varepsilon\right)^{2 / 3}, \quad x \gg 1 .
\end{gathered}
$$

In the strong field limit energy loss is independent of the quark mass, whereas in the weak field case it decreases as $m^{-4}$. Since $\chi \propto \hbar$, limit of $\chi \ll 1$ corresponds to the classical energy loss.

To apply this result to heavy-ion collisions we need to write down the invariant $\chi$ in a suitable kinematic variables. The geometry of a heavy-ion collision is depicted in Figure 7. Magnetic field $\mathbf{B}$ is orthogonal to the reaction plane span by the impact parameter vector $\mathbf{b}$ and the collision axis $(z$-axis). For a quark of momentum $\mathbf{p}$ we define the polar angle $\theta$ with respect to the $z$-axis and azimuthal angle $\varphi$ with respect to the reaction plane. In this notation, $\mathbf{B}=B \widehat{\mathbf{y}}$ and $\mathbf{p}=p_{z} \widehat{\mathbf{z}}+$ $p_{\perp}(\widehat{\mathbf{x}} \cos \varphi+\widehat{\mathbf{y}} \sin \varphi)$, where $p_{\perp}=|\mathbf{p}| \sin \theta \approx \varepsilon \sin \theta$. Thus, $(\mathbf{B} \times \mathbf{p})^{2}=B^{2}\left(p_{z}^{2}+p_{\perp}^{2} \cos ^{2} \varphi\right)$. Conventionally, one expresses the longitudinal momentum and energy using the rapidity $\eta$ as $\varepsilon=m_{\perp} \cosh \eta$ and $p_{z}=m_{\perp} \sinh \eta$, where $m_{\perp}^{2}=m^{2}+p_{\perp}^{2}$. We have

$$
\chi^{2}=\frac{\hbar^{2}(e B)^{2}}{m^{6}} p_{\perp}^{2}\left(\sinh ^{2} \eta+\cos ^{2} \varphi\right)
$$

In Figure 8 a numerical calculation of the energy loss per unit length in a constant magnetic field using (96) and (98) is shown [21]. We see that energy loss of a $u$ quark with $p_{\perp}=10 \mathrm{GeV}$ is about $0.2 \mathrm{GeV} / \mathrm{fm}$ at RHIC and $1.3 \mathrm{GeV} / \mathrm{fm}$ at LHC. This corresponds to the loss of $10 \%$ and $65 \%$ of its initial energy after traveling $5 \mathrm{fm}$ at RHIC and LHC, respectively. Therefore, energy loss due to the synchrotron radiation at LHC gives a phenomenologically important contribution to the total energy loss. 


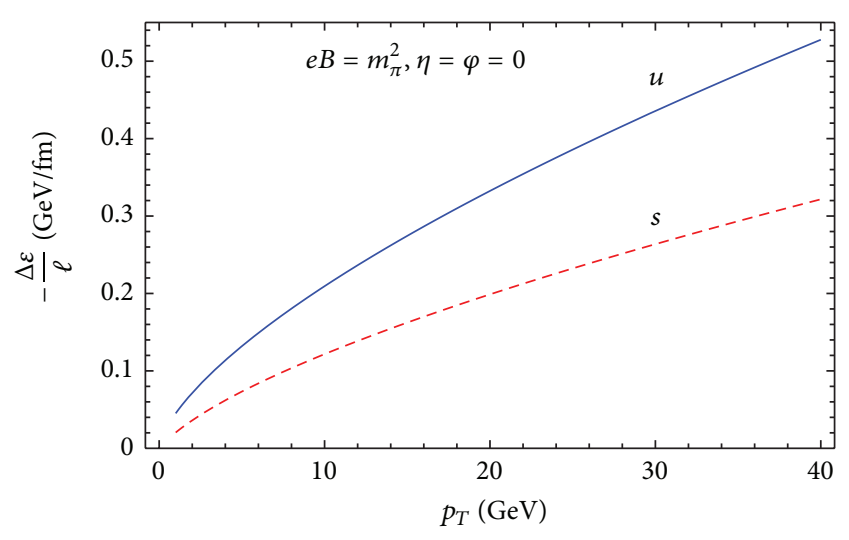

(a)

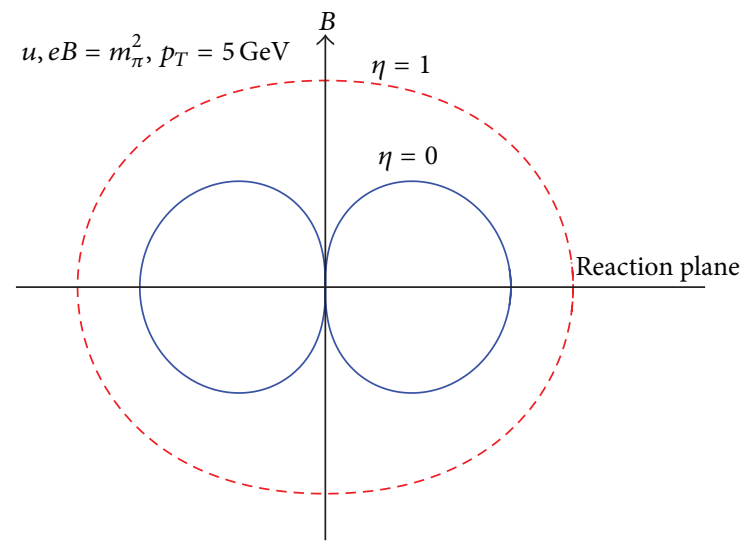

(c)

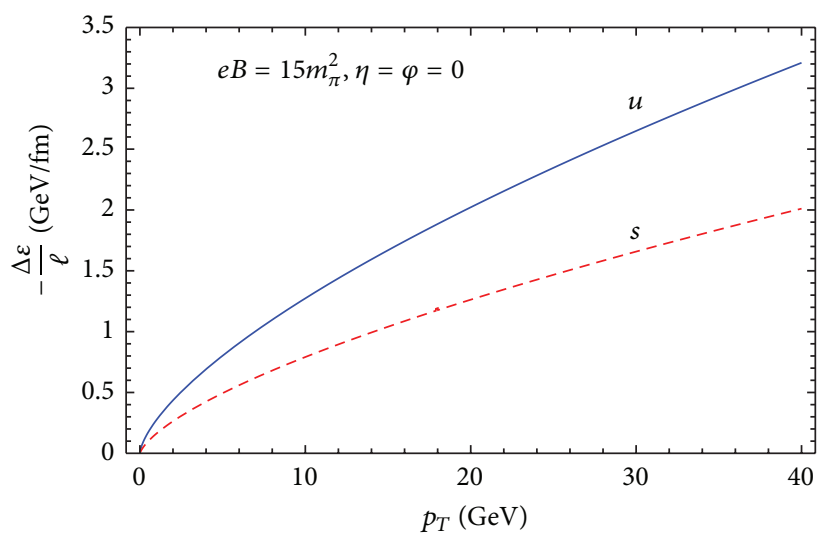

(b)

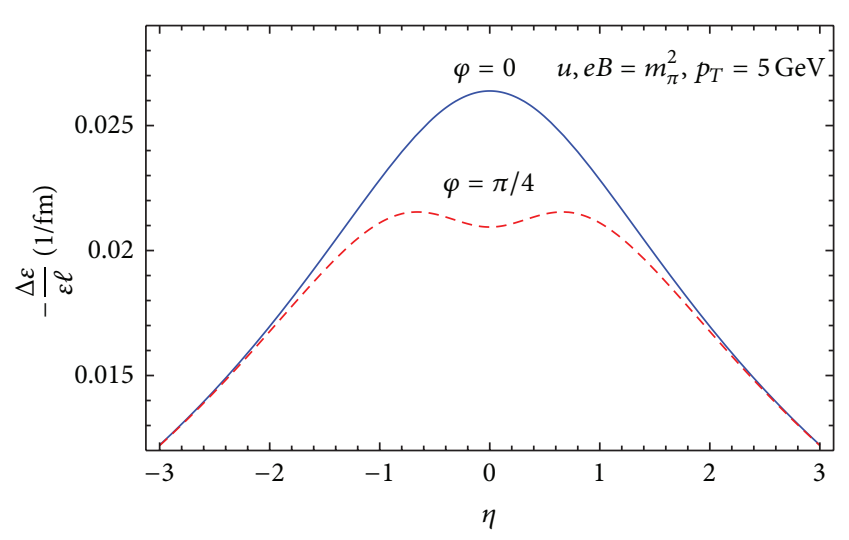

(d)

FIGURE 8: Energy loss per unit length by quarks moving in constant external magnetic field as a function of (a) $p_{T}$ at RHIC at $\eta=\varphi=0$, (b) $p_{T}$ at LHC at $\eta=\varphi=0$, (c) azimuthal angle $\varphi$ with respect to the reaction plane for $p_{T}=5 \mathrm{GeV}$ and $\eta=0$, 1 , and (d) fractional energy loss versus rapidity $\eta$ at $p_{T}=5 \mathrm{GeV}$ and $\varphi=0, \pi / 4$.

Energy loss due to the synchrotron radiation has a very nontrivial azimuthal angle and rapidity dependence that comes from the corresponding dependence of the $\chi$ parameter (98). As can be seen in Figure 8(c), energy loss has a minimum at $\varphi=\pi / 2$ that corresponds to quark's transverse momentum $\mathbf{p}_{\perp}$ being parallel (or antiparallel) to the field direction. It has a maximum at $\varphi=0, \pi$ when $\mathbf{p}_{\perp}$ is perpendicular to the field direction and thus lying in the reaction plane. It is obvious from (98) that at midrapidity $\eta=0$ the azimuthal angle dependence is much stronger pronounced than in the forward/backward direction. Let me emphasize that the energy loss (96) divided by $m^{2}$, that is, $d \varepsilon /\left(d l \mathrm{~m}^{2}\right)$ scales with $\chi$. In turn, $\chi$ is a function of magnetic field, quark mass, rapidity, and azimuthal angle. Therefore, all the features seen in Figure 8 follow from this scaling behavior.

3.2. Azimuthal Asymmetry of Gluon Spectrum. In magnetic field gluon spectrum is azimuthally asymmetric. It is customary to describe this asymmetry by Fourier coefficients of intensity defined as

$$
\begin{gathered}
I(\varphi)=\bar{I}\left(1+2 \sum_{n=1}^{\infty} v_{n} \cos (n \varphi)\right), \\
v_{n}=\frac{1}{2 \pi \bar{I}} \int_{-\pi}^{\pi} I(\varphi) \cos (n \varphi) d \varphi .
\end{gathered}
$$

Azimuthal averaged intensity is $\bar{I}=\int_{-\pi}^{\pi} I(\varphi) d \varphi / 2 \pi$. In strong fields $\chi \gg 1$, and we can write

$$
\begin{aligned}
I(\varphi) & =0.37 \alpha_{s} C_{F} m^{2} \chi^{2 / 3} \\
& =0.37 \alpha_{s} C_{F}\left(e B p_{\perp}\right)^{2 / 3}\left(\sinh ^{2} \eta+\cos ^{2} \varphi\right)^{1 / 3} .
\end{aligned}
$$

We have

$$
\begin{aligned}
v_{n} & =\frac{\int_{-\pi}^{\pi} I(\varphi) \cos (n \varphi) d \varphi}{\int_{-\pi}^{\pi} I(\varphi) d \varphi} \\
& =\frac{\int_{-\pi}^{\pi}\left(\sinh ^{2} \eta+\cos ^{2} \varphi\right)^{1 / 3} \cos (n \varphi) d \varphi}{\int_{-\pi}^{\pi}\left(\sinh ^{2} \eta+\cos ^{2} \varphi\right)^{1 / 3} d \varphi} .
\end{aligned}
$$


At $\eta=0$ the Fourier coefficients $w_{n}$ can be calculated analytically using formula 3.631.9 of [34] as follows:

$$
\begin{gathered}
v_{n}=\frac{B(4 / 3,4 / 3)}{B((4 / 3)+(n / 2),(4 / 3)-(n / 2))}, \quad \text { if } n \in \text { even; } \\
v_{n}=0, \quad \text { if } n \in \text { odd, }
\end{gathered}
$$

where $B$ is Euler's beta function. The corresponding numerically values of the lowest harmonics are $v_{2}=0.25, v_{4}=$ $-0.071, v_{6}=0.036, v_{8}=-0.022$, and $v_{10}=0.015$.

3.3. Polarization of Light Quarks. Synchrotron radiation leads to polarization of electrically charged fermions, this is known as the Sokolov-Ternov effect [46]. It was applied to heavyion collisions in [21]. Unlike energy loss that I discussed so far, this is a purely quantum effect. It arises because the probability of the spin-flip transition depends on the orientation of the quark spin with respect to the direction of the magnetic field and on the sign of fermion's electric charge. The spin-flip probability per unit time reads [46]

$$
\begin{aligned}
w= & \frac{5 \sqrt{3} \alpha_{s} C_{F}}{16} \frac{\hbar^{2}}{m^{2}}\left(\frac{\varepsilon}{m}\right)^{5}\left(\frac{Z_{q} e|\mathbf{v} \times \mathbf{B}|}{\varepsilon}\right)^{3} \\
& \times\left(1-\frac{2}{9}(\zeta \cdot \mathbf{v})^{2}-\frac{8 \sqrt{3}}{15} \operatorname{sign}\left(e_{q}\right)(\zeta \cdot \widehat{\mathbf{z}})\right),
\end{aligned}
$$

where $\zeta$ is a unit axial vector that coincides with the direction of quark spin in its rest frame and $\mathbf{v}=\mathbf{p} / \varepsilon$ is the initial fermion velocity.

The nature of this spin-flip transition is transparent in the nonrelativistic case, where it is induced by the interaction Hamiltonian [49] as follows:

$$
\mathscr{H}=-\boldsymbol{\mu} \cdot \mathbf{B}=-\left(\frac{g e Z_{q} \hbar}{2 m}\right) \mathbf{s} \cdot \mathbf{B} .
$$

It is seen that negatively charged quarks and antiquarks (e.g., $\bar{u}$ and $d$ ) tend to align against the field, while the positively charged ones (e.g., $u$ and $\bar{d}$ ) align parallel to the field.

Let $n_{\uparrow(\downarrow)}$ be the number of fermions or antifermions with given momentum and spin direction parallel (antiparallel) to the field produced in a given event. At initial time $t=t_{0}$ the spinasymmetry defined as

$$
A=\frac{n_{\uparrow}-n_{\downarrow}}{n_{\uparrow}+n_{\downarrow}}
$$

vanishes. Equation (103) implies that at later times, a beam of nonpolarized fermions develops a finite asymmetry given by [46]

$$
A=\frac{8 \sqrt{3}}{15}\left(1-e^{-\left(t-t_{0}\right) / \tau}\right)
$$

where

$$
\tau=\frac{8}{5 \sqrt{3} m \alpha_{s} C_{F}}\left(\frac{m}{\varepsilon}\right)^{2}\left(\frac{m^{2}}{Z_{q} e|\mathbf{v} \times \mathbf{B}|}\right)^{3}
$$

is the characteristic time over which the maximal possible asymmetry is achieved. This time is extremely small on the scale of $t_{0}$. For example, it takes only $\tau=6 \cdot 10^{-8} \mathrm{fm}$ for a $d$ quark of momentum $p_{\perp}=1 \mathrm{GeV}$ at $\eta=\varphi=$ 0 at RHIC to achieve the maximal asymmetry of $A_{m}=$ $8 / 5 \sqrt{3}=92 \%$. Therefore, quarks and antiquarks are polarized almost instantaneously after being released from their wave functions. However, subsequent interaction with QGP and fragmentation washes out the polarization of quarks.

A more sensitive probe are leptons weakly interacting with QGP and not undergoing a fragmentation process. Thus, their polarization can present a direct experimental evidence for the existence and strength of magnetic field. In case of muons we can estimate $\tau$ by replacing $\alpha_{s} C_{F} \rightarrow \alpha_{\mathrm{em}}$. For muons we get $\tau=0.3 \mathrm{fm} / \mathrm{c}$, which is still much smaller than magnetic field life-time. Observation of such a lepton polarization asymmetry is perhaps the most definitive proof of existence of the strong magnetic field at early times after a heavy-ion collision regardless of its later time-dependence.

In summary, energy loss per unit length for a light quark with $p_{T}=15 \mathrm{GeV}$ is about $0.27 \mathrm{GeV} / \mathrm{fm}$ at RHIC and $1.7 \mathrm{GeV} / \mathrm{fm}$ at LHC, which is comparable to the losses due to interaction with the plasma. Thus, the synchrotron radiation alone is able to account for quenching of jets at LHC with $p_{\perp}$ as large as $20 \mathrm{GeV}$. Synchrotron radiation is definetely one of missing pieces in the puzzle of the jet energy loss in heavyion collisions. Quarks and leptons are expected to be strongly polarized in plasma in the direction parallel or anti-parallel to the magnetic field depending on the sign of their electric charge.

\section{Photon Decay}

In this section I consider pair production by photon in external magnetic field [55], which is a cross-channel of synchrotron radiation discussed in the previous section. Specifically, we are interested to determine photon decay rate $w$ in the process $\gamma B \rightarrow f \bar{f} B$, where $f$ stands for a charged fermion, as a function of photon's transverse momentum $k_{T}$, rapidity $\eta$, and azimuthal angle $\varphi$. Origin of these photons in heavy-ion collisions will not be of interest to us in this section.

Characteristic frequency of a fermion of species $a$ of mass $m_{a}$ and charge $z_{a} e$ ( $e$ is the absolute value of electron charge) moving in external magnetic field $B$ (in a plane perpendicular to the field direction) is

$$
\hbar \omega_{B}=\frac{z_{a} e B}{\varepsilon}
$$

where $\varepsilon$ is the fermion energy. Here-in the spirit of the adiabatic approximation $-B$ is a slow function of time. Calculation of the photon decay probability significantly simplifies if motion of electron is quasiclassical; that is, quantization of fermion motion in the magnetic field can be neglected. This condition is fulfilled if $\hbar \omega_{B} \ll \varepsilon$. This implies that

$$
\varepsilon \gg \sqrt{z e B} \text {. }
$$

For RHIC it is equivalent to $\varepsilon \gg m_{\pi}$, for LHC $\varepsilon \gg 4 m_{\pi}$. 
Photon decay rate was calculated in [45] and, using the quasi-classical method, in [56]. It reads

$$
w=-\sum_{a} \frac{\alpha_{\mathrm{em}} z_{a}^{3} e B}{m_{a} \varkappa_{a}} \int_{\left(4 / \varkappa_{a}\right)^{2 / 3}}^{\infty} \frac{2\left(x^{3 / 2}+1 / \varkappa_{a}\right) \mathrm{Ai}^{\prime}(x)}{x^{11 / 4}\left(x^{3 / 2}-4 / \varkappa_{a}\right)^{3 / 2}},
$$

where summation is over fermion species and the invariant parameter $\mathcal{u}$ is defined as

$$
\varkappa_{a}^{2}=-\frac{\alpha_{\mathrm{em}} z_{a}^{2} \hbar^{3}}{m_{a}^{6}}\left(F_{\mu \nu} k^{\nu}\right)^{2}=\frac{\alpha_{\mathrm{em}} z_{a}^{2} \hbar^{3}}{m_{a}^{6}}(\mathbf{k} \times \mathbf{B})^{2},
$$

with the initial photon 4-momentum $k^{\mu}=(\hbar \omega, \mathbf{k})$. With notations of Figure 7, $\mathbf{k}=k_{z} \widehat{\mathbf{z}}+k_{\perp}(\widehat{\mathbf{x}} \cos \varphi+\widehat{\mathbf{y}} \sin \varphi)$, where $k_{\perp}=|\mathbf{k}| \sin \theta=\hbar \omega \sin \theta$. Thus, $(\mathbf{B} \times \mathbf{k})^{2}=B^{2}\left(k_{z}^{2}+k_{\perp}^{2} \cos ^{2} \varphi\right)$. Employing $\hbar \omega=k_{\perp} \cosh \eta$ and $k_{z}=k_{\perp} \sinh \eta$, we write

$$
\varkappa_{a}=\frac{\hbar\left(z_{a} e B\right)}{m_{a}^{3}} k_{\perp} \sqrt{\sinh ^{2} \eta+\cos ^{2} \varphi} .
$$

Plotted in Figure 9 is the photon decay rate (110) for RHIC and LHC. The survival probability of photons in magnetic field is $P=1-w \Delta t$, where $\Delta t$ is the time spent by a photon in plasma. Estimating $\Delta t=10 \mathrm{fm}$ we determine that photon survives with probability $P_{\text {RHIC }} \approx 97 \%$ at RHIC, while only $P_{\mathrm{LHC}} \approx 80 \%$ at LHC. Such strong depletion can certainly be observed in heavy-ion collisions at LHC.

Azimuthal distribution of the decay rate of photons at LHC is azimuthally asymmetric as can be seen in Figure 10 [55]. The strongest suppression is in the $B$ field direction, that is, in the direction orthogonal to the reaction plane. At $\eta \gtrsim 1$ the $\varphi$ dependence of $\varkappa_{a}$ is very weak which is reflected in nearly symmetric azimuthal shape of the dashed line in Figure 10.

To quantify the azimuthal asymmetry it is customary to expand the decay rate in Fourier series with respect to the azimuthal angle. Noting that $w$ is an even function of $\varphi$, we have

$$
\begin{aligned}
& w(\varphi)=\frac{1}{2} w_{0}+\sum_{n=1}^{\infty} w_{n} \cos (n \varphi), \\
& w_{n}=\frac{1}{\pi} \int_{-\pi}^{\pi} w(\varphi) \cos (n \varphi) d \varphi .
\end{aligned}
$$

In strong fields $\varkappa_{a} \gg 1$. For example, for $\gamma \rightarrow \mu^{+} \mu^{-}$at RHIC at $\varphi=\eta=0$ and $k_{T}=1 \mathrm{GeV}$ we get $\varkappa_{\mu}=19$. Therefore, we can expand the rate (110) at large $\varkappa_{a}$ as [45]

$$
\begin{aligned}
w & \approx \frac{3^{1 / 6} 5 \Gamma^{2}(2 / 3)}{2^{4 / 3} 7 \pi^{1 / 2} \Gamma(7 / 6)} \sum_{a} \frac{\alpha_{\mathrm{em}} e B z_{a}^{3}}{m_{a} \varkappa_{a}^{1 / 3}} \\
& \equiv \frac{A}{\left(\sinh ^{2} \eta+\cos ^{2} \varphi\right)^{1 / 6}}, \quad \varkappa_{a} \gg 1 .
\end{aligned}
$$

At $\eta=0$ the Fourier coefficients $w_{n}$ can be calculated analytically using formula 3.631.9 of [34]

$$
\begin{gathered}
w_{2 k}=\frac{32^{1 / 3} A}{B((5 / 6)+k,(5 / 6)-k)}, \\
w_{2 k+1}=0, \quad k=0,1,2, \ldots,
\end{gathered}
$$

where $B$ is the Euler's beta function and $A$ is defined in (114), Substituting these expressions into (113) we find

$$
w=\frac{1}{2} w_{0}\left[1-\sum_{k=1}^{\infty} \frac{\sqrt{\pi} \Gamma(-1 / 6)}{2^{2 / 3} B((5 / 6)+k,(5 / 6)-k)} \cos (2 k \varphi)\right] .
$$

The first few terms in this expansion read

$$
w=\frac{1}{2} w_{0}\left(1-\frac{2}{5} \cos (2 \phi)+\frac{14}{55} \cos (4 \phi)-\cdots\right),
$$

What is measured experimentally is not the decay rate, but rather the photon spectrum. This spectrum is modified by the survival probability $P$ which is obviously azimuthally asymmetric. To quantify this asymmetry, we write using (113)

$$
\begin{gathered}
P=\bar{P}\left(1+\sum_{k=1}^{\infty} v_{2 k} \cos (2 \varphi k)\right), \\
v_{2 k}=-\frac{1-\bar{P}}{\bar{P}} \frac{2 w_{2 k}}{w_{0}},
\end{gathered}
$$

where $\bar{P}=\langle 1-w \Delta t\rangle_{\varphi}=1-w_{0} \Delta t$ is the survival probability averaged over the azimuthal angle. Since $w_{0} \Delta t \ll 1$, as can be seen in Figure 9, we can estimate using (114) and (115)

$$
\begin{aligned}
v_{2 k} & \approx-\frac{2 w_{2 k}}{w_{0}} w_{0} \Delta t \\
& =-\frac{2 w_{2 k}}{w_{0}} \Delta t \frac{56^{2 / 3} \Gamma(2 / 3)}{7 \pi} \sum_{a} \frac{\alpha_{\mathrm{em}}(e B)^{2 / 3} z_{a}^{8 / 3}}{\left(k_{T}\right)^{1 / 3}} .
\end{aligned}
$$

In particular, the "elliptic flow" coefficient is [55]

$$
v_{2}=\Delta t \frac{26^{2 / 3} \Gamma(2 / 3)}{7 \pi} \sum_{a} \frac{\alpha_{\mathrm{em}}(e B)^{2 / 3} z_{a}^{8 / 3}}{\left(k_{T}\right)^{1 / 3}} .
$$

For example, at $k_{T}=1 \mathrm{GeV}$ and $\Delta t \sim 10 \mathrm{fm} / \mathrm{c}$ one expects $v_{2} \simeq 2 \%$ at RHIC and $v_{2} \simeq 14 \%$ at LHC only due to the presence of magnetic field. We see that decay of photons in external magnetic field significantly contributes to the photon asymmetry in heavy-ion collisions along with other possible effects.

In summary, I calculated photon pair-production rate in external magnetic field created in off-central heavy-ion collisions. Photon decay leads to depletion of the photon yield by a few percent at RHIC and by as much as $20 \%$ at the LHC. The decay rate depends on the rapidity and azimuthal angle. At midrapidity the azimuthal asymmetry of the decay rate translates into asymmetric photon yield and contributes to the "elliptic flow." Let me also quote a known result that photons polarized parallel to the field are $3 / 2$ times more likely to decay than those polarized transversely [45]. Therefore, polarization of the final photon spectrum perpendicular to the field is a signature of existence of strong magnetic field. Finally, photon decay necessarily leads to enhancement of dilepton yield. 


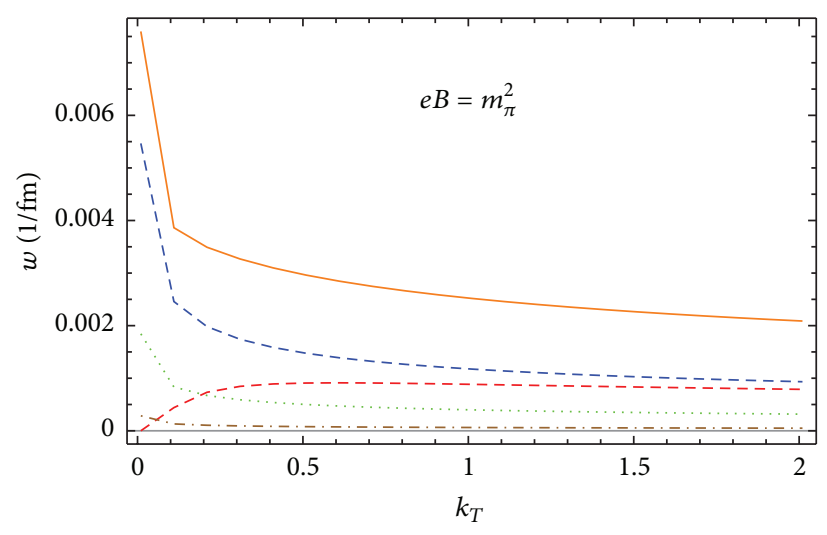

(a)

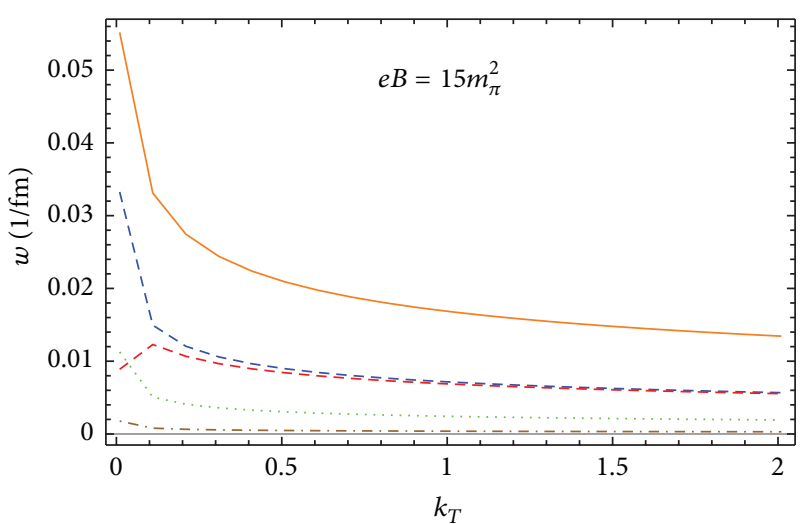

(b)

FIGURE 9: Decay rate of photons moving in reaction plane in magnetic field as a function of transverse momentum $k_{T}$ : (a) at RHIC, (b) at LHC. Broken lines from bottom to top give contributions of $\gamma \rightarrow d \bar{d}, \gamma \rightarrow u \bar{u}, \gamma \rightarrow \mu^{+} \mu^{-}$, and $\gamma \rightarrow e^{+} e^{-}$channels. Upper solid line is the total rate.

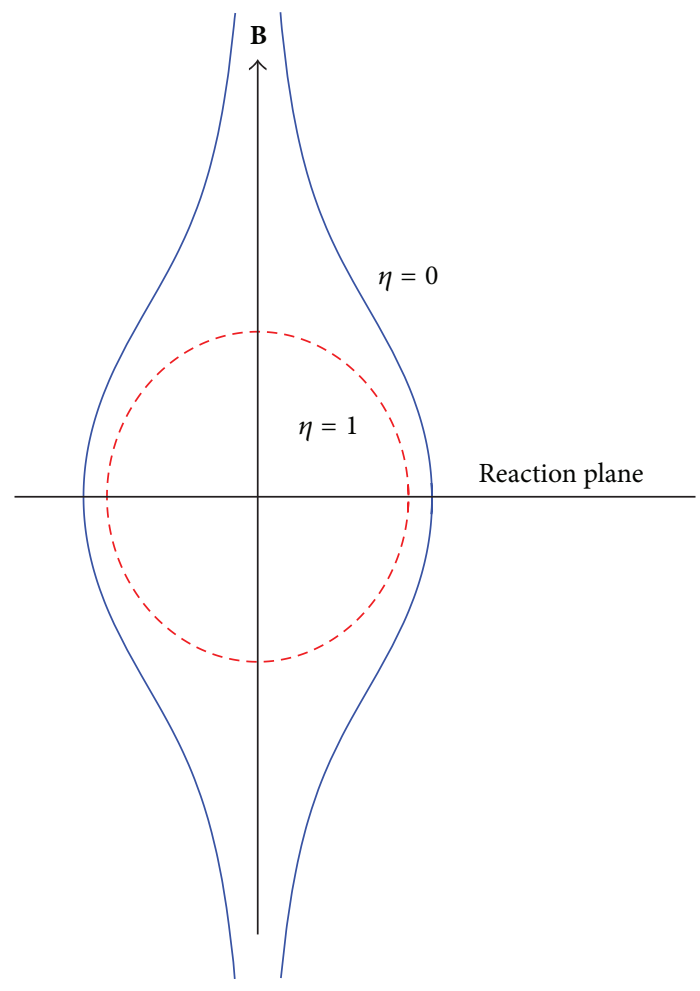

FIGURE 10: Azimuthal distribution of the decay rate of photons at different rapidities at LHC. Only contribution of the $\gamma \rightarrow e^{+} e^{-}$ channel is shown. Note that the rate $w$ vanishes at $\eta=0$ and $\varphi=\pi / 2$, that is, in the direction of $\mathbf{B}$. However, it happens at so small values of $|\varphi-\pi / 2|$ that it is beyond the experimental resolution and is not shown in the figure.

\section{Quarkonium Dissociation in Magnetic Field}

5.1. Effects of Magnetic Field on Quarkonium. Strong magnetic field created in heavy-ion collisions generates a number of remarkable effects on quarkonium production, some of which I will describe in this section. Magnetic field can be treated as static if the distance $\lambda$ over which it significantly varies is much larger than the quarkonium radius. If $\Delta t$ is magnetic field life-time, then $\lambda \sim c \Delta t$. For a quarkonium with binding energy $\varepsilon_{b}$ and radius $\alpha_{s} / \varepsilon_{b}$, the quasi-static approximation applies when $\varepsilon_{b} \lambda / \alpha_{s} \gg 1$. Estimating conservatively $\lambda \sim 2 \mathrm{fm}$ we get for $J / \psi: \varepsilon_{b} \lambda / \alpha_{s} \approx 23$, which is comfortably large to justify the quasi-static approximation, where I assumed that $\varepsilon_{b}$ is given by its vacuum value. As temperature $T$ increases $\varepsilon_{b}$ drops. Temperature dependence of $\varepsilon_{b}$ is model dependent; however, it is certain that eventually it vanishes at some finite temperature $T_{0}$. Therefore, only in the close vicinity of $T_{0}$, that is, at very small binding energies, the quasi-static approximation is not applicable. I thus rely on the quasi-static approximation to calculate $J / \psi$ dissociation $[57,58]$.

Magnetic field has a three-fold effect on quarkonium.

(1) Lorentz ionization. Consider quarkonium traveling with constant velocity in magnetic field in the laboratory frame. Boosting to the quarkonium comoving frame, we find mutually orthogonal electric and magnetic fields given by (121a), (121b), and (122). In the presence of an electric field quark and antiquark have a finite probability to tunnel through the potential barrier thereby causing quarkonium dissociation. In atomic physics such a process is referred to as Lorentz ionization. In the nonrelativistic approximation, the tunneling probability is of order unity when the electric field $E$ in the comoving frame satisfies $e E \gtrsim m^{1 / 2} \varepsilon_{b}^{3 / 2}$ (for weakly bound states), where $m$ is quark mass; see (144). This effect causes a significant increase in quarkonium dissociation rate; numerical calculation for $J / \psi$ is shown in Figure 13.

(2) Zeeman effect. Energy of a quarkonium state depends on spin $S$, orbital angular momentum $L$, and total angular momentum $J$. In a magnetic field these states split; the splitting energy in a weak field is $\Delta M=$ $\left(e B_{0} / 2 m\right) g J_{z}$, where $J_{z}=-J,-J+1, \ldots, J$ is projection of the total angular momentum on the direction of magnetic field, $m$ is quark mass, and $g$ is Landé factor depending on $J, L$, and $S$ in a well-known way; see, for 
example, [59]. For example, $J / \psi$ with $S=1, L=0$, and $J=1(g \approx 2)$ splits into three states with $J_{z}=$ $\pm 1,0$ and with mass difference $\Delta M=0.15 \mathrm{GeV}$, where we used $e B_{0}=15 m_{\pi}^{2}$. Thus, the Zeeman effect leads to the emergence of new quarkonium states in plasma.

(3) Distortion of the quarkonium potential in magnetic field. This effect arises in higher-order perturbation theory and becomes important at field strengths of order $B \sim 3 \pi m^{2} / e^{3}[60]$. This is $3 \pi / \alpha$ times stronger than the critical Schwinger's field. Therefore, this effect can be neglected at the present collider energies.

Some of the notational definitions used in this section: $\mathbf{V}$ and $\mathbf{P}$, are velocity and momentum of quarkonium in the lab frame; $M$ is its mass; $\mathbf{p}$ is the momentum of quark or antiquark in the comoving frame; $m$ is its mass; $\mathbf{B}_{0}$ is the magnetic field in the lab frame, $\mathbf{E}$ and $\mathbf{B}$ are electric and magnetic fields in the comoving frame; $\gamma_{L}$ is the quarkonium Lorentz factor; and $\gamma$ is a parameter defined in (139). I use Gauss units throughout the section; note that expressions $e B, e E$, and $e B_{0}$ are the same in Gauss and Lorentz-Heaviside units.

5.2. Lorentz Ionization: Physical Picture. In this section I focus on Lorentz ionization, which is an important mechanism of $J / \psi$ suppression in heavy-ion collisions $[57,58]$. Before we proceed to analytical calculations it is worthwhile to discuss the physics picture in more detail in two reference frames: the quarkonium proper frame and the lab frame. In the quarkonium proper frame the potential energy of, say, antiquark (with $e<0$ ) is a sum of its potential energy in the binding potential and its energy in the electric field $-e E x$, where $x$ is the electric field direction; see Figure 11. Since $|e| E x$ becomes large and negative at large and negative $x$ (far away from the bound state) and because the quarkonium potential has finite radius, this region opens up for the motion of the antiquark. Thus there is a quantum mechanical probability to tunnel through the potential barrier formed on one side by the vanishing quarkonium potential and on the other by increasing absolute value of the antiquark energy in electric field. Of course the total energy of the antiquark (not counting its mass) is negative after tunneling. However, its kinetic energy grows proportionally to $e E x$ as it goes away. By picking up a light quark out of vacuum it can hadronize into a $D$ meson.

If we now go to the reference frame where $E=0$ and there is only magnetic field $B$ (we can always do so since $E<$ $B)$, then the entire process looks quite different. An energetic quarkonium travels in external magnetic field and decays into quark-antiquark pair that can later hadronize into $D$-mesons. This happens in spite of the fact that $J / \psi$ mass is smaller than masses of two $D$-mesons due to additional momentum $e \mathbf{A}$ supplied by the magnetic field. Similarly a photon can decay into electron-positron pair in external magnetic field.

\subsection{Quarkonium Ionization Rate}

5.3.1. Comoving Frame. Consider a quarkonium traveling with velocity $\mathbf{V}$ in constant magnetic field $\mathbf{B}_{0}$. Let $\mathbf{B}$ and $\mathbf{E}$

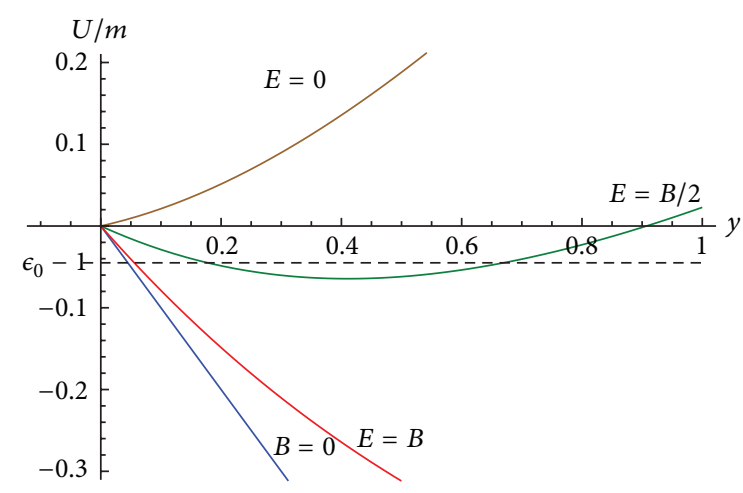

FIGURE 11: Effective potential $U(x)=\sqrt{m^{2}+\left(p_{z}+e B x\right)^{2}+p_{x}^{2}}-$ $e E x-\sqrt{m^{2}+p_{x}^{2}+p_{x}^{2}}$ for $p_{x}=0, p_{z}=m / 6$, and $B=m$ (except the blue line where $B=0$ ). The width of the potential barrier decreases with $E$ and increases with $B .1-\epsilon_{0}$ corresponds to the binding energy in units of $m$.

be magnetic and electric fields in the comoving frame, and let subscripts $\|$ and $\perp$ denote field components parallel and perpendicular to $\mathbf{V}$ correspondingly. Then,

$$
\begin{array}{r}
E_{\|}=0, \quad \mathbf{E}_{\perp}=\gamma_{L} \mathbf{V} \times \mathbf{B}_{0}, \\
B_{\|}=\frac{\mathbf{B}_{0} \cdot \mathbf{V}}{V}, \quad \mathbf{B}_{\perp}=\gamma_{L} \frac{\left(\mathbf{V} \times \mathbf{B}_{0}\right) \times \mathbf{V}}{V^{2}},
\end{array}
$$

where $\gamma_{L}=\left(1-V^{2}\right)^{-1 / 2}$. Clearly, in the comoving frame $\mathbf{B}$. $\mathbf{E}=0$. If quarkonium travels at angle $\phi$ with respect to the magnetic field in the laboratory frame, then

$$
B=B_{0} \sqrt{\cos ^{2} \phi\left(1-\gamma_{L}^{2}\right)+\gamma_{L}^{2}}, \quad E=B_{0} \gamma_{L} V \sin \phi .
$$

We choose $y$ and $x$ axes of the comoving frame such that $\mathbf{B}=B \widehat{\mathbf{y}}$ and $\mathbf{E}=E \widehat{\mathbf{x}}$. A convenient gauge choice is $\mathbf{A}=-B x \widehat{\mathbf{z}}$ and $\varphi=-E x$. For a future reference we also define a useful dimensionless parameter $\rho$ [61]:

$$
\rho=\frac{E}{B}=\frac{\gamma_{L} V \sin \phi}{\sqrt{\cos ^{2} \phi\left(1-\gamma_{L}^{2}\right)+\gamma_{L}^{2}}} .
$$

Note that (i) $0 \leq \rho \leq 1$ because $B^{2}-E^{2}=B_{0}^{2} \geq 0$ and (ii) when quarkonium moves perpendicularly to the magnetic field $\mathbf{B}_{0}$, $\rho=V$.

5.3.2. WKB Method. I assume that the force binding $q$ and $\bar{q}$ into quarkonium as a short-range one, that is, $\left(M \varepsilon_{b}\right)^{1 / 2} R \ll 1$, where $\varepsilon_{b}$ and $M$ are binding energy and mass of quarkonium, respectively, and $R$ is the radius of the nuclear force given by $R \approx\left(\alpha_{s} / \sigma\right)^{1 / 2}$, where $\sigma=1 \mathrm{GeV} / \mathrm{fm}$ is the string tension. For example, the binding energy of $c$ and $\bar{c}$ in $J / \psi$ in vacuum is $\varepsilon_{b}=0.64 \mathrm{GeV} \ll M / R^{2}=M \sigma / \alpha_{s} \approx 3 \mathrm{GeV}$. This approximation is even better at finite temperature on account of $\varepsilon_{b}$ decrease. Regarding $J / \psi$ as being bound by a shortrange force enables us to calculate the dissociation probability 
$w$ with exponential accuracy $w \approx e^{-f}$, independently of the precise form of the quarkonium wave function. This is especially important, since solutions of the relativistic twobody problem for quarkonium are not readily available.

It is natural to study quarkonium ionization in the comoving frame [57]. As explained in the Introduction, ionization is quantum tunneling through the potential barrier caused by the electric field $\mathbf{E}$. In this subsection I employ the quasiclassical WKB approximation to calculate the quarkonium decay probability $w$. For the gauge choice specified in Section 5.3.1, quark energy $\varepsilon_{0}\left(\varepsilon_{0}<m\right)$ in electromagnetic field can be written as

$$
\begin{aligned}
\varepsilon_{0} & =\sqrt{m^{2}+(\mathbf{p}-e \mathbf{A})^{2}}+e \varphi \\
& =\sqrt{m^{2}+\left(p_{z}+e B x\right)^{2}+p_{x}^{2}+p_{y}^{2}}-e E x .
\end{aligned}
$$

In terms of $\varepsilon_{0}$, quarkonium binding energy is $\varepsilon_{b}=m-\varepsilon_{0}$. To simplify notations, we will set $p_{x}=0$, because the quark moves constant momentum along the direction of magnetic field.

The effective potential $U(x)=\varepsilon_{0}(x)-\sqrt{m^{2}+\mathbf{p}^{2}}$ corresponding to (124) is plotted in Figure 11. We can see that the tunneling probability is finite only if $E>0$. It is largest when $B=0$. It has been already noted before in [61-63] that the effect of the magnetic field is to stabilize the bound state. In spite of the linearly rising potential (at $B>E$ ) tunneling probability is finite as the result of rearrangement of the QED vacuum in electric field.

Ionization probability of quarkonium equals its tunneling probability through the potential barrier. The later is given by the transmission coefficient

$$
w=e^{-2 \int_{0}^{y_{1}} \sqrt{-p_{y}^{2}} d y} \equiv e^{-f} .
$$

In the nonrelativistic approximation one can also calculate the preexponential factor, which appears due to the deviation of the quark wave function from the quasi-classical approximation. This is discussed later in Section 5.5.2. We now proceed with the calculation of function $f$. Since $B>E$ (125) can be written as [57]

$$
p_{x}^{2}=-e^{2}\left(B^{2}-E^{2}\right)\left(x-x_{1}\right)\left(x-x_{2}\right),
$$

where

$$
x_{1,2}=\frac{\varepsilon_{0} E-p_{z} B \mp \sqrt{\left(\varepsilon_{0} E-p_{z} B\right)^{2}-\left(B^{2}-E^{2}\right)\left(-\varepsilon_{0}^{2}+m^{2}+p_{z}^{2}\right)}}{e\left(B^{2}-E^{2}\right)} .
$$

Define dimensionless variables $\epsilon_{0}=\varepsilon_{0} / m$ and $q=p_{z} / m$. Integration in (74) gives

$$
\begin{aligned}
\frac{f}{m^{2}}= & \frac{\sqrt{-\epsilon_{0}^{2}+1+q^{2}}\left(\epsilon_{0} E-q B\right)}{e\left(B^{2}-E^{2}\right)} \\
& -\frac{\left(\epsilon_{0} E-q B\right)^{2}-\left(B^{2}-E^{2}\right)\left(-\epsilon_{0}^{2}+1+q^{2}\right)}{e\left(B^{2}-E^{2}\right)^{3 / 2}} \\
& \times \ln \left\{\frac{\epsilon_{0} E-q B+\sqrt{\left(B^{2}-E^{2}\right)\left(-\epsilon_{0}^{2}+1+q^{2}\right)}}{\sqrt{\left(\epsilon_{0} E-q B\right)^{2}-\left(B^{2}-E^{2}\right)\left(\epsilon_{0}^{2}+1+q^{2}\right)}}\right\} .
\end{aligned}
$$

$w=e^{f}$ gives the corresponding ionization probabilities, at a given $q$. The largest probability corresponds to smallest $f$, which occurs at momentum $q_{m}$ determined by [62]

$$
\frac{\partial f\left(q_{m}\right)}{\partial q_{m}}=0
$$

Using (128) and parameter $\rho$ defined in (123), we find [57]

$$
\begin{aligned}
& \frac{\rho\left(\epsilon_{0}-\rho q_{m}\right)}{1-\rho^{2}} \ln \left\{\frac{\epsilon_{0} \rho-q_{m}+\sqrt{1-\rho^{2}} \sqrt{-\epsilon_{0}^{2}+1+q_{m}^{2}}}{\sqrt{\left(\epsilon_{0}-\rho q_{m}\right)^{2}-1+\rho^{2}}}\right\} \\
& =\frac{\sqrt{-\epsilon_{0}^{2}+1+q_{m}^{2}}}{\sqrt{1-\rho^{2}}} .
\end{aligned}
$$

This is an implicit equation for the extremal momentum $q_{m}=$ $q_{m}\left(\epsilon_{0}, \rho\right)$. Substituting $q_{m}$ into (128) one obtains $f=f\left(\epsilon_{0}, \rho\right)$, which by means of (125) yields the ionization probability. The quasi-classical approximation that we employed in this section is valid in as much as $f\left(q_{m}\right) \gg 1$.

In order to compare with the results obtained in [62] using the imaginary time method, we can rewrite (130) in terms of an auxiliary parameter $\tau_{0}$ as

$$
\begin{gathered}
\tau_{0}=\frac{\sqrt{1-\rho^{2}} \sqrt{-\epsilon_{0}^{2}+1+q_{m}^{2}}}{\rho\left(\epsilon_{0}-\rho q_{m}\right)}, \\
\frac{\tanh \tau_{0}}{\tau_{0}}=\rho \frac{\epsilon_{0}-\rho q_{m}}{\epsilon_{0} \rho-q_{m}} .
\end{gathered}
$$

Taking advantage of these equations, (128) can be cast into a more compact form as follows

$$
f_{m}=\frac{m^{2} \tau_{0} \rho}{e E \sqrt{1-\rho^{2}}}\left[1-\epsilon_{0}\left(\epsilon_{0}-q_{m} \rho\right)\right]
$$

where we denoted $f_{m}=f\left(q_{m}\right)$. This agrees with results of [62]. 
5.3.3. Special Case: Crossed Fields. An important limiting case is crossed fields $E=B$. Since also $\mathbf{E} \perp \mathbf{B}$ (see Section 5.3.1), both field invariants vanish. Nevertheless, quarkonium ionization probability is finite [62]. This limit is obtained by taking $\rho \rightarrow 1$ in the equations from the previous section. Employing (131a) and (131b) we get the following condition for extremum:

$$
\epsilon_{0}^{2}-1+2 q_{m}^{2}-3 \epsilon_{0} q_{m}=0
$$

with the solution

$$
q_{m}=\frac{1}{4}\left(3 \epsilon_{0}-\sqrt{\epsilon_{0}^{2}+8}\right) .
$$

Substituting into (132) produces

$$
f_{m}=\frac{2}{3} \frac{m^{2}}{e E} \frac{\left(-\epsilon_{0}^{2}+1+q_{m}^{2}\right)^{3 / 2}}{\epsilon_{0}-q_{m}} .
$$

5.4. Nonrelativistic Approximation. A very useful approximation of the relativistic formulas derived in the previous section is the nonrelativistic limit because (i) it provides a very good numerical estimate (see Figure 12), and (ii) it allows us to eliminate the parametric dependence in (128), (130) and write $f\left(q_{m}\right)$ explicitly in terms of $\rho$ and $\epsilon_{0}$, and (iii) spin effects can be accounted for $[57,58]$.

5.4.1. Arbitrary Binding. Motion of a particle can be treated non-relativistically if its momentum is much less than its mass. In such a case $\varepsilon_{0} \approx m$ or $\varepsilon_{b}=m-\varepsilon_{0} \ll m$. Additionally, motion of a charged particle in electromagnetic field is non-relativistic if $E \ll B$. Indeed, the average velocity of a nonrelativistic particle is of order $v \sim E / B=\rho$. Thus, the non-relativistic limit is obtained by taking the limits $\epsilon_{b}=$ $\varepsilon_{b} / m \ll 1$ and $\rho \ll 1$. In these limits the extremum conditions (131a), (131b) reduce to

$$
\begin{gathered}
\tau_{0}=\frac{\sqrt{2 \epsilon_{b}+q_{m}^{2}}}{\rho}, \\
\frac{\tanh \tau_{0}}{\tau_{0}}=\frac{\rho}{\rho-q_{m}} .
\end{gathered}
$$

Out of two solution to (136a) we pick the following one:

$$
q_{m}=-\sqrt{\tau_{0}^{2} \rho^{2}-2 \epsilon_{b}}
$$

The sign of $q_{m}$ is fixed using (136b) by noticing that $\tanh \tau_{0} / \tau_{0}<1$. Eliminating $q_{m}$ gives

$$
\tau_{0}^{2}-\left(\tau_{0} \operatorname{coth} \tau_{0}-1\right)^{2}=\gamma^{2}
$$

where

$$
\gamma=\frac{\sqrt{2 \epsilon_{b}}}{\rho}
$$

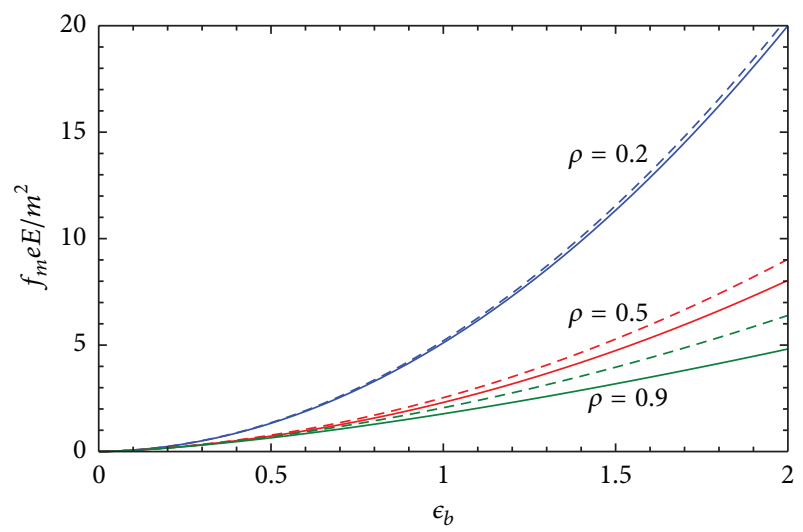

Figure 12: Dimensionless function $f_{m} e E / m^{2}$ versus $\epsilon_{b}$ for different values of $\rho$. The solid line is the full relativistic calculation, the dashed line is the nonrelativistic approximation. $J / \psi$ binding energy in vacuum corresponds to $\epsilon_{b}=0.68$

where $\gamma$ is analogous to the adiabaticity parameter of Keldysh [64]. Taking the non-relativistic limit of (132) and using (137) yields

$$
f_{m}=\frac{2 m^{2}\left(2 \epsilon_{b}\right)^{3 / 2}}{3 e E} g(\gamma),
$$

where $g(\gamma)$ is the Keldysh function [64]

$$
g(\gamma)=\frac{3 \tau_{0}}{2 \gamma}\left[1-\frac{1}{\gamma}\left(\frac{\tau_{0}^{2}}{\gamma^{2}}-1\right)^{1 / 2}\right] .
$$

In Figure 12 we show the dimensionless ratio $f_{m} e E / m^{2}$ as a function of the binding energy $\epsilon_{b}$ (in units of $m$ ) for several values of $\rho$. The vacuum binding energy of $J / \psi$ corresponds to $\epsilon_{b}=0.68$. We observe an excellent agreement between the full relativistic calculation and the non-relativistic approximation. At $\rho=0.9$ and $\epsilon_{b}=0.68$ the difference between the two lines is $10 \%$ and can be further improved by considering higher order corrections to $f_{m}$ [63].

5.4.2. Weak Binding. Of special interest is the limit of weak binding $\gamma \ll 1$; that is, $\sqrt{2 \epsilon_{b}} \ll \rho$. Expanding (138) at small $\gamma$ and $\tau_{0}$ yields

$$
\tau_{0}=\gamma\left(1+\frac{1}{18} \gamma^{2}\right)
$$

and substituting into (141) and subsequently into (140) yields

$$
f_{m}=\frac{2}{3} \frac{m^{2}}{e E}\left(2 \epsilon_{b}\right)^{3 / 2}
$$

Hence, the quarkonium dissociation probability reads [65]

$$
w=\exp \left\{-\frac{2}{3} \frac{\left(2 \epsilon_{b} m\right)^{3 / 2}}{m e E}\right\}
$$




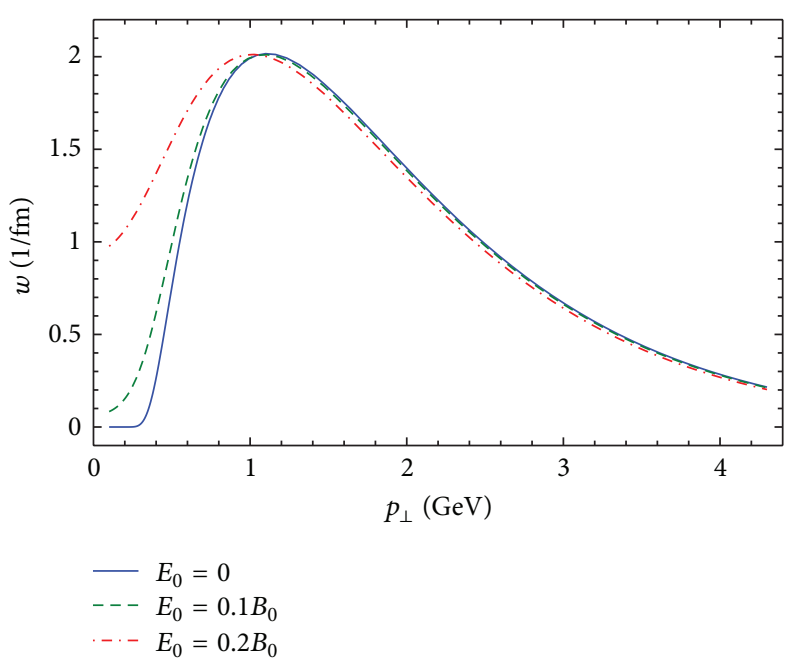

(a)

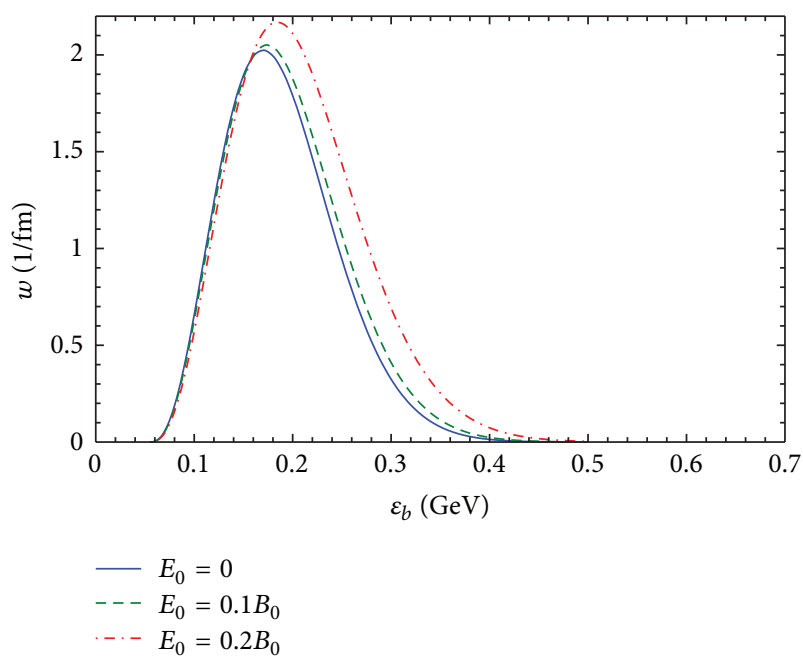

(b)

Figure 13: Dissociation rate of $J / \psi$ at $e B_{0}=15 m_{\pi}^{2}, \phi=\pi / 2$ (in the reaction plane), and $\eta=0$ (midrapidity) as a function of (a) $P_{\perp}$ at $\varepsilon_{b}=0.16 \mathrm{GeV}$ and (b) $\varepsilon_{b}$ at $P_{\perp}=1 \mathrm{GeV}$.

Since the quasi-classical approximation employed in this paper is valid if $f\left(q_{m}\right) \gg 1$, it follows that the binding energy must satisfy

$$
\frac{(e E)^{2 / 3}}{m^{1 / 3}} \ll \varepsilon_{b} \ll \rho^{2} m
$$

Note also that we work in the approximation of the shortrange binding potential meaning that $\sqrt{2 \epsilon_{b}} \ll 1 /(m R)$; see Section 5.1.

5.4.3. Strong Binding. In the limit $\gamma \gg 1,(138)$ and (141) imply that

$$
\tau_{0}=\frac{\gamma^{2}}{2}, \quad g(\gamma)=\frac{3 \gamma}{8} .
$$

Substituting (146) into (140), we derive

$$
f_{m}=\frac{\varepsilon_{b}^{2}}{e E} \frac{B}{E}
$$

Thus, quarkonium dissociation probability in the case of strong binding is

$$
w=\exp \left\{-\frac{\varepsilon_{b}^{2}}{e E} \frac{B}{E}\right\} .
$$

This formula is valid when

$$
\rho^{2} m, \quad \sqrt{e E \rho} \ll \varepsilon_{b} \ll \frac{1}{R} .
$$

5.4.4. Contribution of Quark Spin. So far I have neglected the contribution of quark spin. In order to take into account the effect of spin interaction with the external field, we can use squared Dirac equation for a bispinor $\psi$ as follows:

$$
\left[(\varepsilon-e \varphi)^{2}-(\mathbf{p}-e \mathbf{A})^{2}-m^{2}+e \boldsymbol{\Sigma} \cdot \mathbf{B}-i e \boldsymbol{\alpha} \cdot \mathbf{E}\right] \psi=0,
$$

where

$$
\Sigma=\left(\begin{array}{cc}
\sigma & 0 \\
0 & \sigma
\end{array}\right), \quad \boldsymbol{\alpha}=\left(\begin{array}{cc}
\sigma & 0 \\
0 & -\sigma
\end{array}\right) .
$$

Operators $\Sigma_{y}$ and $\alpha_{x}$ do not commute. Therefore, in order to apply the WKB method for calculation of the ionization probability one actually needs to square (150), which leads to a differential equation of the fourth order in derivatives. The problem becomes more tractable in the non-relativistic case and for crossed fields. Spin effects in crossed fields were discussed in [66].

With quark spin taken into account, the non-relativistic version of (124) becomes

$$
\frac{1}{2 m}\left[\left(p_{z}+e B x\right)^{2}+p_{x}^{2}\right]-e E x-\frac{\mu}{s} \mathbf{s} \cdot \mathbf{B}=-\varepsilon_{b},
$$

and hence

$$
p_{x}^{2}=2 m\left(-\varepsilon_{b}+\frac{\mu}{s} \mathbf{s} \cdot \mathbf{B}+e E x\right)-\left(p_{z}+e B x\right)^{2},
$$

where $\mu$ is the quark magnetic moment and $s$ is the projection of spin in the direction of the magnetic field. For a point quark, $\mu=\mu_{B}=e \hbar / 2 m c$. The effect of quark spin on quarkonium dissociation probability can be taken into account by replacing $\varepsilon_{b} \rightarrow \varepsilon_{b}^{\prime}=\varepsilon_{b}-(\mu / s) \mathbf{s} \cdot \mathbf{B}$ in formulas for $f_{m}$. With this replacement, all results of this section apply to a particle with spin. Note that effective binding energy $\varepsilon_{b}^{\prime}$ decreases if spin is parallel to the magnetic field and increases if it is antiparallel. In particular, in the case of weak binding

$$
w=\sum_{s= \pm 1 / 2} \exp \left\{-\frac{2}{3} \frac{\left(2 \varepsilon_{b} m+2 s e B\right)^{3 / 2}}{m e E}\right\} .
$$

Since the non-relativistic limit provides a good approximation of the full relativistic formulas, we will implement the quark spin dependence using the non-relativistic prescription $[57,58]$. 


\subsection{Effect of Electric Field Produced in the Lab Frame}

5.5.1. Origin of Electric Field in the Lab Frame. So far I have entirely neglected possible existence of electric field in the lab frame. This field, which we will be denoted by $\mathbf{E}_{0}$, can have two origins: (i) asymmetry of nucleon distributions in the colliding heavy ions (see Figure 3(b)) and (ii) chiral magnetic effect (CME) [5, 67-72], which has recently attracted a lot of attention. In a nutshell, if a metastable $P$ - and $C P$-odd bubble is induced by axial anomaly in the hot nuclear matter, then in the presence of external magnetic field $\mathbf{B}_{0}$ the bubble generates an electric field which is parallel to the magnetic one. According to [68] the value of the electric field $\mathbf{E}_{0}$ in the bubble is

$$
\mathbf{E}_{0}=-N_{c} \sum_{f} \frac{e_{f}^{2}}{4 \pi^{2}} \frac{\Theta}{N_{f}} \mathbf{B}_{0}=-\frac{2}{3} \frac{\alpha \Theta}{\pi} \mathbf{B}_{0},
$$

where the sum runs over quark flavors $f$ and it is assumed that only three lightest flavors contribute. The value of the $\Theta$-angle fluctuates from event to event. CME refers to the macroscopic manifestation of this effect-separation of electric charges with respect to the reaction plane. This effect is a possible explanation of experimentally observed charge asymmetry fluctuations [73-75].

No matter what is the origin of electric field in the lab frame, it averages to zero over an ensemble of events. We are interested to know the effect of this field on quarkonium dissociation - this is the problem we are turning to now [58].

5.5.2. Quarkonium Dissociation Rate. Ionization probability of quarkonium equals its tunneling probability through the potential barrier. In the $\mathrm{WKB}$ approximation the later is given by the transmission coefficient and was calculated in Section 5.3. In this method contribution of the quark spin can be easily taken into account. Another method of calculating the ionization probability, the imaginary time method [76-78], was employed in [61-63]. It also yielded in the non-relativistic approximation the preexponential factor that appears due to the deviation of the quark wave function from the quasi-classical approximation. Such a calculation requires matching quark wave function inside and outside the potential barrier [65]. Extension of this approach to the relativistic case is challenging due to analytical difficulties of the relativistic two-body problem. Fortunately, it was argued in Section 5.3, that the non-relativistic approximation provides a very good accuracy in the $\varepsilon_{b} \ll m$ region, which is relevant in the quarkiononium dissociation problem $[57,62]$.

Given the electromagnetic field in the laboratory frame $\mathbf{B}_{0}, \mathbf{E}_{0}$, the electromagnetic field $\mathbf{B}, \mathbf{E}$ in the comoving frame moving with velocity $\mathbf{V}$ is given by

$$
\begin{aligned}
& \mathbf{E}=E_{0}\left\{\gamma_{L}\left(\mathbf{b}_{0}+\rho_{0}^{-1} \mathbf{V} \times \mathbf{b}_{0}\right)-\left(\gamma_{L}-1\right) \mathbf{V} \frac{\mathbf{V} \cdot \mathbf{b}_{0}}{V^{2}}\right\}, \\
& \mathbf{B}=B_{0}\left\{\gamma_{L}\left(\mathbf{b}_{0}-\rho_{0} \mathbf{V} \times \mathbf{b}_{0}\right)-\left(\gamma_{L}-1\right) \mathbf{V} \frac{\mathbf{V} \cdot \mathbf{b}_{0}}{V^{2}}\right\},
\end{aligned}
$$

where $\mathbf{b}_{0}=\mathbf{B}_{0} / B_{0}$ is a unit vector in the magnetic field direction, $\rho_{0}=E_{0} / B_{0}=2 \alpha|\Theta| / 3 \pi$ (see (155)) and $\gamma_{L}=$ $1 / \sqrt{1-V^{2}}$. It follows from (156a) and (156b) that

$$
\begin{aligned}
& E=E_{0} \sqrt{1+\gamma_{L}^{2}\left(\mathbf{b}_{0} \times \mathbf{V}\right)^{2}\left(1+\rho_{0}^{-2}\right)}, \\
& B=B_{0} \sqrt{1+\gamma_{L}^{2}\left(\mathbf{b}_{0} \times \mathbf{V}\right)^{2}\left(1+\rho_{0}^{2}\right)} .
\end{aligned}
$$

Using (157a) and (157b), we find that the angle $\theta$ between the electric and magnetic field in the comoving frame is

$$
\begin{aligned}
\cos \theta & =\frac{\mathbf{E} \cdot \mathbf{B}}{E B} \\
& =\frac{1}{\sqrt{\left[1+\gamma_{L}^{2}\left(\mathbf{b}_{0} \times \mathbf{V}\right)^{2}\left(1+\rho_{0}^{-2}\right)\right]\left[1+\gamma_{L}^{2}\left(\mathbf{b}_{0} \times \mathbf{V}\right)^{2}\left(1+\rho_{0}^{2}\right)\right]}},
\end{aligned}
$$

where we used the relativistic invariance of $\mathbf{E} \cdot \mathbf{B}$.

It is useful to introduce dimensionless parameters $\gamma, \epsilon$ and $\rho$ as [62]

$$
\gamma=\frac{1}{\rho} \sqrt{\frac{2 \varepsilon_{b}}{m}}, \quad \rho=\frac{E}{B}, \quad \epsilon=\frac{e E}{m^{2}}\left(\frac{m}{2 \varepsilon_{b}}\right)^{3 / 2},
$$

where $m$ is quark mass and $\varepsilon_{b}$ is quarkonium binding energy. I will treat the quarkonium binding potential in the non-relativistic approximation, which provides a very good accuracy to the dissociation rate $[57,62]$. The quarkonium dissociation rate in the comoving frame in the non-relativistic approximation is given by [61]

$$
w=\frac{8 \varepsilon_{b}}{\epsilon} P(\gamma, \theta) C^{2}(\gamma, \theta) e^{-(2 / 3 \epsilon) g(\gamma, \theta)}
$$

where function $g$ reads

$$
g=\frac{3 \tau_{0}}{2 \gamma}\left[1-\frac{1}{\gamma}\left(\frac{\tau_{0}^{2}}{\gamma^{2}}-1\right)^{1 / 2} \sin \theta-\frac{\tau_{0}^{2}}{3 \gamma^{2}} \cos ^{2} \theta\right] .
$$

and functions $P$ and $C$ are given in the following formulas:

$$
\begin{gathered}
P=\frac{\gamma^{2}}{\tau_{0}}\left[\left(\tau_{0} \operatorname{coth} \tau_{0}+\frac{\sinh \tau_{0} \cosh \tau_{0}}{\tau_{0}}-2\right) \sin ^{2} \theta\right. \\
\left.+\sinh ^{2} \tau_{0} \cos ^{2} \theta\right]^{-1 / 2}, \\
C=\exp \left[\ln \frac{\tau_{0}}{2 \gamma}+\int_{0}^{\tau_{0}} d \tau\left(\frac{\gamma}{\xi(\tau)}-\frac{1}{\tau_{0}-\tau}\right)\right], \\
\xi(\tau)=\left\{\frac{1}{4}\left(\tau_{0}^{2}-\tau^{2}\right)^{2} \cos ^{2} \theta\right. \\
+\tau_{0}^{2}\left[\left(\frac{\cosh \tau_{0}-\cos \tau}{\sinh \tau_{0}}\right)^{2}\right. \\
\left.\left.-\left(\frac{\sinh \tau}{\sinh \tau_{0}}-\frac{\tau}{\tau_{0}}\right)^{2}\right] \sin ^{2} \theta\right\}^{1 / 2} .
\end{gathered}
$$


The contribution of quark spin is taken into account by replacing $\varepsilon_{b} \rightarrow \varepsilon_{b}^{\prime}=\varepsilon_{b}-(e / m) \mathbf{s} \cdot \mathbf{B}$ [57]. Function $g$ represents the leading quasi-classical exponent, $P$ is the prefactor for the $S$-wave state of quarkonium, and $C$ accounts for the Coulomb interaction between the valence quarks. Parameter $\tau_{0}$ satisfies the following equation:

$$
\tau_{0}^{2}-\sin ^{2} \theta\left(\tau_{0} \operatorname{coth} \tau_{0}-1\right)^{2}=\gamma^{2}
$$

which establishes its dependence on $\theta$ and $\gamma$. Note that in the limit $E \rightarrow 0$ the dissociation rate (160) exponentially vanishes. This is because pure magnetic field cannot force a charge to tunnel through a potential barrier.

In the case that mechanism (i) is responsible for generation of electric field, $\mathbf{E}_{0}$ is the field permitting the entire plasma in a single event. Event average is then obtained by averaging (160) over an ensemble of events. In the case that mechanism (ii) is operative, averaging is more complicated. Equation (160) gives the quarkonium dissociation rate in a bubble with a given value of $\Theta$. Its derivation assumes that the dissociation process happens entirely inside a bubble and that $\Theta$ is constant inside the bubble. Since in a relativistic heavy-ion collision many bubbles can be produced with a certain distribution of $\Theta$ s (with average $\langle\Theta\rangle=0$ ) more than one bubble can affect the dissociation process. This will result in a distractive interference leading to reduction of the $C P$ odd effect on quarkonium dissociation. However, if a typical bubble size $R_{0}$ is much larger than the size of quarkonium $R_{J}$, then the dissociation is affected by one bubble at a time independently of others, and hence the interference effect can be neglected. In this case (74) provides, upon a proper average, a reasonable estimate of quarkonium dissociation in a heavyion collision. We can estimate the bubble size as the size of the sphaleron, which is of the order of the chromomagnetic screening length $\sim 1 / g^{2} T$, whereas the quarkonium size is of the order $\alpha_{s} / \varepsilon_{b}$. Consequently, at small coupling and below the zero-field dissociation temperature (i.e., when $\varepsilon_{b}$ is not too small) $R_{0}$ is parametrically much larger than $R_{J}$. A more quantitative estimate of the sphaleron size is $R_{0} \simeq 1.2 / \alpha_{s} N_{c} T \simeq 0.4 \mathrm{fm}[79]$; whereas $R_{J} \simeq \alpha_{s} / \varepsilon_{b} \simeq$ $0.1-0.2 \mathrm{fm}$. Thus, based on this estimate bubble interference can be neglected in the first approximation. However, since the ratio $R_{J} / R_{0}$ is actually not so small this effect nevertheless warrants further investigation.

To obtain the experimentally observed $J / \psi$ dissociation rate we need to average (74) over the bubbles produced in a given event and then over all events. To this end it is important to note that because the dissociation rate depends only on $\rho_{0}^{2}$ it is insensitive to the sign of the $\mathbf{E}_{0}$ field, or, in other words, it depends only on absolute value of $\Theta$ but not on its sign. Therefore, it stands to reason that although the precise distribution of $\Theta s$ is not known, (74) gives an approximate event average with parameter $\Theta$ representing a characteristic absolute value of the theta angle.

5.5.3. Limiting Cases. Before I proceed with the numerical calculations, let us consider for illustration several limiting cases. If quarkonium moves with non-relativistic velocity, then in the comoving frame electric and magnetic fields are approximately parallel $\theta \approx 0$, whereas in the ultrarelativistic case they are orthogonal $\theta \approx \pi / 2$; see (158). In the later case the electromagnetic field in the comoving frame does not depend on $E_{0}$ as seen in (157a) and (157b) and therefore the dissociation rate becomes insensitive to the CME. In our estimates I will assume that $\rho_{0}<1$ which is the relevant phenomenological situation. Indeed, it was proposed in [68] that $\rho_{0} \sim \alpha \ll 1$ produces charge fluctuations with respect to the reaction plane of the magnitude consistent with experimental data.

(1) $\theta \gtrsim 0$; that is, electric and magnetic fields are approximately parallel. This situation is realized in the following two cases. (i) Non-relativistic quarkonium velocities: $V \ll \rho_{0}$ or (ii) motion of quarkonium at small angle $\phi$ to the direction of the magnetic field $\mathbf{b}_{0}: \phi \ll \rho_{0} / \gamma_{L} V$. In both cases $E \approx E_{0}$ and $B \approx B_{0}$. This is precisely the case where the dissociation rate exhibits its strongest sensitivity to the strength of the electric field $\mathbf{E}_{0}$ generated by the local parity violating QCD effects. Depending on the value of the $\gamma$ parameter defined in (159) we can distinguish the case of strong electric field $\gamma \gg 1$ and weak electric field $\gamma \ll 1$ [63]. In the former case, $g=(3 / 8) \gamma$, $P=(8 / e)^{1 / 2} \gamma e^{-\gamma^{2} / 2}$, and $C=e^{\pi \gamma / 2} / \gamma$. Substituting into (74) the dissociation rate reads

$$
\begin{aligned}
w & =\frac{8 \varepsilon_{b}}{\epsilon \gamma} \sqrt{\frac{8}{e}} e^{-\gamma^{2} / 2} e^{-\gamma / 4 \epsilon} \\
& =\frac{16 \varepsilon_{b}^{2} m}{e B_{0}} \sqrt{\frac{8}{e}} e^{-\varepsilon_{b} / \rho_{0}^{2} m} e^{-\varepsilon_{b}^{2} / \rho_{0} e E_{0}}, \quad \gamma \gg 1 .
\end{aligned}
$$

In the later case, $g=P=C=1$ and

$$
\begin{aligned}
w & =\frac{8 \varepsilon_{b}}{\epsilon} e^{-2 / 3 \epsilon} \\
& =\frac{8 \varepsilon_{b} m^{2}}{e E_{0}}\left(\frac{2 \varepsilon_{b}}{m}\right)^{3 / 2} e^{-\left(2 m^{2} / 3 e E_{0}\right)\left(2 \varepsilon_{b} / m\right)^{3 / 2}}, \quad \gamma \ll 1,
\end{aligned}
$$

where the electromagnetic field in the comoving frame equals one in the laboratory frame as was mentioned previously.

(2) $\theta \sim \pi / 2$; that is, electric and magnetic fields are approximately orthogonal (note that the limit $\gamma \gg 1$ is different in $\theta=\pi / 2$ and $\theta<(\pi / 2)$ cases [61]). This occurs for an ultra-relativistic motion of quarkonium $V \rightarrow 1$. In this case

$$
B=E=B_{0} \gamma_{L}\left|\mathbf{b}_{0} \times \mathbf{V}\right| \sqrt{1+\rho_{0}^{2}}
$$

This case was discussed in detail in our previous paper [57]. In particular for $\gamma \ll 1$, we get

$$
w=\frac{8 \varepsilon_{b} m^{2}}{e E}\left(\frac{2 \varepsilon_{b}}{m}\right)^{3 / 2} e^{-\left(2 m^{2} / 3 e E\right)\left(2 \varepsilon_{b} / m\right)^{3 / 2}} .
$$

Due to (164) and (167) dependence of $w$ on $E_{0}$ is weak unless $\rho_{0} \gg 1$.

5.6. Dissociation Rate of $J / \psi$. One of the most interesting applications of this formalism is calculation of the dissociation rate of $J / \psi$ which is considered a litmus test of the 
quark-gluon plasma [80]. Let $z$ be the heavy ions collision axis; heavy-ion collision geometry implies that $\mathbf{b}_{0} \cdot \widehat{\mathbf{z}}=0$. The plane containing $z$-axis and perpendicular to the magnetic field direction is the reaction plane. We have

$$
\left(\mathbf{b}_{0} \times \mathbf{V}\right)^{2}=V_{z}^{2}+V_{\perp}^{2} \sin ^{2} \phi
$$

where $\phi$ is the angle between the directions of $\mathbf{B}_{0}$ and $\mathbf{V}_{\perp}$, and I denoted vector components in the $x y$-plane by the subscript $\perp$. We can express the components of the quarkonium velocity $\mathbf{V}$ in terms of the rapidity $\eta$ as $V_{z}=$ $\tanh \eta, V_{\perp}=P_{\perp} /\left(M_{\perp} \cosh \eta\right)$, where $\mathbf{P}$ and $M$ are the quarkonium momentum and mass and $M_{\perp}^{2}=M^{2}+P_{\perp}^{2}$.

Results of numerical calculations are exhibited in Figures 13-15 [58]. In Figure 13 I show the dissociation rate of $J / \psi$ for several values of the electric field $\mathbf{E}_{0}$ induced by the Chiral Magnetic Effect. Note that the typical size of the medium traversed by a quarkonium in magnetic field can be estimated very conservatively as a few fm. Therefore, $w \sim 0.3-0.5 \mathrm{fm}^{-1}$ corresponds to complete destruction of $J / \psi$ s. This means that in the magnetic field of strength $e B_{0} \sim 15 m_{\pi}^{2}$ all $J / \psi$ s with $P_{\perp} \gtrsim 0.5 \mathrm{GeV}$ are destroyed independently of the strength of $E_{0}$. Since magnetic field strength decreases towards the QGP periphery, most of $J / \psi$ surviving at later times originate from that region. Effect of electric field $\mathbf{E}_{0}$ is strongest at low $P_{\perp}$, which is consistent with our discussion in the previous section. The dissociation rate at low $P_{\perp}$ exponentially decreases with decrease of $E_{0}$. Probability of quarkonium ionization by the fields below $E_{0} \lesssim$ $0.1 B_{0}$ (i.e., $\rho_{0} \lesssim 0.1$ ) is exponentially small. This is an order of magnitude higher than the estimate $\rho_{0} \sim \alpha$ of electric field due to CME effect as proposed in [68].

As the plasma temperature varies, so is the binding energy of quarkonium, although the precise form of the function $\varepsilon_{b}(T)$ is model dependent. The dissociation rate picks at some $\varepsilon_{b}^{0}<\varepsilon_{b}^{\text {vac }}$ (see Figure 13(b)), where $\varepsilon_{b}^{\text {vac }}$ is the binding energy in vacuum, indicating that $J / \psi$ breaks down even before $\varepsilon_{b}$ drops to zero, which is the case at $\mathbf{B}_{0}=0$. This $\varepsilon_{b}^{0}$ is a strong function of $E_{0}$ as can be seen in Figure 14. It satisfies the equation $\partial w / \partial \varepsilon_{b}=0$. In the case $\gamma \ll 1$ (165) and (167) imply that

$$
\varepsilon_{b}^{0}=\frac{m}{2}\left(\frac{5 e E}{2 m^{2}}\right)^{2 / 3}, \quad \gamma \ll 1 .
$$

At $\gamma \gg 1$ and $\theta=\pi / 2$ we employ (164) to derive the condition $\left(\varepsilon_{b}^{0}\right)^{2}+e B \varepsilon_{b}^{0} / 2 m-e E^{2} / B=0$. In view of (166) $E \approx B$, and we obtain

$$
\varepsilon_{b}^{0}=\frac{e B}{4 m}\left(\sqrt{\frac{16 m^{2}}{e B}+1}-1\right) \approx \sqrt{e B}, \quad \gamma \gg 1,
$$

where in the last step I used that $e B \ll m^{2}$. For a given function $\varepsilon_{b}(T)$ one can convert $\varepsilon_{b}^{0}$ into the dissociation temperature, which is an important phenomenological parameter.

In the absence of electric fied $\mathbf{E}_{0}$, the dissociation probability peaks in the direction perpendicular to the direction of magnetic field $\mathbf{b}_{0}$, that is, in the reaction plane. Dissociation

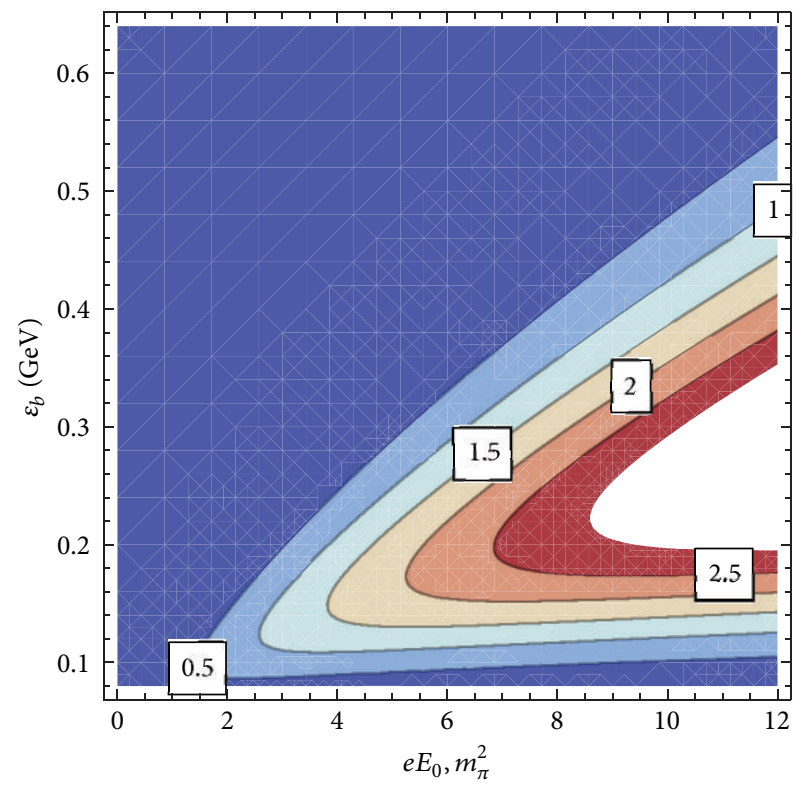

Figure 14: Contour plot of the dissociation rate of $J / \psi$ as a function of $\varepsilon_{b}$ and $e E_{0}$ at $e B_{0}=15 m_{\pi}^{2}, \phi=\pi / 2$ (in the reaction plane), $\eta=0$ (midrapidity), and $P_{\perp}=0.1 \mathrm{GeV}$. Numbers inside boxes indicate the values of $w$ in $1 / \mathrm{fm}$.

rate vanishes in the $\mathbf{b}_{0}$ direction. Indeed, for $\mathbf{V} \cdot \mathbf{b}_{0}=0$ (157a) and (157b) imply that $E=0$. This feature is seen in Figure 15(a). At finite $\mathbf{E}_{0}$ the dissociation probability is finite in the $\mathbf{b}_{0}$ direction making the azimuthal distribution more symmetric. The shape of the azimuthal distribution strongly depends on quarkonium velocity, while at low $V$ the strongest dissociation is in the direction of the reaction plane, at higher $V$ the maximum shifts towards small angles around the $\mathbf{b}_{0}$ direction. Extrema of the azimuthal distribution are roots of the equation $\partial w / \partial \phi=0$. At $\gamma \ll 1$ it yields minimum at $\phi_{0}=0$, maximum at $\phi_{0}=\pi / 2$ and another maximum that satisfies the condition (neglecting the spin-dependence of $\varepsilon_{b}$ )

$$
e E_{0} \sqrt{1+\gamma_{L}^{2}\left(V_{z}^{2}+V_{\perp}^{2} \sin ^{2} \phi_{0}\right)\left(1+\rho_{0}^{-2}\right)}=\frac{2 m^{2}}{3}\left(\frac{2 \varepsilon_{b}}{m}\right)^{3 / 2} .
$$

In order to satisfy (171) $\phi_{0}$ must decrease when $V$ increases and vise versa. This features are seen in Figure 15(a).

Spectrum of quarkonia surviving in the electromagnetic field is proportional to the survival probability $P=1-$ $w t$, where $t$ is the time spent by the quarkonium in the field. Consider $P$ as a function of the angle $\chi$ between the quarkonium velocity and the reaction plane $\chi=\pi / 2-\phi$. Fourier expansion of $P$ in $\chi$ reads

$$
\begin{aligned}
& P(\chi)=\frac{1}{2} P_{0}+\sum_{n=1}^{\infty} P_{n} \cos (n \chi), \\
& P_{n}=\frac{1}{\pi} \int_{-\pi}^{\pi} P(\chi) \cos (n \chi) d \chi .
\end{aligned}
$$




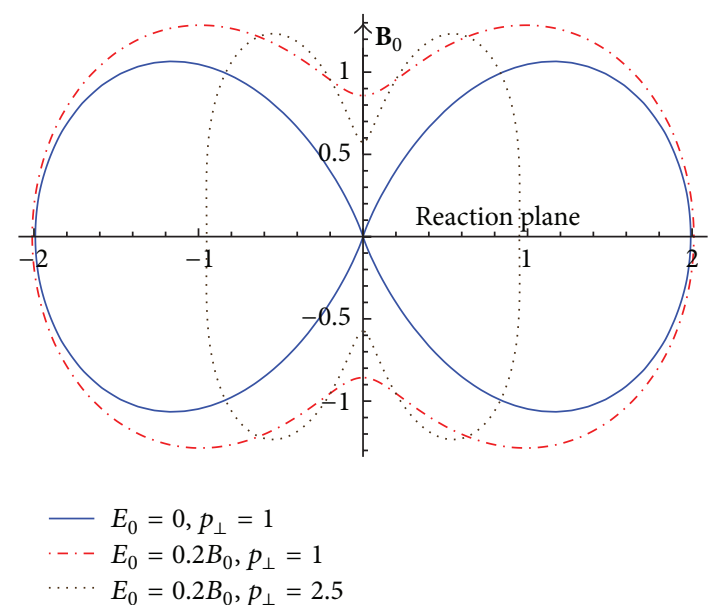

(a)

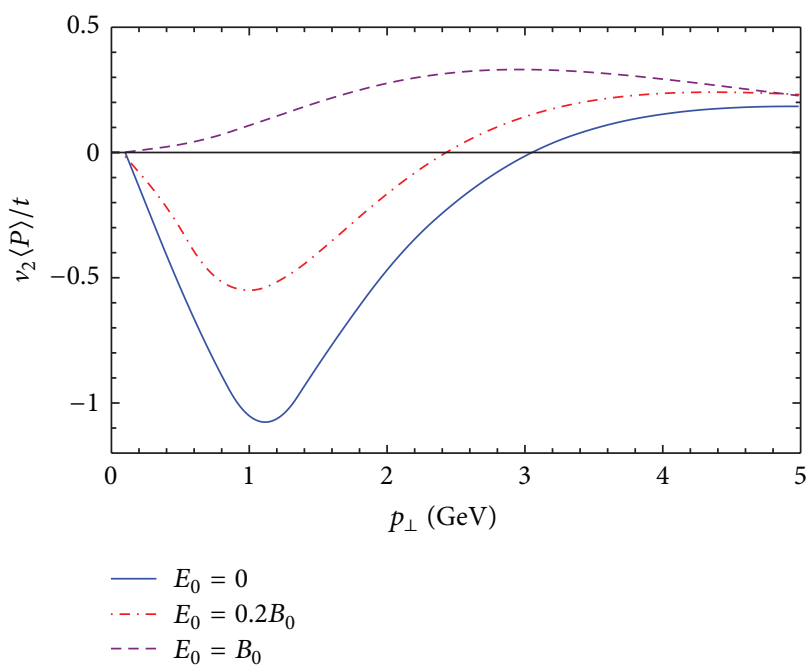

(b)

Figure 15: (a) Angular distribution of $J / \psi$ dissociation rate at $e B_{0}=15 m_{\pi}^{2}, \eta=0$ at different $E_{0}$ and $P_{\perp}$ (in GeV's). Magnetic field $\mathbf{B}_{0}$ points in the positive vertical direction. Reaction plane coincides with the horizontal plane. (b) Rescaled second Fourier-harmonic $v_{2}$ of the azimuthal distribution as a function of $P_{\perp} .\langle P\rangle$ is the azimuthal average of the survival probability, and $t$ is the time spent by $J / \psi$ in the $P$-odd bubble.

Ellipticity of the distribution is characterized by the "elliptic flow" coefficient $v_{2}$ defined as

$$
\begin{aligned}
v_{2} & =\frac{P_{2}}{(1 / 2) P_{0}}=\frac{\int_{-\pi}^{\pi}(1-w t) \cos 2 \chi d \chi}{\pi\langle P\rangle} \\
& =-\frac{t}{\pi\langle P\rangle} \int_{-\pi}^{\pi} w \cos 2 \chi d \chi,
\end{aligned}
$$

where $\langle P\rangle$ denotes average of $P$ over the azimuthal angle. These formulas are applicable only as long as $w t<1$ because otherwise there are no surviving quarkonia. In Figure 15(b) [58] I show $v_{2}\langle P\rangle / t$, which is independent of $t$, as a function of $P_{\perp}$. As expected, in the absence of the CME, $v_{2}$ is negative at low $P_{\perp}$ and positive at high $P_{\perp} \cdot v_{2}$ changes sign at $P_{\perp}$ that depends on the strength of the electric field. It decreases as $E_{0}$ increases until at $E_{0} \simeq B_{0}$ it becomes positive at all $P_{\perp}$. Figure 15(b) provides the low bound for $v_{2}$ because $\langle P\rangle<1$ and $t \geq 1 \mathrm{fm}$. We thus expect that magnetic field strongly modifies the azimuthal distribution of the produced $J / \psi$ s. Role of the magnetic field in generation of azimuthal anisotropies in heavy-ion collisions has been pointed out before in $[29,55]$.

In summary, we observed that $J / \psi$ dissociation energy increases with magnetic field strength and quarkonium momentum. As a consequence, quarkonia dissociate at lower temperature than one would have expected based on calculations neglecting magnetic field $[57,58]$. Figure 13 indicates that in heavy-ion collisions at the LHC, all $J / \psi$ 's moving with $P_{\perp}>0.5 \mathrm{GeV}$ in the reaction plane would dissociate with probability of order unity even if the QGP effect was completely negligible. If electric field fluctuations shown in Figure 3 are taken into account, then even low $P_{\perp} J / \psi$ 's are destroyed. However, chiral magnetic effect has negligible effect on $J / \psi$ dissociation.
Although magnetic fields in $p p$ and $p A$ collisions are much weaker than in $A A$ collisions, they are still strong enough to cause $J / \psi$ dissociation at sufficiently high momenta $P_{\perp}$. A truly spectacular feature of such process would be $J / \psi$ decay into two heavier $D$-mesons.

The effect of $J / \psi$ dissociation in a magnetic field vanishes in the direction parallel to the magnetic field, that is, perpendicular to the reaction plane. Therefore, $J / \psi$ dissociation gives negative contribution to the total azimuthal asymmetry coefficient $v_{2}$. It remarkable that presence of electric field reverses this effect making $v_{2}$ positive.

\section{Electromagnetic Radiation by Quark-Gluon Plasma in Magnetic Field}

6.1. Necessity to Quantize Fermion Motion. In Section 3 we discussed synchrotron radiation of gluons by fast quarks. Our main interest was the energy loss problem. In this section we turn to the problem of electromagnetic radiation by QGP, namely, radiation of photons by thermal fermions [7]. In this case quasi-classical approximation that we employed in Sections 3 and 4 is no longer applicable, and one has to take into account quantization of fermion motion in magnetic field.

Electromagnetic radiation by quarks and antiquarks of QGP moving in external magnetic field originates from two sources: (i) synchrotron radiation and (ii) quark and antiquark annihilation. QGP is transparent to the emitted electromagnetic radiation because its absorption coefficient is suppressed by $\alpha^{2}$. Thus, QGP is shinning in magnetic field. The main goal of this paper is to calculate the spectrum and angular distribution of this radiation. In strong magnetic field it is essential to account for quantization of fermion spectra. Indeed, spacing between the Landau levels is of the order 
$e B / \varepsilon$ ( $\varepsilon$ being quark energy), while their thermal width is of the order $T$. Spectrum quantization is negligible only if $e B / \varepsilon \ll T$ which is barely the case at RHIC and certainly not the case at LHC (at least during the first few fms of the evolution). Fermion spectrum quantization is important not only for hard and electromagnetic probes but also for the bulk properties of QGP.

6.2. Synchrotron Radiation. Motion of charged fermions in external magnetic field, which I will approximately treat as spatially homogeneous, is quasi-classical in the field direction and quantized in the reaction plane, which is perpendicular to the magnetic field and span by the impact parameter and the heavy-ion collision axis. In high energy physics one usually distinguishes the transverse plane, which is perpendicular to the collision axis and span by the magnetic field and the impact parameter. In this section I use notation in which three vectors are discriminated by the bold face and their component along the field direction by the plain face. Momentum projections onto the transverse plane are denoted by subscript $\perp$.

In the configuration space, charged fermions move along spiral trajectories with the symmetry axis aligned with the field direction. Synchrotron radiation is a process of photon $\gamma$ radiation by a fermion $f$ with electric charge $e_{f}=z_{f} e$ in external magnetic field $B$ as follows:

$$
f\left(e_{f}, j, p\right) \longrightarrow f\left(e_{f}, k, q\right)+\gamma(\mathbf{k}),
$$

where $\mathbf{k}$ is the photon momentum, $p, q$ are the momentum components along the magnetic field direction, and indicies $j, k=0,1,2, \ldots$ label the discrete Landau levels in the reaction plane. The Landau levels are given by

$$
\begin{aligned}
& \varepsilon_{j}=\sqrt{m^{2}+p^{2}+2 j e_{f} B}, \\
& \varepsilon_{k}=\sqrt{m^{2}+q^{2}+2 k e_{f} B} .
\end{aligned}
$$

In the constant magnetic field only momentum component along the field direction is conserved. Thus, the conservation laws for synchrotron radiation read

$$
\varepsilon_{j}=\omega+\varepsilon_{k}, \quad p=q+\omega \cos \theta,
$$

where $\omega$ is the photon energy and $\theta$ is the photon emission angle with respect to the magnetic field. Intensity of the synchrotron radiation was derived in [81]. In [82-85] it was thoroughly investigated as a possible mechanism for $\gamma$-ray bursts. In particular, synchrotron radiation in electromagnetic plasmas was calculated. Spectral intensity of angular distribution of synchrotron radiation by a fermion in the $j$ th Landau state is given by

$$
\frac{d I^{j}}{d \omega d \Omega}=\sum_{f} \frac{z_{f}^{2} \alpha}{\pi} \omega^{2} \sum_{k=0}^{j} \Gamma_{j k}\left\{\left|\mathscr{M}_{\perp}\right|^{2}+\left|\mathscr{M}_{\|}\right|^{2}\right\} \delta\left(\omega-\varepsilon_{j}+\varepsilon_{k}\right),
$$

where $\Gamma_{j k}=\left(1+\delta_{j 0}\right)\left(1+\delta_{k 0}\right)$ accounts for the double degeneration of all Landau levels except the ground one.
The squares of matrix elements $\mathscr{M}$, which appear in (177), corresponding to photon polarization perpendicular and parallel to the magnetic field are given by, respectively,

$$
\begin{aligned}
& 4 \varepsilon_{j} \varepsilon_{k}\left|\mathscr{M}_{\perp}\right|^{2} \\
& =\left(\varepsilon_{j} \varepsilon_{k}-p q-m^{2}\right)\left[I_{j, k-1}^{2}+I_{j-1, k}^{2}\right] \\
& +2 \sqrt{2 j e_{f} B} \sqrt{2 k e_{f} B}\left[I_{j, k-1} I_{j-1, k}\right], \\
& 4 \varepsilon_{j} \varepsilon_{k}\left|\mathscr{M}_{\|}\right|^{2} \\
& =\cos ^{2} \theta\left\{\left(\varepsilon_{j} \varepsilon_{k}-p q-m^{2}\right)\left[I_{j, k-1}^{2}+I_{j-1, k}^{2}\right]\right. \\
& \left.-2 \sqrt{2 j e_{f} B} \sqrt{2 k e_{f} B}\left[I_{j, k-1} I_{j-1, k}\right]\right\} \\
& -2 \cos \theta \sin \theta\left\{p \sqrt{2 k e_{f} B}\left[I_{j-1, k} I_{j-1, k-1}+I_{j, k-1} I_{j, k}\right]\right. \\
& \left.+q \sqrt{2 j e_{f} B}\left[I_{j, k} I_{j-1, k}+I_{j-1, k-1} I_{j, k-1}\right]\right\} \\
& +\sin ^{2} \theta\left\{\left(\varepsilon_{j} \varepsilon_{k}+p q-m^{2}\right)\left[I_{j-1, k-1}^{2}+I_{j, k}^{2}\right]\right. \\
& \left.+2 \sqrt{2 j e_{f} B} \sqrt{2 k e_{f} B}\left(I_{j-1, k-1} I_{j, k}\right)\right\},
\end{aligned}
$$

where for $j \geq k$,

$$
I_{j, k} \equiv I_{j, k}(x)=(-1)^{j-k} \sqrt{\frac{k !}{j !}} e^{-x / 2} x^{(j-k) / 2} L_{k}^{j-k}(x),
$$

and $I_{j, k}(x)=I_{k, j}(x)$ when $k>j \cdot\left(I_{j,-1}\right.$ are identically zero.) The functions $L_{k}^{j-k}(x)$ are the generalized Laguerre polynomials. Their argument is

$$
x=\frac{\omega^{2}}{2 e_{f} B} \sin ^{2} \theta .
$$

Angular distribution of radiation is obtained by integrating over the photon energies and remembering that $\varepsilon_{k}$ also depends on $\omega$ by virtue of (175) and (176) as follows:

$$
\begin{aligned}
\frac{d I^{j}}{d \Omega}=\sum_{f} \frac{z_{f}^{2} \alpha}{\pi} \sum_{k=0}^{j} & \frac{\omega^{*}\left(\varepsilon_{j}-\omega^{*}\right)}{\varepsilon_{j}-p \cos \theta-\omega^{*} \sin ^{2} \theta} \\
& \times \Gamma_{j k}\left\{\left|\mathscr{M}_{\perp}\right|^{2}+\left|\mathscr{M}_{\|}\right|^{2}\right\},
\end{aligned}
$$

where photon energy $\omega$ is fixed to be

$$
\begin{aligned}
\omega^{*}=\frac{1}{\sin ^{2} \theta}\{ & \left(\varepsilon_{j}-p \cos \theta\right) \\
& \left.-\left[\left(\varepsilon_{j}-p \cos \theta\right)^{2}-2 e_{f} B(j-k) \sin ^{2} \theta\right]^{1 / 2}\right\} .
\end{aligned}
$$

In the context of heavy-ion collisions the relevant observable is the differential photon spectrum. For ideal plasma in 
equilibrium each quark flavor gives the following contribution to the photon spectrum:

$$
\begin{aligned}
\frac{d N^{\text {synch }}}{d t d \Omega d \omega}= & \sum_{f} \int_{-\infty}^{\infty} d p \frac{e_{f} B\left(2 N_{c}\right) V}{2 \pi^{2}} \\
& \times \sum_{j=0}^{\infty} \sum_{k=0}^{j} \frac{d I^{j}}{\omega d \omega d \Omega}\left(2-\delta_{j, 0}\right) f\left(\varepsilon_{j}\right)\left[1-f\left(\varepsilon_{k}\right)\right],
\end{aligned}
$$

where $2 N_{c}$ accounts for quarks and antiquarks each of $N_{c}$ possible colors, and $\left(2-\delta_{j, 0}\right)$ sums over the initial quark spin.
Index $f$ indicates different quark flavors. $V$ stands for the plasma volume. The statistical factor $f(\varepsilon)$ is

$$
f(\varepsilon)=\frac{1}{e^{\varepsilon / T}+1} .
$$

The $\delta$-function appearing in (177) can be re-written using (175) and (176) as

$$
\delta\left(\omega-\varepsilon_{j}+\varepsilon_{k}\right)=\sum_{ \pm} \frac{\delta\left(p-p_{ \pm}^{*}\right)}{\left|\left(p / \varepsilon_{j}\right)-\left(q / \varepsilon_{k}\right)\right|},
$$

where

$$
p_{ \pm}^{*}=\frac{\left\{\cos \theta\left(m_{j}^{2}-m_{k}^{2}+\omega^{2} \sin ^{2} \theta\right) \pm \sqrt{\left[\left(m_{j}+m_{k}\right)^{2}-\omega^{2} \sin ^{2} \theta\right]\left[\left(m_{j}-m_{k}\right)^{2}-\omega^{2} \sin ^{2} \theta\right]}\right\}}{2 \omega \sin ^{2} \theta} .
$$

The following convenient notation was introduced:

$$
m_{j}^{2}=m^{2}+2 j e_{f} B, \quad m_{k}^{2}=m^{2}+2 k e_{f} B .
$$

The physical meaning of (186) is that synchrotron radiation of a photon with energy $\omega$ at angle $\theta$ by a fermion undergoing transition from $j$ th to $k$ th Landau level is possible only if the initial quark momentum along the field direction equals $p_{+}^{*}$.

Another consequence of the conservation laws (176) is that for a given $j$ and $k$ the photon energy cannot exceed a certain maximal value that will be denoted by $\omega_{s, j k}$. Indeed, inspection of (186) reveals that this equation has a real solution only in two cases:

(i) $m_{j}-m_{k} \geq \omega \sin \theta$, or (ii) $m_{j}+m_{k} \leq \omega \sin \theta$.

The first case is relevant to the synchrotron radiation, while the second one is revelant to the one-photon pair annihilation as discussed in the next section. Accordingly, allowed photon energies in the $j \rightarrow k$ transition satisfy

$$
\omega \leq \omega_{s, j k} \equiv \frac{m_{j}-m_{k}}{\sin \theta}=\frac{\sqrt{m^{2}+2 j e_{f} B}-\sqrt{m^{2}+2 k e_{f} B}}{\sin \theta} .
$$

No synchrotron radiation is possible for $\omega>\omega_{s, j k}$. In particular, when $j=k, \omega_{s, j k}=0$, that is, no photon is emitted, which is also evident in (182). The reason is clearly seen in the frame where $p=0$ : since $\varepsilon_{j} \geq \varepsilon_{k}$, constraints (175) and (176) hold only if $\omega=0$.
Substituting of (177) into (183) yields the spectral distribution of the synchrotron radiation rate per unit volume

$$
\begin{aligned}
& \frac{d N^{\text {synch }}}{V d t d \Omega d \omega} \\
& =\sum_{f} \frac{2 N_{c} z_{f}^{2} \alpha}{\pi^{3}} e_{f} B \\
& \quad \times \sum_{j=0}^{\infty} \sum_{k=0}^{j} \omega\left(1+\delta_{k 0}\right) \vartheta\left(\omega_{s, i j}-\omega\right) \\
& \quad \times \int d p \sum_{ \pm} \frac{\delta\left(p-p_{ \pm}^{*}\right)}{\left|\left(p / \varepsilon_{j}\right)-\left(q / \varepsilon_{k}\right)\right|} \\
& \quad \times\left\{\left|\mathscr{M}_{\perp}\right|^{2}+\left|\mathscr{M}_{\|}\right|^{2}\right\} f\left(\varepsilon_{j}\right)\left[1-f\left(\varepsilon_{k}\right)\right],
\end{aligned}
$$

where $\vartheta$ is the step function.

The natural variables to study the synchrotron radiation are the photon energy $\omega$ and its emission angle $\theta$ with respect to the magnetic field. However, in high energy physics particle spectra are traditionally presented in terms of rapidity $y$ (which for photons is equivalent to pseudo-rapidity) and transverse momentum $k_{\perp} . k_{\perp}$ is a projection of threemomentum $\mathbf{k}$ onto the transverse plane. These variables are not convenient to study electromagnetic processes in external magnetic field. In particular, they conceal the azimuthal symmetry with respect to the magnetic field direction. To change variables, let $z$ be the collision axis, and let $\hat{\mathbf{y}}$ be the direction of the magnetic field. In spherical coordinates photon momentum is given by $\mathbf{k}=\omega(\sin \alpha \cos \phi \widehat{\mathbf{x}}+$ $\sin \alpha \sin \phi \widehat{\mathbf{y}}+\cos \alpha \widehat{\mathbf{z}})$, where $\alpha$ and $\phi$ are the polar and azimuthal angles with respect to $z$-axis. The plane $x z$ is the 


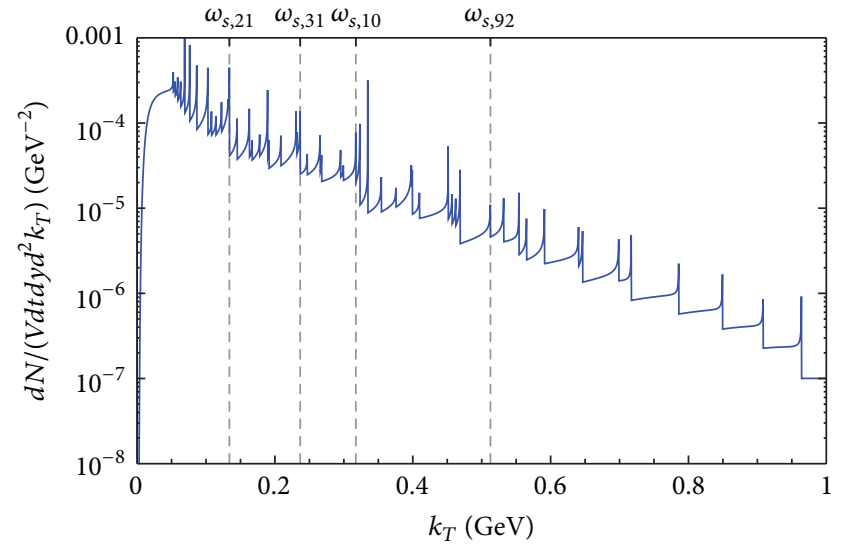

(a)

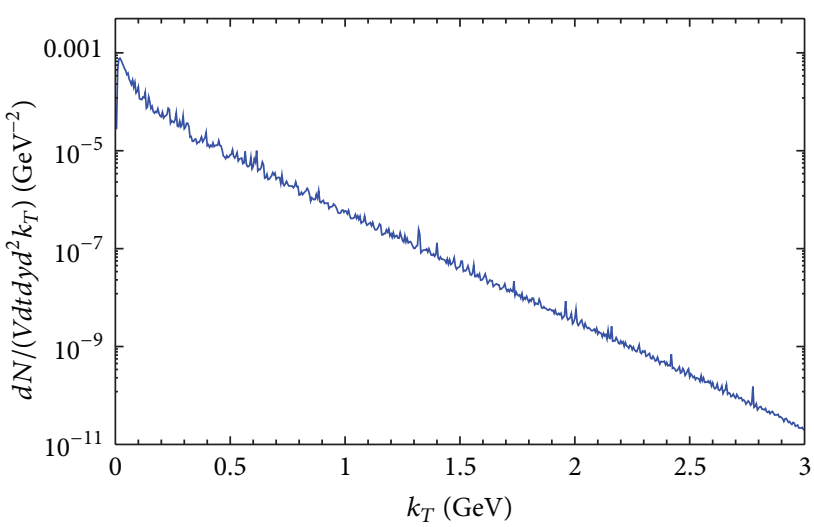

(b)

FIGURE 16: Spectrum of synchrotron radiation by $u$ quarks at $e B=m_{\pi}^{2}, y=0$, and $\phi=\pi / 3$ : (a) contribution of 10 lowest Landau levels $j \leq 10$; several cutoff frequencies are indicated; (b) summed over all Landau levels. $m_{u}=3 \mathrm{MeV}, T=200 \mathrm{MeV}$. Adopted from [7].

reaction plane. By definition, $\widehat{\mathbf{k}} \cdot \widehat{\mathbf{y}}=\cos \theta$ implying that $\cos \theta=\sin \alpha \sin \phi$. Thus,

$$
k_{\perp}=\sqrt{k_{x}^{2}+k_{y}^{2}}=\frac{\omega \cos \theta}{\sin \phi}, \quad y=-\ln \tan \frac{\alpha}{2} .
$$

The second of these equations is the definition of (pseudo-) rapidity. Inverting (191) yields

$$
\omega=k_{\perp} \cosh y, \quad \cos \theta=\frac{\sin \phi}{\cosh y} .
$$

Because $d y=d k_{z} / \omega$ the photon multiplicity in a unit volume per unit time reads

$$
\frac{d N^{\text {synch }}}{d V d t d^{2} k_{\perp} d y}=\omega \frac{d N^{\text {synch }}}{d V d t d^{3} k}=\frac{d N^{\text {synch }}}{d V d t \omega d \omega d \Omega} .
$$

Figure 16 displays the spectrum of synchrotron radiation by $u$ quarks as a function of $k_{\perp}$ at fixed $\phi$ [7]. At midrapidity $y=0$ (192) implies that $k_{\perp}=\omega$. Contribution of $d$ and $s$ quarks is qualitatively similar. At $e B \gg m^{2}$, quark masses do not affect the spectrum much. The main difference stems from the difference in electric charge. In panel (a) only the contributions of the first ten Landau levels are displayed. The cutoff frequencies $\omega_{s, j k}$ can be clearly seen, and some of them are indicated on the plot for convenience. The azimuthal distribution is shown in Figure 17. Note that at midrapidity $\phi=\pi / 2-\theta$. Therefore, the figure indicates that photon production in the direction of magnetic field (at $\phi=\pi / 2$ ) is suppressed. More photons are produced in the direction of the reaction plane $\phi=0$. This results in the ellipticity of the photon spectrum that translates into the positive "elliptic flow" coefficient $v_{2}$. It should be noted that the classical synchrotron radiation has a similar angular distribution.

In order to compare the photon spectrum produced by synchrotron radiation to the photon spectrum measured in heavy-ion collisions, the $u, d$, and $s$ quarks contributions were summed up. Furthermore, the experimental data from [8]

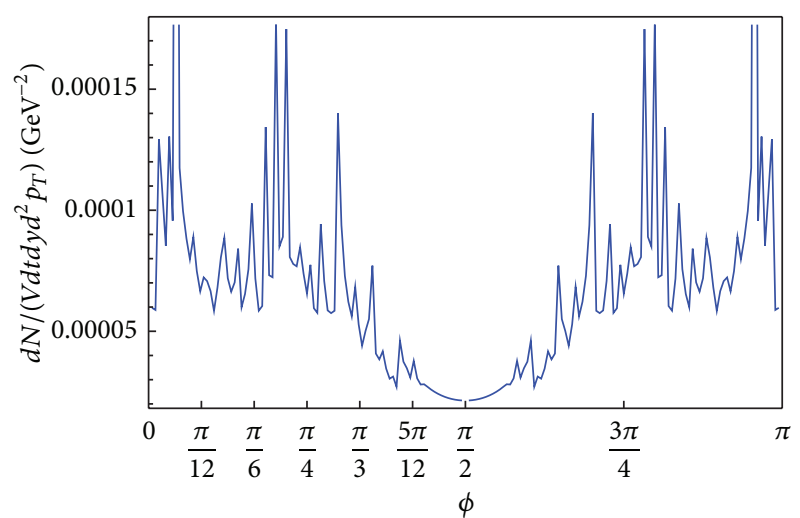

FIGURE 17: Azimuthal distribution of synchrotron radiation by $u$ quarks at $k_{\perp}=0.2 \mathrm{GeV}, e B=m_{\pi}^{2}$, and $y=0 . m_{u}=3 \mathrm{MeV}$. Adopted from [7].

was divided by $V t$, where $t$ is the magnetic field relaxation time. The volume of the plasma can be estimated as $V=\pi R^{2} t$ with $R \approx 5 \mathrm{fm}$ being the nuclear radius. Therefore,

$$
\begin{aligned}
\frac{d N_{\exp }^{\gamma}}{d V d t d^{2} k_{\perp} d y} & =\frac{d N_{\exp }^{\gamma}}{d^{2} k_{\perp} d y} \frac{1}{\pi R^{2} t^{2}} \\
& =\frac{d N_{\exp }^{\gamma}}{d^{2} k_{\perp} d y}\left(\frac{\mathrm{GeV}}{14.9}\right)^{4}\left(\frac{1 \mathrm{fm}}{t}\right)^{2} .
\end{aligned}
$$

The results are plotted in Figure 18. In panel (a) it is seen that synchrotron radiation gives a significant contribution to the photon production in heavy-ion collisions at RHIC energy. This contribution is larger at small transverse momenta. This may explain enhancement of photon production observed in [8]. Panel (b) indicates the increase of the photon spectrum produced by the synchrotron radiation mechanism at the LHC energy. This increase is due to enhancement of the magnetic field strength, but mostly because of increase of plasma temperature. This qualitative features can be better 


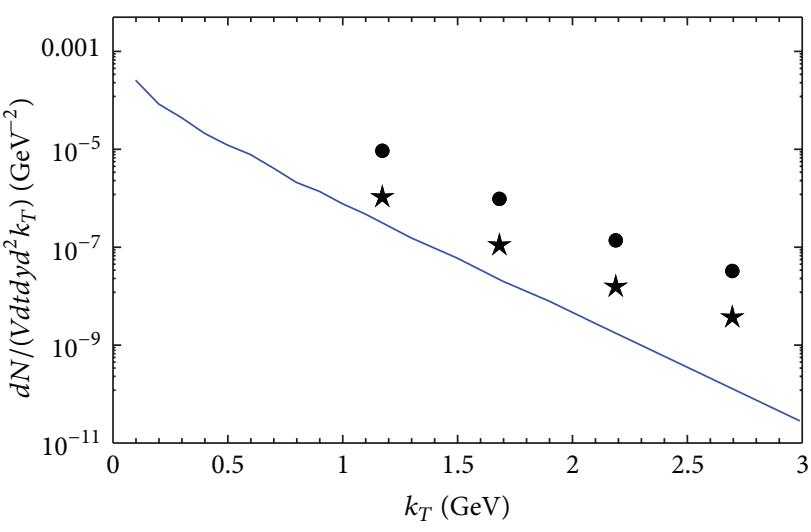

(a)

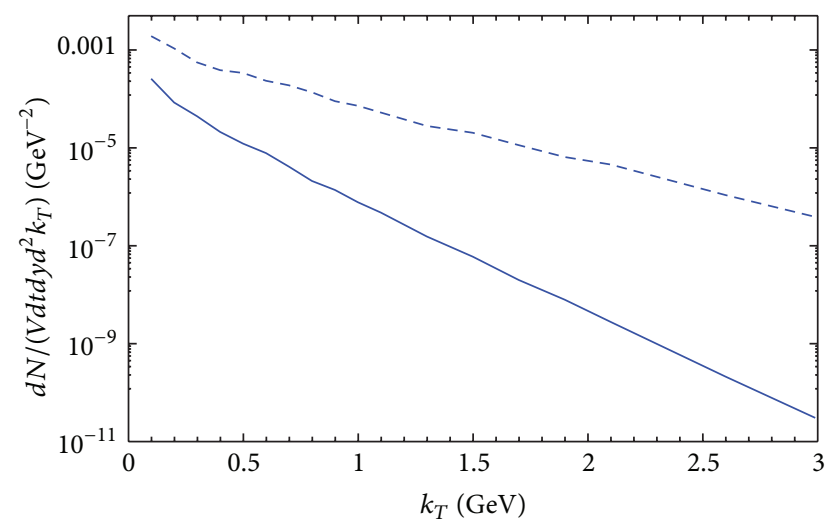

(b)

FIGURE 18: Azimuthal average of the synchrotron radiation spectrum of $u, d, s$ quarks and their corresponding antiquarks. (a) $e B=m_{\pi}^{2}, y=0$ compared to the experimental data from [8] divided by $V t=25 \pi \mathrm{fm}^{4}$ (dots) and $V t=9 \times 25 \pi \mathrm{fm}^{4}$ (stars); (b) $e B=m_{\pi}^{2}, T=200 \mathrm{MeV}$, and $y=0$ (solid line) compared to $e B=15 m_{\pi}^{2}, T=400 \mathrm{MeV}$, and $y=0$ (dashed line). $m_{u}=3 \mathrm{MeV}, m_{d}=5 \mathrm{MeV}$, and $m_{s}=92 \mathrm{MeV}$. Adopted from [7].

understood by considering the limiting cases of low and high photon energies.

One possible way to ascertain the contribution of electromagnetic radiation in external magnetic field is to isolate the azimuthally symmetric component with respect to the direction of the magnetic field. It seems that synchrotron radiation dominates the photon spectrum at low $k_{\perp}$. Thus, azimuthal symmetry can be easily checked by simply plotting the multiplicity versus $\omega, \theta$, and $\varphi$, where $\omega$ is photon energy, $\theta$ is emission angle with respect to the magnetic field, and $\varphi$ is azimuthal angle around the magnetic field direction (which is perpendicular both to the collision axis and to the impact parameter). In Figure 16(a) it is also seen that in these variables it may be possible to discern the cutoff frequencies $\omega_{s, j k}$ that appear as resonances (in Figure $16 y=0$ so $k_{\perp}=\omega$ ). Note that averaging over the azimuthal angle $\alpha$ around the collision axis direction destroys these features; see Figure 18.

6.2.1. Low Photon Energy. The low energy part of the photon spectrum satisfies the condition $\omega \ll \sqrt{e_{f} B}$. The corresponding initial quark momentum components along the field $p$ and energy $\varepsilon_{j}$ follow from (186) and (175) and are given by

$$
\begin{gathered}
p_{ \pm}^{*} \approx \frac{(j-k) e_{f} B(\cos \theta \pm 1)}{\omega \sin ^{2} \theta}+\mathcal{O}(\omega), \\
\varepsilon_{j} \approx\left|p_{ \pm}^{*}\right|+\mathcal{O}(\omega) .
\end{gathered}
$$

Evidently, $\varepsilon_{j} \gg e B$. In practice, magnetic field strength satisfies $\sqrt{e B} \gtrsim T$, so that $\varepsilon_{j} \gg T$. Therefore, synchrotron radiation is dominated by fermion transitions from low Landau levels due to the statistical factors appearing in (183).
For a qualitative discussion it is sufficient to consider the $1 \rightarrow 0$ transition. In this case the matrix elements (178) read

$$
\begin{aligned}
\left|\mathscr{M}^{1,0}\right|^{2}=\frac{1}{2 \varepsilon_{1} \varepsilon_{0}}\{ & I_{1,0}^{2}\left(\varepsilon_{1} \varepsilon_{0}-p q \cos ^{2} \theta-m^{2}\right) \\
& \left.+\cos \theta \sin \theta q \sqrt{2 \mathrm{e}_{f} B} I_{1,0} I_{0,0}\right\} .
\end{aligned}
$$

Assuming that the field strength is supercritical; that is, $e_{f} B \gg m^{2}$, but keeping all powers of $\omega$ (for future reference) (186) reduces to

$$
p_{ \pm}^{*} \approx \frac{1}{2 \omega \sin ^{2} \theta}\left\{2 e_{f} B(\cos \theta \pm 1)+\omega^{2} \sin ^{2} \theta(\cos \theta \mp 1)\right\} .
$$

Furthermore, using the conservation laws (176) we obtain in this approximation

$$
\begin{gathered}
\varepsilon_{1 \pm}=\frac{1}{2 \omega \sin ^{2} \theta}\left|2 e_{f} B(\cos \theta \pm 1)-\omega^{2} \sin ^{2} \theta(\cos \theta \mp 1)\right| \\
q_{ \pm}=\frac{1}{2 \omega \sin ^{2} \theta}\left(2 e_{f} B-\omega^{2} \sin ^{2} \theta\right)(\cos \theta \pm 1), \\
\varepsilon_{0 \pm}=|q| .
\end{gathered}
$$

The values of the nonvanishing matrix elements $I_{j, k}$ defined by (179) are

$$
I_{1,0}(x)=-x^{1 / 2} e^{-x / 2}, \quad I_{0,0}(x)=e^{-x / 2} .
$$


Advances in High Energy Physics

TABLE 1: The upper summation limit in (190) that yields the $5 \%$ accuracy. $j_{\max }$ is the highest Landau level of the initial quark that is taken into account at this accuracy. Throughout the table $y=0$.

\begin{tabular}{|c|c|c|c|c|c|c|c|c|c|c|}
\hline$f$ & $u$ & $u$ & $u$ & $u$ & $u$ & $u$ & $s$ & $u$ & $u$ & $s$ \\
\hline$e B / m_{\pi}^{2}$ & 1 & 1 & 1 & 1 & 1 & 1 & 1 & 15 & 15 & 15 \\
\hline$T, \mathrm{GeV}$ & $\begin{array}{c}0.2 \\
\pi\end{array}$ & $\begin{array}{c}0.2 \\
\pi\end{array}$ & $\begin{array}{c}0.2 \\
\pi\end{array}$ & $\begin{array}{c}0.2 \\
\pi\end{array}$ & $\begin{array}{c}0.2 \\
\pi\end{array}$ & $\begin{array}{c}0.2 \\
\pi\end{array}$ & $\begin{array}{c}0.2 \\
\pi\end{array}$ & $\begin{array}{c}0.4 \\
\pi\end{array}$ & $\begin{array}{c}0.4 \\
\pi\end{array}$ & $\begin{array}{c}0.4 \\
\pi\end{array}$ \\
\hline$\phi$ & $\overline{3}$ & $\overline{3}$ & $\overline{3}$ & $\overline{3}$ & $\overline{6}$ & $\overline{12}$ & $\overline{3}$ & $\overline{3}$ & $\overline{3}$ & $\overline{3}$ \\
\hline$k_{\perp}, \mathrm{GeV}$ & 0.1 & 1 & 2 & 3 & 1 & 1 & 1 & 1 & 2 & 1 \\
\hline$x$ & 0.096 & 9.6 & 38 & 86 & 29 & 35 & 19 & 0.64 & 2.6 & 1.3 \\
\hline$j_{\max }$ & 30 & 40 & 90 & 150 & 120 & 200 & 90 & 8 & 12 & 16 \\
\hline
\end{tabular}

For $j=1, k=0$ we write using (189) $\omega_{s, 10}=\sqrt{2 e_{f} B} / \sin \theta$. Then (180) implies $x=\omega^{2} / \omega_{s, 10}^{2}$. Substituting (197)-(199) into (196) gives

$$
\begin{aligned}
\left|\mathscr{M}_{ \pm}^{1,0}\right|^{2}=\frac{1}{2} x e^{-x}[ & 1-\frac{\cos \theta(1+x) \pm(1-x)}{\cos \theta(1-x) \pm(1+x)} \cos ^{2} \theta \\
& \left.-\frac{2(1-x) \cos \theta \sin ^{2} \theta}{\cos \theta(1-x) \pm(1+x)}\right]
\end{aligned}
$$

According to (190) the contribution of the $1 \rightarrow 0$ transition to the synchrotron radiation reads [7]

$$
\begin{aligned}
& \frac{d N^{\text {synch, } 10}}{V d t d \Omega d \omega} \\
& =\sum_{f} \frac{2 N_{c} z_{f}^{2} \alpha}{\pi} \omega \Gamma \frac{e_{f} B}{2 \pi^{2}} \sum_{ \pm} f\left(\varepsilon_{1}\right)\left[1-f\left(\varepsilon_{0}\right)\right]\left|\mathscr{M}_{ \pm}^{1,0}\right|^{2} \\
& \quad \times \frac{(1-x) \cos \theta \pm(1+x)}{-2 x(\cos \theta \mp 1)} \vartheta\left(\omega_{s, 10}-\omega\right) .
\end{aligned}
$$

Consider radiation spectrum at $\theta=\pi / 2$, that is, perpendicular to the magnetic field. The spectrum increases with $x$ and reaches maximum at $x=1$. Since $x=\omega^{2} /\left(2 e_{f} B\right)$, spectrum decreases with increase of $B$ at fixed $\omega$. This feature holds at low $x$ part of the spectrum for other emission angles and even for transitions form higher excited states. However, at high energies, it is no longer possible to approximate the spectrum by the contribution of a few low Landau levels. In that case the typical values of quantum numbers are $j, k \gg 1$. For example, to achieve the numerical accuracy of $5 \%$, sum over $j$ must run up to a certain $j_{\max }$. Some values of $j_{\max }$ are listed in Table 1 [7].

6.2.2. High Photon Energy. The high energy tail of the photon spectrum is quasi-classical and approximately continuous. In this case the Laguerre polynomials can be approximated by the Airy functions or the corresponding modified Bessel functions. The angular distribution of the spectrum can be found in [82]

$$
\frac{d N^{\text {synch }}}{V d t d \Omega d \omega}=\sum_{f} \frac{z_{f}^{2} \alpha}{\pi} \frac{n_{f} \omega m^{2}}{4 T^{3}} \sqrt{\frac{e_{f} B T \sin \theta}{m^{3}}} e^{-\omega / T},
$$

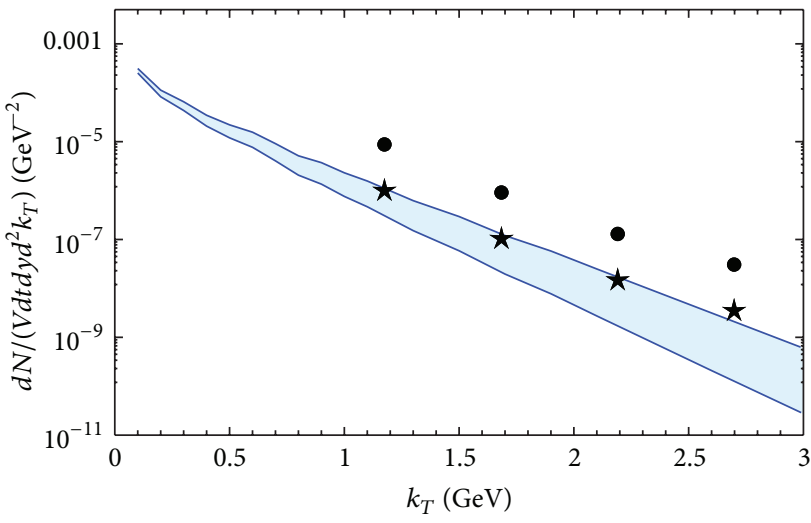

FIGURE 19: Variation of the synchrotron spectrum with plasma temperature. Lower line: $T=200 \mathrm{MeV}$, upper line: $T=250 \mathrm{MeV}$; Other parameters are the same as in Figure 18(a). Adopted from [7].

provided that $\omega \gg m \sqrt{m T / e_{f} B \sin \theta}$. Here $n_{f}$ is number density of flavor $f$, which is independent of $B$ as follows:

$$
n_{f}=\frac{2 \cdot 2 N_{c} e_{f} B}{4 \pi^{2}} \sum_{j=0}^{\infty} \int_{-\infty}^{\infty} d p e^{-\varepsilon_{j} / T} \approx \frac{4 N_{c}}{\pi^{2}} T^{3} .
$$

Here summation over $j$ was replaced by integration. It follows that this part of the spectrum increases with magnetic field strength as $\sqrt{B}$ and with temperature as $\sqrt{T} e^{-\omega / T}$. Therefore, variation of the spectrum with $T$ is much stronger than with $B$. The $T$ dependence is shown in Figure 19.

Unlike time-dependence of magnetic field, timedependence of temperature is non-negligible even during the first few fm/c. Final synchrotron spectrum, which is an average over all temperatures, is dominated by high temperatures/early times. However, the precise form of time-dependence of temperature is model-dependent. Therefore, the spectrum is presented at fixed temperatures, so that a reader can appreciate its qualitative features in a model-independent way.

6.3. Pair Annihilation. The theory of one-photon pair annihilation was developed in $[86,87]$. It was shown in [88] that in the super-critical regime $e B \gg m^{2}$ one-photon annihilation is much larger than the two-photon annihilation. In this section the one-photon annihilation of $q$ and $\bar{q}$ pairs in the QGP is calculated. 


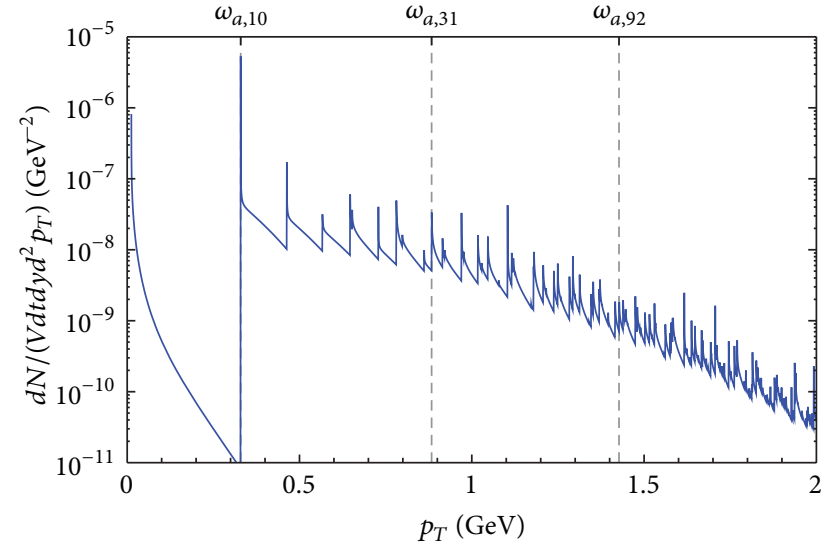

(a)

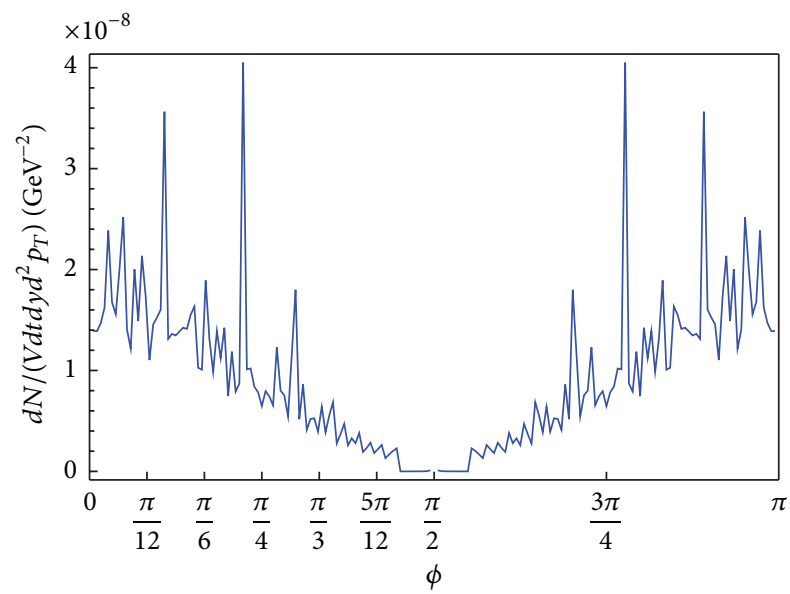

(b)

FIgURE 20: Photon spectrum in one-photon annihilation of $u$ and $\bar{u}$ quarks. $e B=m_{\pi}^{2}, y=0$. (a) $k_{\perp}$-spectrum at $\phi=\pi / 3$, (b) azimuthal angule distribution at $k_{\perp}=1 \mathrm{GeV}$. Adopted from [7].

For $q \bar{q}$ pair annihilation the conservation of energy and momentum is given by

$$
\varepsilon_{j}+\varepsilon_{k}=\omega, \quad p+q=\omega \cos \theta .
$$

The spectral density of the annihilation rate per unit volume reads

$$
\begin{aligned}
& \frac{d N^{\text {annih }}}{V d t d \omega d \Omega} \\
& =\sum_{f} \frac{\alpha z_{f}^{2} \omega N_{c}}{4 \pi e_{f} B} \sum_{j=0}^{\infty} \sum_{k=0}^{\infty} \int d p \frac{2 e_{f} B}{2 \pi^{2}} f\left(\varepsilon_{j}\right) \int d q \frac{2 e_{f} B}{2 \pi^{2}} f\left(\varepsilon_{k}\right) \\
& \quad \times \delta(p+q-\omega \cos \theta) \delta\left(\varepsilon_{j}+\varepsilon_{k}-\omega\right) \\
& \quad \times\left\{\left|\mathscr{T}_{\perp}\right|^{2}+\left|\mathscr{T}_{\|}\right|^{2}\right\},
\end{aligned}
$$

where the matrix elements $\mathscr{T}$ can be obtained from (178) by making substitutions $\varepsilon_{k} \rightarrow-\varepsilon_{k}, q \rightarrow-q$ and are given by

$$
\begin{aligned}
& 4 \varepsilon_{j} \varepsilon_{k}\left|\mathscr{T}_{\perp}\right|^{2} \\
& =\left(\varepsilon_{j} \varepsilon_{k}-p q+m^{2}\right)\left[I_{j, k-1}^{2}+I_{j-1, k}^{2}\right] \\
& -2 \sqrt{2 j e_{f} B} \sqrt{2 k e_{f} B}\left[I_{j, k-1} I_{j-1, k}\right], \\
& 4 \varepsilon_{j} \varepsilon_{k}\left|\mathscr{T}_{\|}\right|^{2} \\
& =\cos ^{2} \theta\left\{\left(\varepsilon_{j} \varepsilon_{k}-p q+m^{2}\right)\left[I_{j, k-1}^{2}+I_{j-1, k}^{2}\right]\right. \\
& \left.\quad+2 \sqrt{2 j e_{f} B} \sqrt{2 k e_{f} B}\left[I_{j, k-1} I_{j-1, k}\right]\right\}
\end{aligned}
$$

$$
\begin{aligned}
&-2 \cos \theta \sin \theta\{-p \sqrt{2 k e_{f} B}\left[I_{j-1, k} I_{j-1, k-1}+I_{j, k-1} I_{j, k}\right] \\
&\left.+q \sqrt{2 j e_{f} B}\left[I_{j, k} I_{j-1, k}+I_{j-1, k-1} I_{j, k-1}\right]\right\} \\
&+\sin ^{2} \theta\left\{\left(\varepsilon_{j} \varepsilon_{k}+p q+m^{2}\right)\left[I_{j-1, k-1}^{2}+I_{j, k}^{2}\right]\right. \\
&\left.-2 \sqrt{2 j e_{f} B} \sqrt{2 k e_{f} B}\left(I_{j-1, k-1} I_{j, k}\right)\right\},
\end{aligned}
$$

with the same functions $I_{i, j}$ as in (179). Integration over $q$ removes the delta function responsible for the conservation of momentum along the field direction. The remaining delta function is responsible for energy conservation and can be written in exactly the same form as in (185) with particle energies and momenta now obeying the conservation laws (204). It is straightforward to see that momentum $p_{ \pm}^{*}$ is still given by (190), (187). The photon spectrum produced by annihilation of quark in state $j$ with antiquark in state $k$ has a threshold $\omega_{a, i j}$ that is given by the case (ii) in (188) as follows:

$$
\omega \geq \omega_{a, i j}=\frac{m_{j}+m_{k}}{\sin \theta}=\frac{\sqrt{m^{2}+2 j e_{f} B}+\sqrt{m^{2}+2 k e_{f} B}}{\sin \theta} .
$$

Thus, the spectral density of the annihilation rate per unit volume is

$$
\begin{aligned}
& \frac{d N^{\text {annih }}}{V d t d \omega d \Omega} \\
& =\sum_{f} \frac{\alpha z_{f}^{2} \omega N_{c}}{4 \pi^{5}} e_{f} B \sum_{j=0}^{\infty} \sum_{k=0}^{\infty} \vartheta\left(\omega-\omega_{a, i j}\right) \\
& \quad \times \int d p \sum_{ \pm} \frac{\delta\left(p-p_{ \pm}^{*}\right)}{\left|\left(p / \varepsilon_{j}\right)-\left(q / \varepsilon_{k}\right)\right|} \\
& \times\left\{\left|\mathscr{T}_{\perp}\right|^{2}+\left|\mathscr{T}_{\|}\right|^{2}\right\} f\left(\varepsilon_{j}\right) f\left(\varepsilon_{k}\right) .
\end{aligned}
$$


Passing to $y$ and $p_{\perp}$ variables in place of $\omega$ and $\theta$ is similar to (193).

The results of the numerical calculations are represented in Figure 20. Panel (a) shows the spectrum of photons radiated in annihilation of $u$ and $\bar{u}$. We conclude that contribution of the annihilation channel is negligible as compared to the synchrotron radiation.

In summary, results of the calculations presented in this section indicate that photon production by QGP due to its interaction with external magnetic field gives a considerable contribution to the total photon multiplicity in heavy-ion collisions. This is seen in Figure 18 were the model calculation is compared with the experimental data [8]. The largest contribution to the photon multiplicity arises from photon momenta of the order of $\sqrt{e B}$. This may provide an explanation of the photon excess observed by the PHENIX experiment [8]. Similar mechanism may also be responsible for enhancement of low mass di-lepton production that proceeds via emission of virtual photon which subsequently decays into dilepton pair.

\section{Summary}

Analytical and numerical calculations indicate existence of extremely powerful electromagnetic fields in relativistic heavy-ion collisions. They are the strongest electromagnetic fields that exist in nature. They evolve slowly on characteristic QGP time scale and therefore have a profound effect on dynamics of QGP. In this review I described the recent progress in understanding of particle production in presence of these fields. Treating the fields as quasi-static and spatially homogeneous allowed us to use analytical results derived over the past half century. This is, however, the main source of uncertainty that can be clarified only in comprehensive numerical approach based on relativistic magnetohydrodynamics.

I discussed many spectacular effects caused by magnetic field. All of them have direct phenomenological relevance. Breaking of spherical symmetry by magnetic field in the direction perpendicular to the collision axis results in azimuthal asymmetry of particle production in the reaction plane. Fast quarks moving in magnetic field radiate a significant fraction of their energy. All electromagnetic probes are also naturally affected by magnetic field. Therefore, all experimental processes that are being used to study the properties of QGP have strong magnetic field dependence. In addition, the QCD phase diagram is modified by magnetic field as has been extensively studied using model calculations [89-110] and lattice simulations [111-123]. Entanglement of effects produced by magnetic field with conventional QGP ones makes it difficult to quantify the role of magnetic field in QGP dynamics. A unique observable is polarization of leptons escaping from QGP, which can be induced only by magnetic field see Section 3.3.

Profound influence of magnetic field on properties of QGP is truly remarkable. Hopefully, progress in theory will soon be matched by experimental investigations that will eventually discover properties of QCD at high temperatures and strong electromagnetic fields.

\section{Acknowledgment}

This work was supported in part by the U.S. Department of Energy under Grant no. DE-FG02-87ER40371.

\section{References}

[1] B. A. Boyko, A. I. Bykov, M. I. Dolotenko et al., "With record magnetic fields to the 21st century," in Proceedings of the 12th IEEE International Pulsed Power Conference, pp. 746-749, June 1999.

[2] C. Kouveliotou, R. C. Duncan, and C. Thompson, "Feature," Scientific American, vol. 288, no. 2, pp. 34-41, 2003.

[3] J. S. Schwinger, "On gauge invariance and vacuum polarization," Physical Review, vol. 82, pp. 664-679, 1951.

[4] A. Bzdak and V. Skokov, "Event-by-event fluctuations of magnetic and electric fields in heavy ion collisions," Physics Letters $B$, vol. 710, pp. 171-174, 2012.

[5] D. E. Kharzeev, L. D. McLerran, and H. J. Warringa, "The effects of topological charge change in heavy ion collisions: 'event by event P and CP violation,' Nuclear Physics A, vol. 803, no. 3-4, pp. 227-253, 2008.

[6] Y. Kluger, J. M. Eisenberg, B. Svetitsky, F. Cooper, and E. Mottola, "Fermion pair production in a strong electric field," Physical Review D, vol. 45, no. 12, pp. 4659-4671, 1992.

[7] K. Tuchin, "Electromagnetic radiation by quark-gluon plasma in a magnetic field," Physical Review C, vol. 87, Article ID 024912, 8 pages, 2013.

[8] A. Adare, S. Afanasiev, C. Aidala et al., "Enhanced production of direct photons in $\mathrm{Au}+\mathrm{Au}$ collisions at $\sqrt{s_{N N}}=200 \mathrm{GeV}$ and implications for the initial temperature," Physical Review Letters, vol. 104, no. 13, Article ID 132301, 6 pages, 2010.

[9] J. Ambjorn and P. Olesen, "W-condensate formation in high energy collisions," Physics Letters B, vol. 257, pp. 201-206, 1991.

[10] P. Olesen, "W condensation atthe LHC?" In press, http://arxiv.org/abs/1207.7045.

[11] V. V. Skokov, A. Y. Illarionov, and V. D. Toneev, "Estimate of the magnetic field strength in heavy-ion collisions," International Journal of Modern Physics A, vol. 24, no. 31, pp. 5925-5932, 2009.

[12] V. Voronyuk, V. D. Toneev, W. Cassing, E. L. Bratkovskaya, V. P. Konchakovski, and S. A. Voloshin, "Electromagnetic field evolution in relativistic heavy-ion collisions," Physical Review $C$, vol. 83, no. 5, Article ID 054911, 2011.

[13] W.-T. Deng and X.-G. Huang, "Event-by-event generation of electromagnetic fields in heavy-ion collisions," Physical Review C, vol. 85, Article ID 044907, 8 pages, 2012.

[14] T. Lappi and L. McLerran, "Some features of the glasma," Nuclear Physics A, vol. 772, no. 3-4, pp. 200-212, 2006.

[15] J.-P. Blaizot, F. Gelis, J. Liao, L. McLerran, and R. Venugopalan, "Thermalization and bose-einstein condensation in overpopulated glasma," In press, http://arxiv.org/abs/1210.6838.

[16] H. T. Ding, A. Francis, O. Kaczmarek, F. Karsch, E. Laermann, and W. Soeldner, "Thermal dilepton rate and electrical conductivity: an analysis of vector current correlation functions in quenched lattice QCD," In press, http://arxiv.org/abs/1012.4963.

[17] G. Aarts, C. Allton, J. Foley, S. Hands, and S. Kim, "Spectral functions at small energies and the electrical conductivity in hot quenched lattice QCD," Physical Review Letters, vol. 99, no. 2, Article ID 022002, 4 pages, 2007.

[18] S. Gupta, "Analyticity and the phase diagram of QCD," Physics Letters B, vol. 588, no. 1-2, pp. 136-144, 2004. 
[19] J. D. Jackson, Classical Electrodynamics, 3rd edition, 1998.

[20] J. D. Bjorken, "Highly relativistic nucleus-nucleus collisions: the central rapidity region," Physical Review D, vol. 27, no. 1, pp. 140151, 1983.

[21] K. Tuchin, "Synchrotron radiation by fast fermions in heavy-ion collisions," Physical Review C, vol. 83, no. 3, Article ID 039903, 1 pages, 2011, Erratum: Physical Review C, vol. 82, article ID 034904, 2010.

[22] G. V. Dunne and C. Schubert, "Worldline instantons and pair production in inhomogenous fields," Physical Review D, vol. 72, no. 10, Article ID 105004, 12 pages, 2005.

[23] R.-C. Wang and C. Y. Wong, "Finite-size effect in the Schwinger particle-production mechanism," Physical Review D, vol. 38, pp. 348-359, 1988.

[24] C. Martin and D. Vautherin, "Finite-size and dynamical effects in pair production by an external field," Physical Review D, vol. 40, pp. 1667-1673, 1989.

[25] S. P. Kim and D. N. Page, "Improved approximations for fermion pair production in inhomogeneous electric fields," Physical Review D, vol. 75, Article ID 045013, 9 pages, 2007.

[26] F. Cooper, J. M. Eisenberg, Y. Kluger, E. Mottola, and B. Svetitsky, "Particle production in the central rapidity region," Physical Review D, vol. 48, no. 1, pp. 190-208, 1993.

[27] Y. Kluger, J. M. Eisenberg, B. Svetitsky, F. Cooper, and E. Mottola, "Pair production in a strong electric field," Physical Review Letters, vol. 67, no. 18, pp. 2427-2430, 1991.

[28] N. Tanji, "Dynamical view of pair creation in uniform electric and magnetic fields," Annals of Physics, vol. 324, no. 8, pp. 16911736, 2009.

[29] R. K. Mohapatra, P. S. Saumia, and A. M. Srivastava, "Enhancement of flow anisotropies due to magnetic field in relativistic heavy-ion collisions," Modern Physics Letters A, vol. 26, p. 2477, 2011.

[30] E. M. Lifshitz and L. P. Pitaevskiy, Physical Kinetics, Pergamon Press, 1981.

[31] H. Van Erkelens and W. A. Van Leeuwen, "Relativistic Boltzmann theory for a plasma: I. The entropy production," Physica A, vol. 89, pp. 113-126, 1977.

[32] H. Van Erkelens and W. A. Van Leeuwen, "Relativistic Boltzmann theory for a plasma: II. Reciprocal relations," Physica A, vol. 89, pp. 225-244, 1977.

[33] H. Van Erkelens and W. A. Van Leeuwen, "RelativisticBoltzmann theory for a plasma: III. Viscous phenomena," Physica A, vol. 90, pp. 97-108, 1978.

[34] I. S. Gradshteyn and I. M. Ryzhik, Table of Integrals, Series, and Products, Edited by: A. Jeffrey, D. Zwillinger, Academic Press, 7 th edition, 2007.

[35] K. Tuchin, "On viscous flow and azimuthal anisotropy of the quark-gluon plasma in a strong magnetic field," Journal of Physics G, vol. 39, no. 2, Article ID 025010, 2012.

[36] L. V. Gribov, E. M. Levin, and M. G. Ryskin, "Semihard processes in QCD," Physics Reports, vol. 100, no. 1-2, pp. 1-150, 1983.

[37] J. P. Blaizot and A. H. Mueller, "The early stage of ultrarelativistic heavy ion collisions," Nuclear Physics B, vol. 289, pp. 847-860, 1987.

[38] H. Song and U. W. Heinz, "Suppression of elliptic flow in a minimally viscous quark-gluon plasma," Nuclear Physics B, vol. 658, pp. 279-283, 2008.
[39] P. Romatschke and U. Romatschke, "Viscosity information from relativistic nuclear collisions: how perfect is the fluid observed at RHIC?” Physical Review Letters, vol. 99, no. 17, Article ID 172301, 4 pages, 2007.

[40] K. Dusling and D. Teaney, "Simulating elliptic flow with viscous hydrodynamics," Physical Review C, vol. 77, Article ID 034905, 19 pages, 2008.

[41] E. S. Weibel, "Spontaneously growing transverse waves in a plasma due to an anisotropic velocity distribution," Physical Review Letters, vol. 2, pp. 83-84, 1959.

[42] S. Mrowczynski, "Color collective effects at the early stage of ultrarelativistic heavy-ion collisions," Physical Review C, vol. 49, pp. 2191-2197, 1994.

[43] P. B. Arnold, G. D. Moore, and L. G. Yaffe, "Transport coefficients in high temperature gauge theories, 2. Beyond leading log," Journal of High Energy Physics, vol. 305, p. 51, 2003.

[44] G. Baym, H. Monien, C. J. Pethick, and D. G. Ravenhall, "Transverse interactions and transport in relativistic quarkgluon and electromagnetic plasmas," Physical Review Letters, vol. 64, no. 16, pp. 1867-1870, 1990.

[45] A. I. Nikishov and V. I. Ritus, "Quantum processes in the field of a plane electromagnetic wave and in a constant field. I," Soviet Physics, vol. 19, pp. 529-541, 1964.

[46] A. A. Sokolov and I. M. Ternov, "On polarization and spin effects in synchrotron radiation theory," Soviet PhysicsDoklady, vol. 8, p. 1203, 1964, Doklady Akademii Nauk SSSR, vol. 153, pp. 1052, 1964, PHDOE, vol. 8, pp. 1203-1205, 1964.

[47] V. I. Ritus, "Quantum effects of the interaction of elementary particles with an intense electromagnetic field," Journal of Soviet Laser Research, vol. 6, no. 5, pp. 497-617, 1985.

[48] V. B. Berestetsky, E. M. Lifshitz, and L. P. Pitaevsky, Quantum Electrodynamics, vol. 4 of Course Of Theoretical Physics, Pergamon, Oxford, UK, 1982.

[49] J. D. Jackson, "On understanding spin-flip synchrotron radiation and the transverse polarization of electrons in storage rings," Reviews of Modern Physics, vol. 48, pp. 417-433, 1976.

[50] M. Gyulassy and X. N. Wang, "Multiple collisions and induced gluon bremsstrahlung in QCD," Nuclear Physics B, vol. 420, no. 3, pp. 583-614, 1994.

[51] R. Baier, Y. L. Dokshitzer, S. Peigne, and D. Schi, "Induced gluon radiation in a QCD medium," Physics Letters B, vol. 345, pp. 277-286, 1995.

[52] E. V. Shuryak and I. Zahed, "Jet Quenching in high energy heavy ion collisions by QCD synchrotron-like radiation," Physical Review D, vol. 67, Article ID 054025, 2003.

[53] D. E. Kharzeev, "Universal upper bound on the energy of a parton escaping from the strongly coupled quark-gluon matter," In press, http://arxiv.org/abs/0806.0358.

[54] B. G. Zakharov, "Parton energy loss due to synchrotron-like gluon emission," JETP Letters, vol. 88, no. 8, pp. 475-480, 2008.

[55] K. Tuchin, "Photon decay in a strong magnetic field in heavyion collisions," Physical Review C, vol. 83, no. 1, Article ID 017901, 3 pages, 2011.

[56] V. N. Baier and V. M. Katkov, "Quantum effects in magnetic bremsstrahlung," Physics Letters A, vol. 25, no. 7, pp. 492-493, 1967.

[57] K. Marasinghe and K. Tuchin, "Quarkonium dissociation in quark-gluon plasma via ionization in a magnetic field," Physical Review C, vol. 84, no. 4, Article ID 044908, 8 pages, 2011.

[58] K. Tuchin, "J/ $\psi$ dissociation in parity-odd bubbles," Physics Letters B, vol. 705, pp. 482-486, 2011. 
[59] L. D. Landau and L. E. Lifshitz, Quantum Mechanics NonRelativistic Theory, Butterworth-Heinemann, 3rd edition.

[60] B. Machet and M. I. Vysotsky, "Modification of Coulomb law and energy levels of the hydrogen atom in a superstrong magnetic field," Physical Review D, vol. 83, no. 2, Article ID 025022, 12 pages, 2011.

[61] V. S. Popov, B. M. Karnakov, and V. D. Mur, "Quasiclassical theory of atomic ionization in electric and magnetic fields," Physics Letters A, vol. 229, no. 5, pp. 306-312, 1997.

[62] V. S. Popov, B. M. Karnakov, and V. D. Mur, "Relativistic version of the imaginary time method," Physics Letters A, vol. 250, pp. 20-24, 1998.

[63] V. S. Popov, B. M. Karnakov, and V. D. Mur, "Ionization of atoms in electric and magnetic fields and the imaginary time method," Journal of Experimental and Theoretical Physics, vol. 86, no. 5, pp. 860-874, 1998.

[64] L. V. Keldysh, "Diagram technique for nonequilibrium processes," Soviet Physics, vol. 20, pp. 1018-1026, 1965.

[65] L. D. Landau and L. E. Lifshitz, Quantum Mechanics NonRelativistic Theory, Butterworth-Heinemann, 3rd edition.

[66] V. S. Popov, "Tunnel and multiphoton ionization of atoms and ions in a strong laser field (Keldysh theory)," Physics-Uspekhi, vol. 47, no. 9, pp. 855-885, 2004.

[67] D. Kharzeev, "Parity violation in hot QCD: why it can happen, and how to look for it," Physics Letters B, vol. 633, pp. 260-264, 2006.

[68] D. Kharzeev and A. Zhitnitsky, "Charge separation induced by P-odd bubbles in QCD matter," Nuclear Physics A, vol. 797, no. 1-2, pp. 67-79, 2007.

[69] K. Fukushima, D. E. Kharzeev, and H. J. Warringa, "Chiral magnetic effect," Physical Review D, vol. 78, no. 7, Article ID 074033, 14 pages, 2008.

[70] D. E. Kharzeev, “Topologically induced local $P$ and $C P$ violation in QCD × QED," Annals of Physics, vol. 325, pp. 205-218, 2010.

[71] G. Basar, G. V. Dunne, and D. E. Kharzeev, "Chiral magnetic spirals," Physical Review Letters, vol. 104, no. 23, Article ID 232301, 4 pages, 2010.

[72] M. Asakawa, A. Majumder, and B. Muller, "Electric charge separation in strong transient magnetic fields," In press, http://arxiv.org/abs/1003.2436.

[73] B. I. Abelev, M. M. Aggarwal, Z. Ahammed et al., "Azimuthal charged-particle correlations and possible local strong parity violation," Physical Review Letters, vol. 103, no. 25, Article ID 251601, 7 pages, 2009.

[74] B. I. Abelev, M. M. Aggarwal, Z. Ahammed et al., "Observation of charge-dependent azimuthal correlations and possible local strong parity violation in heavy ion collisions," Physical Review C, vol. 81, Article ID 054908, 2010.

[75] N. N. Ajitanand, R. A. Lacey, A. Taranenko, and J. M. Alexander, "New method for the experimental study of topological effects in the quark-gluon plasma," Physical Review C, vol. 83, no. 1, 5 pages, 2011.

[76] A. M. Perelomov, V. S. Popov, and M. V. Terentev, ZhurnalEksperimental'noi i Teoreticheskoi Fiziki, vol. 50, p. 1393, 1966.

[77] A. M. Perelomov, V. S. Popov, and M. V. Terentev, Zhurnal Eksperimental'noi iTeoreticheskoi Fiziki, vol. 51, p. 309, 1966.

[78] V. S. Popov, V. P. Kuznetzov, and A. M. Perelomov, Zhurnal Eksperimental'noi i Teoreticheskoi Fiziki, vol. 53, p. 331, 1967.

[79] G. D. Moore and M. Tassler, "The sphaleron rate in SU(N) gauge theory," Journal of High Energy Physics, vol. 1102, article 105, 2011.
[80] T. Matsui and H. Satz, "J/ $\psi$ suppression by quark-gluon plasma formation," Physics Letters B, vol. 178, no. 4, pp. 416-422, 1986.

[81] A. A. Sokolov and I. M. Ternov, Synchrotron Radiation, Pergamon Press, Oxford, UK, 1968.

[82] M. G. Baring, "Synchrotron emission, pair production and annihilation in strongly magnetized relativistic plasmas," Monthly Notices of the Royal Astronomical Society, vol. 235, pp. 51-78, 1988.

[83] H. Herold, H. Ruder, and G. Wunner, "Cyclotron emission in strongly magnetized plasmas," Astronomy \& Astrophysics, vol. 115, no. 1, pp. 90-96, 1982.

[84] A. K. Harding and R. Preece, "Quantized synchrotron radiation in strong magnetic fields," Astrophysical Journal, vol. 319, pp. 939-950, 1987.

[85] H. G. Latal, "Cyclotron radiation in strong magnetic fields," Astrophysical Journal, vol. 309, pp. 372-382, 1986.

[86] A. K. Harding, "One-photon pair annihilation in magnetized relativistic plasmas," Astrophysical Journal, vol. 300, pp. 167-177, 1986.

[87] G. Wunner, J. Paez, H. Herold, and H. Ruder, "One-quantum annihilation of polarized electron-positron pairs in strong magnetic fields," Astronomy \& Astrophysics, vol. 170, no. 1, pp. 179-186, 1986.

[88] G. Wunner, "Comparison of $1 \gamma$ and $2 \gamma$ pair annihilation in strong magnetic fields," Physical Review Letters, vol. 42, pp. 7982, 1979.

[89] A. J. Mizher, M. N. Chernodub, and E. S. Fraga, "Phase diagram of hot QCD in an external magnetic field: Possible splitting of deconfinement and chiral transitions," Physical Review D, vol. 82, no. 10, Article ID 105016, 16 pages, 2010.

[90] E. S. Fraga and A. J. Mizher, "Can a strong magnetic background modify the nature of the chiral transition in QCD?" Nuclear Physics A, vol. 820, pp. 103c-106c, 2009.

[91] E. S. Fraga and L. F. Palhares, "Deconfinement in the presence of a strong magnetic background: an exercise within the MIT bag model," Physical Review D, vol. 86, Article ID 016008, 7 pages, 2012.

[92] R. Gatto and M. Ruggieri, "Deconfinement and chiral symmetry restoration in a strong magnetic background," Physical Review D, vol. 83, no. 3, Article ID 034016, 12 pages, 2011.

[93] R. Gatto and M. Ruggieri, "Dressed Polyakov loop and phase diagram of hot quark matter in a magnetic field," Physical Review D, vol. 82, no. 5, Article ID 054027, 10 pages, 2010.

[94] A. A. Osipov, B. Hiller, A. H. Blin, and J. da Providencia, "Dynamical chiral symmetry breaking by a magnetic field and multi-quark interactions," Physics Letters B, vol. 650, pp. 262267, 2007.

[95] K. Kashiwa, "Entanglement between chiral and deconfinement transitions under strong uniform magnetic background field," Physical Review D, vol. 83, no. 11, Article ID 117901, 4 pages, 2011.

[96] C. V. Johnson and A. Kundu, "External fields and chiral symmetry breaking in the Sakai-Sugimoto model," Journal of High Energy Physics, vol. 2008, no. 12, p. 53, 2008.

[97] S. Kanemura, H. T. Sato, and H. Tochimura, "Thermodynamic Gross-Neveu model in a constant electromagnetic field," Nuclear Physics B, vol. 517, no. 1-3, pp. 567-598, 1998.

[98] J. Alexandre, K. Farakos, and G. Koutsoumbas, "Magnetic catalysis in three-dimensional QED at finite temperature: beyond the constant mass approximation," Physical Review D, vol. 63, no. 6, Article ID 065015, 13 pages, 2001. 
[99] N. O. Agasian and S. M. Fedorov, "Quark-hadron phase transition in a magnetic field," Physics Letters B, vol. 663, pp. 445-449, 2008.

[100] F. Preis, A. Rebhan, and A. Schmitt, "Inverse magnetic catalysis in dense holographic matter," Journal of High Energy Physics, vol. 2011, article 33, 2011.

[101] V. P. Gusynin, V. A. Miransky, and I. A. Shovkovy, "Dimensional reduction and catalysis of dynamical symmetry breaking by a magnetic field," Nuclear Physics B, vol. 462, no. 2-3, pp. 249-290, 1996.

[102] V. A. Miransky and I. A. Shovkovy, "Magnetic catalysis and anisotropic confinement in QCD," Physical Review D, vol. 66, no. 4, Article ID 045006, 9 pages, 2002.

[103] M. N. Chernodub, "Superconductivity of QCD vacuum in strong magnetic field," Physical Review D, vol. 82, no. 8, Article ID 085011, 17 pages, 2010.

[104] Y. Hidaka and A. Yamamoto, "Charged vector mesons in a strong magnetic field," In press, http://arxiv.org/abs/1209.0007.

[105] M. N. Chernodub, "Vafa-Witten theorem, vector meson condensates, and magnetic-field-induced electromagnetic superconductivity of vacuum," Physical Review D, vol. 86, no. 10, Article ID 107703, 2 pages, 2012.

[106] J. K. Boomsma and D. Boer, "Influence of strong magnetic fields and instantons on the phase structure of the two-flavor Nambu-Jona-Lasinio model," Physical Review D, vol. 81, Article ID 074005, 9 pages, 2010.

[107] I. A. Shushpanov and A. V. Smilga, "Quark condensate in a magnetic field," Physics Letters B, vol. 402, no. 3-4, pp. 351-358, 1997.

[108] T. D. Cohen, D. A. McGady, and E. S. Werbos, "Chiral condensate in a constant electromagnetic field," Physical Review C, vol. 76, no. 5, Article ID 055201, 9 pages, 2007.

[109] N. O. Agasian, "Chiral thermodynamics in a magnetic field," Physics of Atomic Nuclei, vol. 64, pp. 554-560, 2001, Yadernaya Fizika, vol. 64, pp. 608, 2001.

[110] B. V. Galilo and S. N. Nedelko, "Impact of the strong electromagnetic field on the QCD effective potential for homogeneous Abelian gluon field configurations," Physical Review D, vol. 84, no. 9, Article ID 094017, 8 pages, 2011.

[111] M. D’Elia, S. Mukherjee, and F. Sanfilippo, "QCD phase transition in a strong magnetic background," Physical Review D, vol. 82, no. 5, Article ID 051501, 5 pages, 2010.

[112] P. Cea and L. Cosmai, "Color dynamics in external fields," Journal of High Energy Physics, vol. 508, p. 79, 2005.

[113] P. Cea and L. Cosmai, "Abelian chromomagnetic fields and confinement," Journal of High Energy Physics, vol. 302, p. 31, 2003.

[114] P. Cea, L. Cosmai, and M. D’Elia, “QCD dynamics in a constant chromomagnetic field," Journal of High Energy Physics, vol. 712, p. $97,2007$.

[115] G. S. Bali, F. Bruckmann, G. Endrődi et al., "The QCD phase diagram for external magnetic fields," Journal of High Energy Physics, vol. 2012, article 44, 2012.

[116] G. S. Bali, F. Bruckmann, G. Endrodi et al., "The finite temperature QCD transition in external magnetic fields," In press, http://arxiv.org/abs/1111.5155.

[117] G. S. Bali, F. Bruckmann, G. Endrodi, Z. Fodor, S. D. Katz, and A. Schafer, "QCD quark condensate in external magnetic fields," Physical Review D, vol. 86, no. 7, Article ID 071502, 6 pages, 2012.

[118] E.-M. Ilgenfritz, M. Kalinowski, M. Muller-Preussker, B. Petersson, and A. Schreiber, "Two-color QCD with staggered fermions at finite temperature under the influence of a magnetic field," Physical Review D, vol. 85, no. 11, Article ID 114504, 12 pages, 2012.

[119] Y. Aoki, G. Endrodi, Z. Fodor, S. D. Katz, and K. K. Szabó, “The order of the quantum chromodynamics transition predicted by the standard model of particle physics," Nature, vol. 443, no. 7112, pp. 675-678, 2006.

[120] S.-I. Nam and C.-W. Kao, "Chiral restoration at finite T under the magnetic field with the meson-loop corrections," Physical Review D, vol. 83, no. 9, Article ID 096009, 14 pages, 2011.

[121] M. D'Elia and F. Negro, "Chiral properties of strong interactions in a magnetic background," Physical Review D, vol. 83, Article ID 114028, 2011.

[122] P. V. Buividovich, M. N. Chernodub, D. E. Kharzeev, T. Kalaydzhyan, E. V. Luschevskaya, and M. I. Polikarpov, "Magnetic-field-induced insulator-conductor transition in SU(2) quenched lattice gauge theory," Physical Review Letters, vol. 105, no. 13, Article ID 132001, 2010.

[123] F. Bruckmann and G. Endrodi, "Dressed Wilson loops as dual condensates in response to magnetic and electric fields," Physical Review D, vol. 84, Article ID 074506, 2011. 

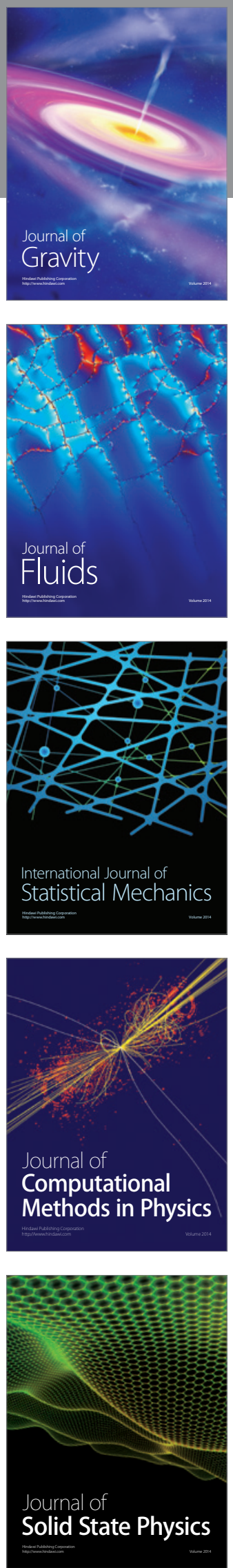

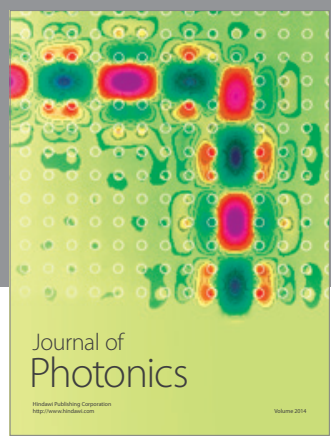

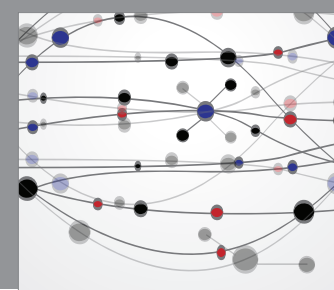

The Scientific World Journal

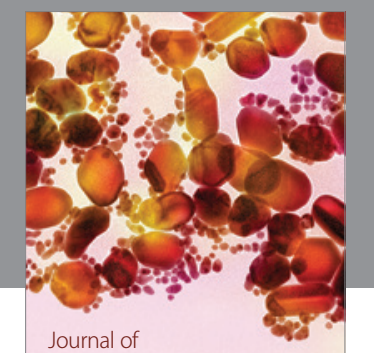

Soft Matter
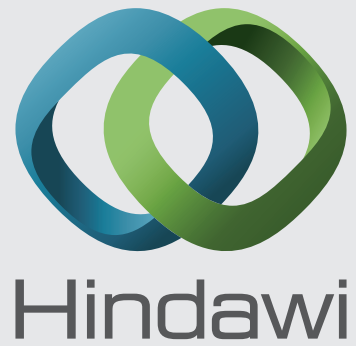

Submit your manuscripts at

http://www.hindawi.com
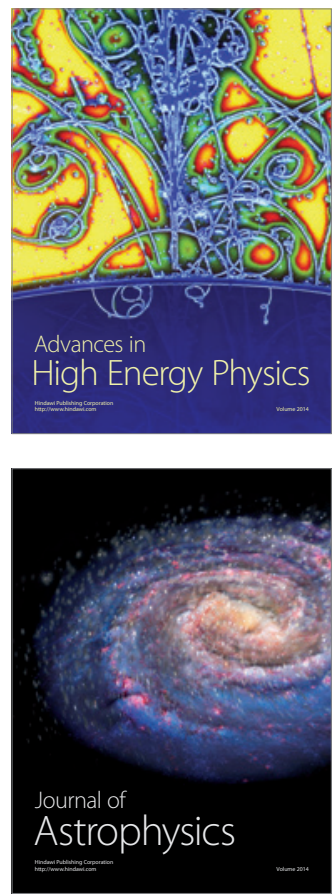
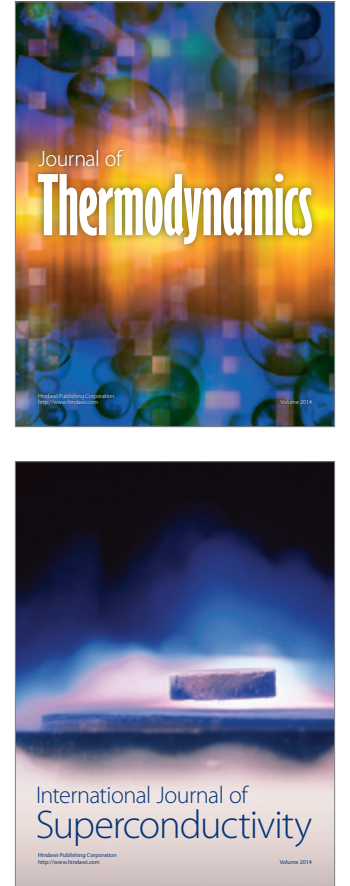
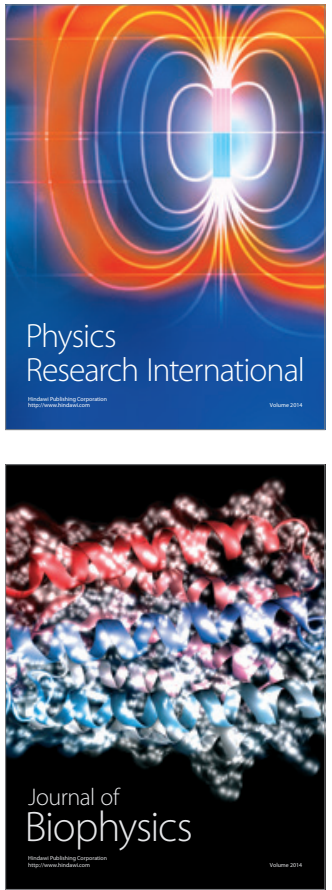
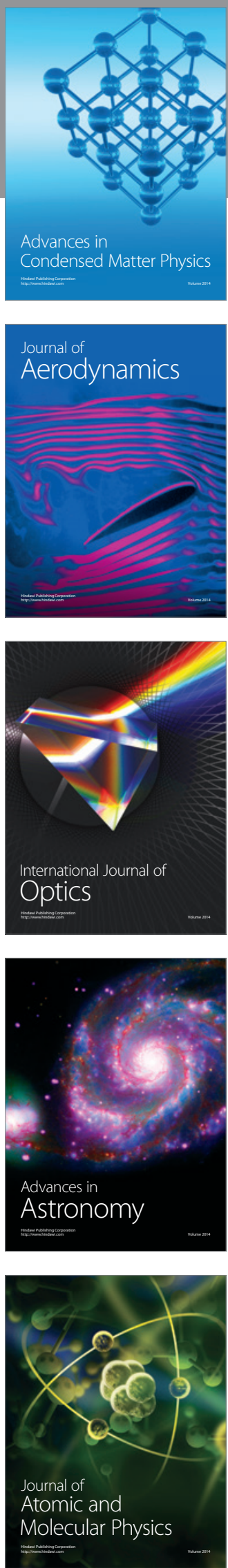